\title{
A biopsychosocial approach to fatigue in patients on chronic haemodialysis treatment
}

Citation for published version (APA):

Brys, A. (2021). A biopsychosocial approach to fatigue in patients on chronic haemodialysis treatment. [Doctoral Thesis, Maastricht University]. Gildeprint Drukkerijen. https://doi.org/10.26481/dis.20210326ab

Document status and date:

Published: 01/01/2021

DOI:

10.26481/dis.20210326ab

Document Version:

Publisher's PDF, also known as Version of record

\section{Please check the document version of this publication:}

- A submitted manuscript is the version of the article upon submission and before peer-review. There can be important differences between the submitted version and the official published version of record.

People interested in the research are advised to contact the author for the final version of the publication, or visit the DOI to the publisher's website.

- The final author version and the galley proof are versions of the publication after peer review.

- The final published version features the final layout of the paper including the volume, issue and page numbers.

Link to publication

\footnotetext{
General rights rights.

- You may freely distribute the URL identifying the publication in the public portal. please follow below link for the End User Agreement:

www.umlib.nl/taverne-license

Take down policy

If you believe that this document breaches copyright please contact us at:

repository@maastrichtuniversity.nl

providing details and we will investigate your claim.
}

Copyright and moral rights for the publications made accessible in the public portal are retained by the authors and/or other copyright owners and it is a condition of accessing publications that users recognise and abide by the legal requirements associated with these

- Users may download and print one copy of any publication from the public portal for the purpose of private study or research.

- You may not further distribute the material or use it for any profit-making activity or commercial gain

If the publication is distributed under the terms of Article $25 \mathrm{fa}$ of the Dutch Copyright Act, indicated by the "Taverne" license above, 


\section{A biopsychosocial approach to fatigue in}

patients on chronic haemodialysis treatment 


\section{Colofon}

(C) Copyright Astrid Brys, Maastricht, 2021

All rights reserved. No part of this book may be reproduced or transmitted in any form

or by any means, without prior permission in writing by the author, or when appropriate, by the publishers of the publications.

ISBN 9789464191417

Cover design: Liesbeth Mariën

Layout: Tiny Wouters

Printed by: Gildeprint - the Netherlands 


\title{
A biopsychosocial approach to fatigue in patients on chronic haemodialysis treatment
}

\author{
DISSERTATION \\ to obtain the degree of doctor at \\ Maastricht University under the authority \\ of Rector Magnificus Prof. Dr. Rianne M. Letschert
}

\&

Catholic University of the Sacred Heart, Italy

under the authority of Rector Magnificus Prof. Dr. Franco Anelli

in accordance with the decision of the Board of Deans,

to be defended in public

on Friday March 26, 2021 at 12:00 P.M. in Maastricht

and

on Thursday April 8, 2021 at 11:00 A.M. in Rome

by

Astrid Denise Huguette Maria Brys 


\section{Supervisors}

Prof. dr. G. Gambaro (Catholic University of the Sacred Heart, Rome, Italy)

Prof. dr. C. M. van Heugten (Maastricht University, Maastricht, the Netherlands)

\section{Co-supervisors}

Dr. M. Bossola (Catholic University of the Sacred Heart, Rome, Italy)

Dr. B. Lenaert (Maastricht University, Maastricht, the Netherlands)

\section{Italian co-ordinator}

Prof. dr. O. Parolini (Catholic University of the Sacred Heart, Rome, Italy)

\section{Assessment committee Maastricht}

Prof. dr. R.W.H.M. Ponds (chair) (Maastricht University, Maastricht)

Dr. C.J.A.M. Konings (Catharina Hospital, Eindhoven)

Prof. dr. C.A.M. van Bennekom (University of Amsterdam, Amsterdam)

Prof. dr. G. Zuccalà (Catholic University of the Sacred Heart, Rome)

\section{Assessment committee Rome}

Prof. dr. O. Parolini (Catholic University of the Sacred Heart, Rome, Italy)

Prof. dr. G. Gambaro (Catholic University of the Sacred Heart, Rome, Italy)

Prof. dr. C. M. van Heugten (Maastricht University, Maastricht, the Netherlands)

Additional members will be assigned. 
'Per aspera ad astra' 


\section{Contents}

Chapter $1 \quad$ General introduction

Part I The role of inflammation in fatigue symptoms in chronic haemodialysis patients

Chapter 2 Serum interleukin-6 and endotoxin levels and their relationship

with fatigue and depressive symptoms in patients on

chronic haemodialysis

Chapter 3 Peridialytic serum cytokine levels and their relationship

with postdialysis fatigue and recovery in patients on chronic haemodialysis - A preliminary study

Part II The relationship of psychological and contextual variables with fatigue in chronic haemodialysis patients

Chapter 4 Exploring the diurnal course of fatigue in patients on

haemodialysis treatment and its relation with depressive symptoms and classical conditioning

Chapter 5 Daily physical activity in patients on chronic haemodialysis and its relation to fatigue and depressive symptoms

Part III The added value of the Experience Sampling Methodology 107 in assessing fatigue in chronic haemodialysis patients

Chapter $6 \quad$ mHealth based Experience Sampling Method to identify fatigue in the context of daily life in haemodialysis patients

Chapter $7 \quad$ Unravelling fatigue in haemodialysis patients: comparing retrospective reports to real-time assessments with an mHealth Experience Sampling Method 
Chapter $8 \quad$ General discussion

151

Addendum Summary

171

Samenvatting

179

Sintesi

187

Impact paragraph

195

List of publications

203

Words of gratitude

209

Curriculum Vitae

215 



\section{Chapter 1}

General introduction 



\section{Background}

\section{Chronic kidney disease and haemodialysis treatment}

Chronic kidney disease (CKD) refers to a gradual and irreversible loss of kidney function. CKD arises from heterogeneous disease pathways, but diabetes and hypertension are globally the main causes of CKD. ${ }^{1}$ When end-stage kidney disease is reached, renal replacement therapy will be required to sustain life over the long term. ${ }^{2}$ Worldwide, the prevalence of CKD is estimated at $13.4 \%^{3}$ and it is predicted that CKD will be among the 15 leading causes of death by $2030 .{ }^{4}$ Approximately three million people receive renal replacement therapy because of end-stage kidney disease. ${ }^{5}$ Haemodialysis (HD) is by far the most common form of renal replacement therapy with an estimated prevalence of 465 and 1437 per million population in Europe and the U.S., respectively. ${ }^{6-8}$ During the HD treatment, the circulating blood is put through a medical apparatus outside the body (i.e. the dialysis machine) equipped with a semi-permeable membrane. This artificial kidney filters the blood, removes waste products and excessive fluid, and corrects electrolyte imbalances. The purified blood is then returned into the body. A typical HD patient's treatment schedule consists of a thrice weekly HD session lasting 3-4 hours each time.

\section{Symptom burden and quality of life in chronic HD patients}

Patients on chronic HD treatment experience a substantial symptom burden due to the metabolic derangements and psychosocial impact associated with CKD and the HD therapy. The most frequently reported symptoms include fatigue or a lack of energy, a dry skin and itching, bone or joint pain, a dry mouth, muscle cramps, dizziness, decreased appetite, sleep disturbances and depressive feelings. ${ }^{9,10}$ HD patients' symptom burden is comparable to that of palliative cancer patients and greatly impacts on their health-related quality of life. ${ }^{9}$ While palliative care treatment is dominated by symptom management and comfort therapy, relatively little attention is paid to symptom burden in routine clinical practice in HD patients, as treatment is mainly focused on minimizing morbidity and mortality. ${ }^{10}$ Given that HD treatment is usually received for many years, symptom relief merits equal attention in the management of HD patients. In order to improve HD patients' health-related quality of life, research into the development and treatment of these symptoms is warranted. 
This thesis will focus on fatigue, the most frequently reported complaint by HD patients.

\section{Fatigue in HD patients}

The prevalence of HD patients suffering from fatigue ranges from $42-89 \%$, depending on the measurement method used to assess fatigue. ${ }^{11}$ Moreover, fatigue is one of the most prioritized outcomes by HD patients. ${ }^{12}$ The Standardized Outcomes in Nephrology (SONG-HD) initiative, establishing shared priorities of patients, caregivers, clinicians, researchers, policy makers, and relevant stakeholders, has identified fatigue as one of the core outcome measures to report across trials in haemodialysis. ${ }^{13}$

\section{Definition and qualities}

Due to its inherently subjective nature, fatigue is difficult to determine. Fatigue has been defined as "an overwhelming, debilitating, and sustained sense of exhaustion that is likely to decrease one's ability to carry out daily activities, including the ability to work effectively and to function at one's usual level in family or social roles". ${ }^{14}$ Moreover, different qualities of fatigue may be distinguished. For instance, fatigue can manifest at a physical and/or mental level. ${ }^{15,16}$ The former is characterized by sensations such as muscle weakness, a lack of energy or sleepiness. The latter may be experienced as apathy, the inability to concentrate, or decreased motivation. Alternatively, fatigue may be categorised as central versus peripheral. ${ }^{17}$ Central fatigue arises from the central nervous system (CNS) and may manifest as absence of motivation and failure to initiate or sustain a task (mentally or physically), whereas peripheral fatigue occurs at the muscular level and may manifest as loss of muscle strength or performance decrements. Further, besides a general sense of fatigue, patients receiving HD treatment may experience a more acute and intermittent fatigue that particularly occurs after the HD session and may promote resting or sleeping to allow recovery from HD treatment. This phenomenon is conventionally termed postdialysis fatigue (PDF), and occurs in $20-80 \%$ of chronic HD patients. ${ }^{18}$ Patients typically describe this symptom as feeling drained, worn out or exhausted as a result of the HD treatment. ${ }^{18}$ However, using dualistic distinctions to describe fatigue components (e.g. physical vs. mental, central vs. peripheral, general vs. PDF) risks ignoring the multidimensional nature of which fatigue may consist. ${ }^{19}$ 


\section{Correlates and aetiology}

Despite its high prevalence and impact on quality of life, the aetiology of fatigue in HD patients remains largely elusive. Previous research has attempted to identify potential contributing factors to fatigue in HD patients and has investigated its relationship with sociodemographic, HD treatment-related, clinical and laboratory variables. However, findings are often conflicting and inconsistent.

\section{Sociodemographic factors}

A gender association, suggesting female HD patients experience more fatigue than males, has been found in some studies but not in others. ${ }^{11,20}$ With regard to ethnicity, some studies report that non-white race patients experience less fatigue, whereas other studies do not find differences between ethnic or racial groups. ${ }^{11,20}$ Higher age is associated with higher fatigue severity in HD patients, ${ }^{20}$ although there are discrepant findings as well. ${ }^{21}$ The link between fatigue and level of education, employment or marital status is unclear. ${ }^{20,22}$

\section{HD Treatment-related and clinical factors}

Similarly, findings with respect to HD treatment-related and clinical variables are not univocal. ${ }^{11}$ For instance, with respect to dialysis vintage, some studies suggest that fatigue increases as a function of the number of years on dialysis, ${ }^{23}$ whereas other studies show the opposite, with longer dialysis vintage predicting less fatigue. ${ }^{24,25}$ Further, some reports did not find a significant association between fatigue and parameters of nutritional status (e.g. body mass index or serum (pre-) albumin levels), ${ }^{26-29}$ whereas more recent reports found that fatigue severity correlated with hypoalbuminemia and anorexia. ${ }^{17,23,30}$ Associations between fatigue and comorbidities, sleep disorders and physical inactivity, have been reported more consistently. Indeed, fatigue positively correlates with the number of coexisting chronic illnesses, ${ }^{17,23,31}$ poor sleep quality due to insomnia, restless legs or sleep apnea, ${ }^{17,26}$ and physical inactivity, ${ }^{17,32-34}$ which may represent a consequence as well as a contributing factor to fatigue in HD patients. ${ }^{35}$ Despite inadequate dialysis dose has been suspected as a source of fatigue, the few studies that investigated the relationship between haemodialysis adequacy $(K t / V)$ and fatigue, did not find a significant association. ${ }^{22,23,28,36}$ However, this may be explained by the relative homogeneity of $K t / V$-values in the studied HD populations. ${ }^{11}$ 


\section{Biological factors}

Among commonly assessed laboratory parameters are: haemoglobin ( $\mathrm{Hb}$ ) levels, serum creatinine and blood urea nitrogen concentration, parathyroid hormone (PTH) levels, serum calcium and phosphorus levels, and serum albumin levels. Anaemia, a consequence of impaired kidney function, may cause fatigue. ${ }^{1}$ However, several studies did not find a significant correlation between $\mathrm{Hb}$ levels and fatigue in HD patients. ${ }^{20,23}$ This may be attributed to many HD patients receiving therapy with erythropoietin stimulating agents (ESA) in order to achieve relatively homogenous $\mathrm{Hb}$ levels within the range of 11.0 to $12.0 \mathrm{~g} / \mathrm{dl}$, as recommended by the Kidney Disease Outcomes Quality Initiative (KDOQI) guidelines. ${ }^{2}$ As such, other factors than anaemia could account for fatigue in HD patients. Indeed, fatigue in HD patients might be related to uraemia or hyperparathyroidism secondary to kidney failure. However, neither urea levels or PTH levels, nor serum creatinine levels or calcium and phosphorus levels have been consistently demonstrated to correlate with fatigue in HD patients. ${ }^{22,23,25,26,36}$ Again, the absence of an association may result from the relative homogeneity of these variables' values, maintained within recommended ranges for HD patients.

Intriguingly, there has been increasing interest over the last years in the idea of chronic inflammation triggering fatigue in chronic HD patients. ${ }^{17}$ In other illnesses, such as infectious diseases, ${ }^{37}$ auto-immune disorders (e.g. rheumatoid arthritis, ${ }^{38,39}$ psoriasis ${ }^{40}$ ), neurodegenerative disorders (e.g. multiple sclerosis $(\mathrm{MS})^{41,42}$ ), cancer, ${ }^{43-45}$ the geriatric frailty syndrome, ${ }^{46}$ but also mood disorders ${ }^{47}$ and chronic fatigue syndrome (CFS), ${ }^{48}$ there is growing evidence for the involvement of inflammation in the development of fatigue. Pro-inflammatory cytokines, such as tumour necrosis factor alpha (TNF- $\alpha$ ), interleukin 1 beta (IL-1 ) and interleukin 6 (IL-6), may interact with the CNS through immune-to-brain communication pathways. ${ }^{49}$ As such, they may provoke a constellation of non-specific illness symptoms, often referred to as 'sickness behaviour', which may include fatigue, decreased physical activity, reduced ability to concentrate, altered mood state, withdrawal from social interaction and loss of appetite. ${ }^{50,51}$ It may be seen as a biologically triggered coping mechanism by inducing rest in order to conserve energy and thus allow recovery to take place. ${ }^{52}$ Moreover, it has been suggested that pro-inflammatory cytokines may mediate fatigue indirectly by inducing sleep disorders, depression or anxiety. ${ }^{17}$ Finally, they may also contribute to fatigue by acting peripherally. For instance, it has been hypothesized that pro- 
inflammatory cytokines may enhance activity of muscular ergoreceptors, which sense muscle contraction and relaxation. ${ }^{17,53}$ Furthermore, pro-inflammatory cytokines may contribute to protein catabolism, hypoalbuminemia, malnutrition, or erythropoesis suppression, which have been associated with fatigue. ${ }^{17}$ In contrast, antiinflammatory cytokines, such as interleukin 10 (IL-10), inhibit the production and attenuate the signalling of pro-inflammatory cytokines. ${ }^{47}$ Therefore, they may regulate the intensity and duration of the pro-inflammatory cytokine induced symptomatology. ${ }^{47}$ In the HD population, up to half of the chronic HD patients shows evidence of a chronic inflammatory state. ${ }^{54}$ This may be attributed to both end-stage kidney disease and dialysis-related factors. Indeed, renal failure itself results in the accumulation of pro-inflammatory components, the reduction of plasma antioxidant activity and increased oxidative stress. ${ }^{55,56}$ Moreover, the uremic environment may disrupt the intestinal barrier, facilitating translocation of gut microbiota. ${ }^{57}$ Furthermore, impaired humoral and cellular immunity, vascular accesses or cooccurring diabetes mellitus, make HD patients more susceptible to infections. ${ }^{58}$ In addition, the dialysis procedure itself may stimulate cytokine production by circulating mononuclear cells, which may be activated when the individuals' blood interacts with bioincompatible dialysis membranes. ${ }^{55,56}$ Finally, microbial or endotoxin contaminated dialysis water, may further induce transmembrane activation of circulating immune cells. $^{55,56}$ The hypothesis that fatigue in HD patients may be induced by proinflammatory cytokines is an interesting avenue of investigation, which will be further explored in the first part of this thesis.

\section{Psychosocial and contextual factors}

However, fatigue in HD patients is likely explained by other than biological factors as well. Psychological, social and contextual variables may have a direct impact on individuals' fatigue experience or may interact with biological variables in their impact on fatigue. For instance, in the general population, fatigue and depression are closely interrelated and depression may manifest itself, among other symptoms, as tiredness or a lack of energy. ${ }^{59}$ Interestingly, the development of depression has also been associated with increased pro-inflammatory cytokine levels. ${ }^{47,60}$ In HD patients, depression is present in a third of the HD population ${ }^{61}$ and previous studies already demonstrated an association between depressive symptoms and fatigue in HD patients. ${ }^{11,17}$ Furthermore, the involvement of psychological factors in the aetiology of fatigue in other chronic conditions, such as CFS and MS, has already been 
investigated. ${ }^{62,63}$ Aetiological models that incorporate both physiological and psychological factors have been formulated to clarify the development and maintenance of fatigue in CFS and MS. ${ }^{62,63}$ Herein, biological and psychological factors that may contribute to fatigue are integrated, rather than separated, as they may influence each other and elicit a vicious cycle of fatigue symptoms. For instance, biological factors, such as inflammation, may initially trigger fatigue, but individuals' emotional, behavioural or cognitive response may consolidate or aggravate the experience of fatigue. ${ }^{62,63}$ A similar approach to fatigue in HD patients may be interesting to unravel and advance the understanding of this complex symptom. To what extent individual psychological (e.g. depressive feelings), social (e.g. social company) and contextual (e.g. daily activity and location) factors may be related to fatigue experience in HD patients, will be further explored in the second and third part of this thesis.

\section{Fatigue assessment}

There is currently no universally accepted or validated fatigue scale specifically developed for the HD population which takes into account the different psychological, social, behavioural and contextual variables that may contribute to fatigue. In fact, a recently published systematic review on fatigue measurement instruments applied in HD patients found that 43 different instruments were used across 123 studies. $^{64}$ Among these fatigue instruments, the Fatigue Severity Scale (FSS), Short Form-36 vitality subscale (SF-36 VS), Kidney Disease Quality of Life-Short Form (KDQOL-SF) and Visual Analogue Scale (VAS) are the most commonly administered questionnaires to assess fatigue in HD patients. ${ }^{64}$ However, these conventional instruments have several limitations. First, the majority of these instruments assess fatigue unidimensionally, usually focussing on fatigue severity in general. ${ }^{65}$ Second, they use (different) retrospective time frames to evaluate fatigue experience, varying from the preceding seven days to the past four weeks, and potentially lead to recall bias. Third, in research as well as clinical practice, they are usually administered at only one and often arbitrary moment in time, which may fail to capture informative variability in fatigue across time and context. Finally, they may not be validated in HD patients. ${ }^{64}$ Due to these limitations, conventional fatigue measurement instruments may not adequately capture the nature of fatigue, its variability over time and the factors that may be related to that variability (e.g. psychological, social, contextual or behavioural factors). ${ }^{66-68}$ Moreover, their reliability to reflect fatigue experience in 'real-time' and 
'real-world' settings may be questioned, as they are usually applied in artificial settings and inquire fatigue retrospectively, thereby risking ecological and recall bias, respectively. ${ }^{69,70}$ Implementing novel measuring methods that allow capturing fatigue in HD patients in the flow of daily life, its variability and the factors contributing to changes in that variability, is warranted in order to develop effective therapies in the future.

The Experience Sampling Methodology (ESM), also referred to as Ecological Momentary Assessment (EMA), is an innovative measurement method that overcomes the constraints of conventional fatigue scales. The use of ESM is promising to advance the understanding of fatigue in HD patients. ESM is a structured diary technique that allows measuring symptom experience in 'real-time' (i.e. the momentary aspect of EMA) and in individuals' 'real-life' (i.e. the ecological aspect of EMA). ${ }^{68}$ As such, ESM overcomes recall bias and improves ecological validity. ${ }^{68}$ Moreover, ESM consists of repeatedly measuring symptoms over time (e.g. multiple times a day during several consecutive days) and across situations (e.g. in different environments) in the flow of daily life, ${ }^{68}$ while taking into account contextual (e.g. social company, location, daily activities) and psychological factors (e.g. mood, stress or anxiety) that may influence momentary symptom experience. ${ }^{68}$ As such, ESM allows identifying symptom variability and factors that may be related to that variability. ${ }^{68}$ Hence, ESM captures the film rather than a single photograph of symptoms in daily life. ${ }^{71}$ Temporal sequences of symptom experience are documented, which may lead to the identification of antecedents or triggers of events as well as their behavioural consequences. ${ }^{68}$ Moreover, ESM data can be meaningfully interpreted at both the group level (e.g. a cohort of HD patients) and the individual level. ${ }^{72}$ ESM thus allows identifying variables that may explain differences between and within individuals, paving the way towards more personalized interventions. For instance, whereas physical activity may be followed by an increase in fatigue for one person, another person may feel more energetic after physical activity. A better insight in these person-specific relationships is essential in the design of effective person-specific interventions. In other words, ESM allows developing treatment options that work for everyone, rather than for the 'average' patient. Furthermore, digital data collection can help to reduce non-compliance of actual symptom ratings, as a high level of fake compliance has been reported due to backfilling of retrospective paper-and-pencil diaries. ${ }^{73}$ Finally, due to technological advancement, 
there is an increasing number of possibilities to objectively monitor physiological and behavioural parameters in real-time as well, such as heart rate or physical activity by means of daily step count, when embedded in wearable devices. Despite ESM offering many advantages for symptom assessment and its implementation rising in other clinical fields (e.g. in psychopathologies and psychosomatic disorders), the use of ESM in HD patients is still limited. The benefits of applying an ESM to assess fatigue in HD patients will be investigated in the third part of this thesis.

\section{Outline of this thesis}

The first part of this thesis (chapter 2 and 3) discusses the biological basis of fatigue in HD patients, with a specific focus on the hypothesis that fatigue in HD patients may be triggered by inflammation. Chapter 2 focuses on the interrelationship of serum interleukin-6 and endotoxin levels with general fatigue in HD patients. Additionally, the possibility of IL- 6 as a common mediator for fatigue and depressive symptoms in HD patients is explored. In chapter $\mathbf{3}$, we zoom in on postdialysis fatigue specifically and investigate whether HD treatment may trigger an inflammatory response that could explain acute fatigue after treatment. Three pro-inflammatory cytokines, IL-1 $\beta$, IL- 6 and TNF- $\alpha$, and one anti-inflammatory cytokine, IL-10, are highlighted.

The second part of this thesis (chapter 4 and 5) brings into focus psychological and contextual variables that may be related to fatigue experience and its variability in HD patients. Chapter 4 assesses the diurnal course of fatigue in HD patients and its relation with the HD treatment. It is also investigated whether depressive symptoms may be related to this diurnal fatigue variability. Lastly, it is explored whether classical conditioning may explain anticipatory HD-related fatigue, that is, whether feeling tired prior to HD treatment may be - in part - a learned response. In chapter 5, we investigate whether spontaneous daily physical activity, measured by means of a wearable motion sensor recording daily step count, is related to fatigue or depressive mood in HD patients.

The third part of this thesis (chapter 6 and 7) addresses the added value of the Experience Sampling Method (ESM) to assess fatigue in HD patients and compares ESM with conventional retrospective measurement instruments. On the one hand, we 
show how ESM, by means of 'real-time' measurements in HD patients' natural environments, can provide detailed insight in the diurnal variability of fatigue and related psychological, social and behavioural variables (chapter 6). On the other hand, we compare ESM with conventional retrospective questionnaires, and investigate whether retrospective symptom evaluation may lead to different response patterns relative to 'real-time' symptom assessment, supporting the theoretical position that ESM and retrospective instruments may reflect different types of information (chapter 7).

In chapter 8, the general discussion, the main findings of the previous chapters are recapitulated, interpreted and put into perspective. Finally, considerations about the methods, implications for clinical practice and suggestions for future research are formulated. 


\section{References}

1. Webster AC, Nagler EV, Morton RL, Masson P. Chronic Kidney Disease. Lancet (London, England). 2017;389(10075):1238-1252.

2. KDOQI. KDOQI Clinical Practice Guideline and Clinical Practice Recommendations for anemia in chronic kidney disease: 2007 update of hemoglobin target. American journal of kidney diseases : the official journal of the National Kidney Foundation. 2007;50(3):471-530.

3. Hill NR, Fatoba ST, Oke JL, et al. Global Prevalence of Chronic Kidney Disease - A Systematic Review and Meta-Analysis. PloS one. 2016;11(7):e0158765.

4. Mathers CD, Loncar D. Projections of global mortality and burden of disease from 2002 to 2030. PLoS medicine. 2006;3(11):e442.

5. Liyanage $T$, Ninomiya $T$, Jha $V$, et al. Worldwide access to treatment for end-stage kidney disease: a systematic review. Lancet (London, England). 2015;385(9981):1975-1982.

6. Heaf J. Current trends in European renal epidemiology. Clin Kidney J. 2017;10(2):149-153.

7. Kramer A, Pippias M, Noordzij M, et al. The European Renal Association - European Dialysis and Transplant Association (ERA-EDTA) Registry Annual Report 2015: a summary. Clin Kidney J. 2018;11(1):108-122.

8. United States Renal Data System. USRDS annual data report: Epidemiology of kidney disease in the United States. . 2018; https://www.usrds.org/2018/view/Default.aspx.

9. Weisbord SD, Fried LF, Arnold RM, et al. Prevalence, severity, and importance of physical and emotional symptoms in chronic hemodialysis patients. Journal of the American Society of Nephrology : JASN. 2005;16(8):2487-2494.

10. Davison SN, Jhangri GS. Impact of pain and symptom burden on the health-related quality of life of hemodialysis patients. J Pain Symptom Manage. 2010;39(3):477-485.

11. Bossola M, Vulpio C, Tazza L. Fatigue in chronic dialysis patients. Seminars in dialysis. 2011;24(5):550555.

12. Urquhart-Secord R, Craig JC, Hemmelgarn B, et al. Patient and Caregiver Priorities for Outcomes in Hemodialysis: An International Nominal Group Technique Study. American journal of kidney diseases : the official journal of the National Kidney Foundation. 2016;68(3):444-454.

13. Ju A, Unruh M, Davison S, et al. Establishing a Core Outcome Measure for Fatigue in Patients on Hemodialysis: A Standardized Outcomes in Nephrology-Hemodialysis (SONG-HD) Consensus Workshop Report. American journal of kidney diseases : the official journal of the National Kidney Foundation. 2018;72(1):104-112.

14. Riley WT, Rothrock N, Bruce B, et al. Patient-reported outcomes measurement information system (PROMIS) domain names and definitions revisions: further evaluation of content validity in IRTderived item banks. Qual Life Res. 2010;19(9):1311-1321.

15. Hardy SE, Studenski SA. Qualities of fatigue and associated chronic conditions among older adults. $J$ Pain Symptom Manage. 2010;39(6):1033-1042.

16. Bossola M, Di Stasio E, Antocicco M, Tazza L. Qualities of fatigue in patients on chronic hemodialysis. Hemodialysis international. International Symposium on Home Hemodialysis. 2013;17(1):32-40.

17. Jhamb M, Weisbord SD, Steel JL, Unruh M. Fatigue in patients receiving maintenance dialysis: a review of definitions, measures, and contributing factors. American journal of kidney diseases : the official journal of the National Kidney Foundation. 2008;52(2):353-365.

18. Sklar AH, Riesenberg LA, Silber AK, Ahmed W, Ali A. Postdialysis fatigue. American journal of kidney diseases : the official journal of the National Kidney Foundation. 1996;28(5):732-736.

19. Shen J, Barbera J, Shapiro CM. Distinguishing sleepiness and fatigue: focus on definition and measurement. Sleep medicine reviews. 2006;10(1):63-76. 
20. Artom M, Moss-Morris R, Caskey F, Chilcot J. Fatigue in advanced kidney disease. Kidney international. 2014;86(3):497-505.

21. Bonner A, Wellard S, Caltabiano M. Levels of fatigue in people with ESRD living in far North Queensland. J Clin Nurs. 2008;17(1):90-98.

22. Horigan AE. Fatigue in hemodialysis patients: a review of current knowledge. J Pain Symptom Manage. 2012;44(5):715-724.

23. Bossola M, Luciani G, Tazza L. Fatigue and its correlates in chronic hemodialysis patients. Blood purification. 2009;28(3):245-252.

24. O'Sullivan D, McCarthy G. An exploration of the relationship between fatigue and physical functioning in patients with end stage renal disease receiving haemodialysis. J Clin Nurs. 2007;16(11c):276-284.

25. Jhamb M, Pike F, Ramer S, et al. Impact of fatigue on outcomes in the hemodialysis (HEMO) study. American journal of nephrology. 2011;33(6):515-523.

26. McCann K, Boore JR. Fatigue in persons with renal failure who require maintenance haemodialysis. Journal of advanced nursing. 2000;32(5):1132-1142.

27. Williams AG, Crane PB, Kring D. Fatigue in African American women on hemodialysis. Nephrology nursing journal : journal of the American Nephrology Nurses' Association. 2007;34(6):610-617, 644; quiz 618.

28. Liu HE. Fatigue and associated factors in hemodialysis patients in Taiwan. Research in nursing \& health. 2006;29(1):40-50.

29. Morsch CM, Gonçalves LF, Barros E. Health-related quality of life among haemodialysis patients-relationship with clinical indicators, morbidity and mortality. J Clin Nurs. 2006;15(4):498-504.

30. Bossola M, Luciani G, Giungi S, Tazza L. Anorexia, fatigue, and plasma interleukin-6 levels in chronic hemodialysis patients. Renal failure. 2010;32(9):1049-1054.

31. Lobbedez T, Desbordes E, Joly F, Ficheux M, Henri P, Ryckelynck JP. [Fatigue in elderly patients on dialysis]. Nephrologie \& therapeutique. 2008;4(7):584-589.

32. Brunier GM, Graydon J. The influence of physical activity on fatigue in patients with ESRD on hemodialysis. ANNA journal. 1993;20(4):457-461; discussion 462, 521.

33. Garcia RSA, Lucinda LMF, Ramos FA, et al. Factors Associated With Functional Capacity in Hemodialysis Patients. Artificial organs. 2017;41(12):1121-1126.

34. Sheshadri A, Kittiskulnam P, Johansen KL. Higher Physical Activity Is Associated With Less Fatigue and Insomnia Among Patients on Hemodialysis. Kidney international reports. 2019;4(2):285-292.

35. Johansen $\mathrm{KL}$, Chertow GM, Ng AV, et al. Physical activity levels in patients on hemodialysis and healthy sedentary controls. Kidney international. 2000;57(6):2564-2570.

36. Leinau L, Murphy TE, Bradley E, Fried T. Relationship between conditions addressed by hemodialysis guidelines and non-ESRD-specific conditions affecting quality of life. Clinical journal of the American Society of Nephrology : CJASN. 2009;4(3):572-578.

37. Dantzer R. Cytokine-induced sickness behavior: mechanisms and implications. Annals of the New York Academy of Sciences. 2001;933:222-234.

38. Moreland LW, Genovese MC, Sato R, Singh A. Effect of etanercept on fatigue in patients with recent or established rheumatoid arthritis. Arthritis and rheumatism. 2006;55(2):287-293.

39. Davis MC, Zautra AJ, Younger J, Motivala SJ, Attrep J, Irwin MR. Chronic stress and regulation of cellular markers of inflammation in rheumatoid arthritis: implications for fatigue. Brain Behav Immun. 2008;22(1):24-32.

40. Yasumoto S, Imayama S, Hori Y. Increased serum level of interleukin-6 in patients with psoriatic arthritis and thrombocytosis. The Journal of dermatology. 1995;22(10):718-722.

41. Flachenecker P, Bihler I, Weber F, Gottschalk M, Toyka KV, Rieckmann P. Cytokine mRNA expression in patients with multiple sclerosis and fatigue. Multiple sclerosis (Houndmills, Basingstoke, England). 2004;10(2):165-169. 
42. Heesen C, Nawrath L, Reich C, Bauer N, Schulz KH, Gold SM. Fatigue in multiple sclerosis: an example of cytokine mediated sickness behaviour? Journal of neurology, neurosurgery, and psychiatry. 2006;77(1):34-39.

43. Saligan LN, Kim HS. A systematic review of the association between immunogenomic markers and cancer-related fatigue. Brain Behav Immun. 2012;26(6):830-848.

44. Schubert C, Hong S, Natarajan L, Mills PJ, Dimsdale JE. The association between fatigue and inflammatory marker levels in cancer patients: a quantitative review. Brain Behav Immun. 2007;21(4):413-427.

45. Monk JP, Phillips G, Waite R, et al. Assessment of tumor necrosis factor alpha blockade as an intervention to improve tolerability of dose-intensive chemotherapy in cancer patients. J Clin Oncol. 2006;24(12):1852-1859.

46. Leng S, Chaves P, Koenig K, Walston J. Serum interleukin-6 and hemoglobin as physiological correlates in the geriatric syndrome of frailty: a pilot study. Journal of the American Geriatrics Society. 2002;50(7):1268-1271.

47. Dantzer R, O'Connor JC, Freund GG, Johnson RW, Kelley KW. From inflammation to sickness and depression: when the immune system subjugates the brain. Nature reviews. Neuroscience. 2008;9(1):46-56.

48. Morris G, Berk M, Walder K, Maes M. Central pathways causing fatigue in neuro-inflammatory and autoimmune illnesses. BMC Med. 2015;13:28.

49. Dantzer R, Heijnen CJ, Kavelaars A, Laye S, Capuron L. The neuroimmune basis of fatigue. Trends Neurosci. 2014;37(1):39-46.

50. Dantzer R, Kelley KW. Twenty years of research on cytokine-induced sickness behavior. Brain Behav Immun. 2007;21(2):153-160.

51. Karshikoff B, Sundelin T, Lasselin J. Role of Inflammation in Human Fatigue: Relevance of Multidimensional Assessments and Potential Neuronal Mechanisms. Frontiers in immunology. 2017;8:21.

52. Norheim KB, Jonsson G, Omdal R. Biological mechanisms of chronic fatigue. Rheumatology (Oxford, England). 2011;50(6):1009-1018.

53. Piepoli M, Clark AL, Volterrani M, Adamopoulos S, Sleight P, Coats AJ. Contribution of muscle afferents to the hemodynamic, autonomic, and ventilatory responses to exercise in patients with chronic heart failure: effects of physical training. Circulation. 1996;93(5):940-952.

54. Yao Q, Lindholm B, Stenvinkel P. Inflammation as a cause of malnutrition, atherosclerotic cardiovascular disease, and poor outcome in hemodialysis patients. Hemodialysis international. International Symposium on Home Hemodialysis. 2004;8(2):118-129.

55. Kaysen GA. The microinflammatory state in uremia: causes and potential consequences. Journal of the American Society of Nephrology : JASN. 2001;12(7):1549-1557.

56. Bambauer R, Schmidt R, Falkenhagen D, Walther J, Jung WK. Sterile and endotoxin free dialysis fluid for hemodialysis. Biomaterials, artificial cells, and immobilization biotechnology : official journal of the International Society for Artificial Cells and Immobilization Biotechnology. 1991;19(1):71-83.

57. Wong J, Vilar E, Farrington K. Endotoxemia in end-stage kidney disease. Seminars in dialysis. 2015;28(1):59-67.

58. Vanholder R, Ringoir S, Dhondt A, Hakim R. Phagocytosis in uremic and hemodialysis patients: a prospective and cross sectional study. Kidney international. 1991;39(2):320-327.

59. Pae CU, Lim HK, Han C, et al. Fatigue as a core symptom in major depressive disorder: overview and the role of bupropion. Expert review of neurotherapeutics. 2007;7(10):1251-1263.

60. Howren MB, Lamkin DM, Suls J. Associations of depression with C-reactive protein, IL-1, and IL-6: a meta-analysis. Psychosomatic medicine. 2009;71(2):171-186. 
61. Palmer S, Vecchio M, Craig JC, et al. Prevalence of depression in chronic kidney disease: systematic review and meta-analysis of observational studies. Kidney international. 2013;84(1):179-191.

62. Surawy C, Hackmann A, Hawton K, Sharpe M. Chronic fatigue syndrome: a cognitive approach. Behaviour research and therapy. 1995;33(5):535-544.

63. van Kessel K, Moss-Morris R. Understanding multiple sclerosis fatigue: a synthesis of biological and psychological factors. J Psychosom Res. 2006;61(5):583-585.

64. Ju A, Unruh ML, Davison SN, et al. Patient-Reported Outcome Measures for Fatigue in Patients on Hemodialysis: A Systematic Review. American journal of kidney diseases : the official journal of the National Kidney Foundation. 2018;71(3):327-343.

65. Whitehead L. The measurement of fatigue in chronic illness: a systematic review of unidimensional and multidimensional fatigue measures. J Pain Symptom Manage. 2009;37(1):107-128.

66. Abdel-Kader K, Jhamb M, Mandich LA, et al. Ecological momentary assessment of fatigue, sleepiness, and exhaustion in ESKD. BMC nephrology. 2014;15:29.

67. Picariello F, Moss-Morris R, Macdougall IC, Chilcot J. 'It's when you're not doing too much you feel tired': A qualitative exploration of fatigue in end-stage kidney disease. British journal of health psychology. 2018;23(2):311-333.

68. Shiffman S, Stone AA, Hufford MR. Ecological momentary assessment. Annual review of clinical psychology. 2008;4:1-32.

69. Van den Bergh O, Walentynowicz M. Accuracy and bias in retrospective symptom reporting. Current opinion in psychiatry. 2016;29(5):302-308.

70. Ben-Zeev D, McHugo GJ, Xie H, Dobbins K, Young MA. Comparing retrospective reports to realtime/real-place mobile assessments in individuals with schizophrenia and a nonclinical comparison group. Schizophrenia bulletin. 2012;38(3):396-404.

71. Myin-Germeys I, Oorschot M, Collip D, Lataster J, Delespaul P, van Os J. Experience sampling research in psychopathology: opening the black box of daily life. Psychological medicine. 2009;39(9): 1533-1547.

72. van Os J, Verhagen S, Marsman A, et al. The experience sampling method as an mealth tool to support self-monitoring, self-insight, and personalized health care in clinical practice. Depression and anxiety. 2017;34(6):481-493.

73. Stone AA, Shiffman S, Schwartz JE, Broderick JE, Hufford MR. Patient compliance with paper and electronic diaries. Controlled clinical trials. 2003;24(2):182-199. 

Part I

The role of inflammation in fatigue symptoms in chronic haemodialysis patients 



\section{Chapter 2}

Serum interleukin-6 and endotoxin levels and their relationship with fatigue and depressive symptoms in patients on chronic haemodialysis

Brys ADH*, Di Stasio E*, Lenaert B, Sanguinetti M, Picca A, Calvani R, Marzetti E, Gambaro G, Bossola M. "shared first authorship

Published in Cytokine. 2020;125:154823 


\begin{abstract}

\section{Background}

Little is known about the underlying aetiology of fatigue in haemodialysis (HD) patients apart from a significant association and overlapping symptomatology with depressive symptoms. Growing evidence exists for the involvement of inflammation, by pro-inflammatory cytokines, in the development of fatigue in other inflammatory diseases. In HD patients, increased exposure to bacterial endotoxins may contribute to an inflammatory response and potentially lead to fatigue. We therefore aimed (i) to assess the interrelationship between serum endotoxin (EA) levels, interleukin-6 (IL-6) levels and fatigue in HD patients; (ii) to evaluate whether there is a relationship between depression and inflammation as well and (iii) to what extent depression and fatigue are related to each other.
\end{abstract}

\title{
Methods
}

Fatigue and depressive symptoms in daily life were assessed in 59 individuals using the SF-36 vitality subscale and the Geriatric Depression Scale. Blood samples were collected on a mid-week dialysis session to determine EA levels, through the Endotoxin Activity Assay (EAA ${ }^{\mathrm{TM}}$ ), and IL-6 concentrations, through the commercially available Abcam ELISA (Enzyme-Linked Immunosorbent Assay) kit.

\section{Results}

EA, IL-6 levels and depressive symptoms were significantly correlated with fatigue. EA levels and depressive symptoms are predictor variables of fatigue, explaining $31 \%$ of its variance. However, EA and IL-6 were not significantly associated with depression.

\section{Conclusions}

Fatigue in HD patients may be related to endotoxemia and inflammation through IL-6. Furthermore, fatigue is significantly associated with depressive symptoms. Future research into the causative interrelationship of inflammation, fatigue and depression in HD patients might lead to potential targets for therapeutic strategies. 


\section{Introduction}

The prevalence of haemodialysis (HD) treatment in Europe and the U.S. is estimated to be 465 and 1437 per million population, respectively. ${ }^{1,2}$ Fatigue is one of the most frequent and burdensome symptoms among haemodialysis patients, affecting their health related quality of life substantially. ${ }^{3}$

However, fatigue in patients on chronic HD treatment remains a poorly understood symptom. This is in part due to the inherently subjective nature of fatigue, making it difficult to capture and define. Dialysis patients may say they feel tired, weak, exhausted, worn-out, heavy or slow whereas health professionals may use other terms such as asthenia, lassitude, exercise intolerance, lack of energy and weakness to describe fatigue. ${ }^{4}$ Moreover, different fatigue qualities can be distinguished, such as physical (weakness, tiredness, lack of energy) and mental (emotional, cognitive) fatigue, with the former being reported more frequently in HD populations. ${ }^{5}$

Furthermore, the aetiology of fatigue in HD patients is complex and likely multidetermined. Physiological (e.g. uraemia, anaemia, inflammation, comorbidities), psychological and behavioural (e.g. stress, depression, anxiety, sleep), sociodemographic (e.g. age, sex, social support), and treatment variables (e.g. dialysis adequacy, mode or frequency) may all contribute to fatigue, although current evidence is largely inconsistent. ${ }^{6,7}$ Only a significant association with depressive symptoms has been consistently reported, but no causal relationship has yet been demonstrated. ${ }^{8,9}$ This association may be due to overlapping symptoms, as a result of which screening instruments for fatigue and depression may partly measure the same phenomenon. Further, fatigue and depression may influence each other in a reciprocal relationship or they both could be different expressions of a common physiopathological pathway. However, the precise underlying physiopathology is still being investigated.

Interestingly, there is growing evidence for the involvement of inflammation in the development of pathological fatigue in infectious diseases, cancer, neurodegenerative diseases (e.g. multiple sclerosis) and auto-immune diseases (e.g. rheumatoid arthritis). ${ }^{10-14}$ In this study we will investigate the relationship between fatigue in HD 
patients and inflammation by means of serum interleukin-6 (IL-6) levels and serum endotoxin (EA) levels.

IL-6, a pro-inflammatory cytokine, likewise IL-1 and tumour necrosis factor $\alpha$ (TNF- $\alpha$ ) may interfere with the central nervous system causing non-specific sickness symptoms, often referred to as 'sickness behaviour'. ${ }^{15}$ These may include fatigue and decreased physical activity, reduced ability to concentrate and an altered mood state, withdrawal from social interaction and loss of appetite. ${ }^{16,17}$ It may be seen as a biologically triggered coping mechanism by inducing rest in order to conserve energy and thus allow recovery to take place. ${ }^{18}$ Similarly, depression might derive from immunological roots as its development has also been associated with cytokine-tobrain communication. ${ }^{19,20}$ As such, depression has been proposed to constitute a continuation of the altered mood state already present in sickness behaviour. ${ }^{21,22}$

Approximately $30-50 \%$ of patients with end stage kidney disease (ESKD), undergoing HD treatment, show evidence of an inflammatory response. ${ }^{23}$ Multiple factors may contribute to this chronic inflammatory state. For instance, renal failure itself leads to the accumulation of pro-inflammatory components, reduced plasma antioxidant activity and increased oxidative stress. Additionally, the interaction of individuals' blood with bioincompatible dialysis membranes during the HD treatment may cause activation of circulating mononuclear cells, stimulating cytokine production. Furthermore, the quality of the dialysis fluid, such as microbial or endotoxin contaminated dialysis water, may trigger transmembrane stimulation of circulating immune cells. ${ }^{24,25}$ Endotoxins are soluble complexes of lipopolysaccharide (LPS) located on the outer membrane of Gram-negative bacteria capable of inducing a systemic inflammatory reaction through the production of cytokines, such as IL-6, IL-1 and TNF- $\alpha .^{26,27}$ As a consequence, ultrapure and endotoxin-free (i.e. $<0.03$ IU endotoxin unit/ml) dialysis water is recommended by the European Renal Association. $^{28}$

Moreover, the intestinal barrier in HD patients is disrupted by the uremic environment, allowing translocation of intestinal microbiota, mainly consisting of Gram-negative bacteria. ${ }^{26}$ Finally, HD patients themselves are more susceptible to infections due to impaired humoral and cellular immunity, vascular accesses and often co-occurring diabetes mellitus. ${ }^{29}$ Together, these factors potentially promote the 
inflammatory status commonly observed in the haemodialysis population. Indeed, several reports demonstrated elevated levels of IL-1, TNF- $\alpha$, IL- 6 and serum endotoxins (EA) in HD patients. ${ }^{30-34}$

Only a limited number of studies investigated the relation between fatigue in HD patients and pro-inflammatory cytokines. These studies showed a significant association between fatigue and IL- 6 levels as well as a significant association between IL- 6 and serum EA levels, suggesting endotoxemia may play a role in the development of chronic inflammation and fatigue in HD patients. ${ }^{35-37}$ However, the relationship between serum EA and fatigue in HD patients, mediated by IL-6, has not been thoroughly investigated in previous research. We therefore aimed to (i) assess the interrelationship between serum EA, IL-6 and fatigue in haemodialysis patients, (ii) to assess the relationship between inflammation and depression as well and (iii) investigate the association between depression and fatigue in HD patients.

\section{Subjects and methods}

\section{Study population}

Data in this study were collected as part of a research project involving multiple research questions. Results demonstrating that serum EA levels were intermediately or highly present in half of the studied HD patients and strongly associated with IL-6 levels have recently been published. ${ }^{38}$

All prevalent patients of the Haemodialysis Unit of the Catholic University of Rome (Italy) on a chronic haemodialysis treatment for at least six months during the spring and summer of 2016 were approached for this observational study. Exclusion criteria were: $<18$ years of age, acute or chronic infection, sepsis, antibiotic therapy in the last two weeks, diagnosed auto-immune disorder, neurodegenerative disease or active cancer. The local ethics committee approved the study protocol (P/606/CE/2011). All participants gave their informed consent before enrollment in the study. The study was carried out in accordance with the Declaration of Helsinki. 


\section{Haemodialysis treatment}

Patients received bicarbonate haemodialysis treatment for four hours, thrice weekly. The blood flow ranged from 250 to $300 \mathrm{ml} / \mathrm{min}$ with a dialysis rate flow of $500 \mathrm{ml} / \mathrm{min}$. High permeability membranes were used. To prepare the dialysate a Culligan $\mathrm{RO}^{2}$ Bi-Osmosis system (Culligan, Buckinghamshire, United Kingdom) is used in our centre, resulting in ultrapure dialysis fluid with $<0.03$ endotoxin unit/ml, assessed through the Limulus amebocyte lysate ( $L A L)$ test. The LAL test is a highly sensible test to quantify endotoxins and, therefore, the most widely used method for endotoxin detection in non biological samples. The principle of the LAL assay is based on a gelation reaction resulting in the formation of a clot, following activation of a cascade of enzymes by endotoxin. ${ }^{26}$ In our institution, the LAL assay is performed monthly.

\section{Data collection}

\section{Assessment of fatigue and depressive symptoms}

The vitality subscale of the Italian version of the SF-36 health survey, a 36-item selfreport questionnaire assessing physical and psychological health concepts in eight subscales (i.e. physical functioning, role limitations due to physical problems, bodily pain, general health perceptions, vitality, social functioning, role limitations due to emotional problems and general mental health), was administered to evaluate fatigue. The SF-36 vitality subscale (SF-36 VS) is a four-item questionnaire with a sixpoint frequency scale and assesses to what extent individuals felt full of energy versus tired and worn out during the last month, as fatigue is considered to be the opposite pole of a continuum of vitality and fatigue. Individual item-score can range from 1 (all of the time) to 6 (none of the time). The total score of the vitality subscale is transformed into a score ranging from 0 to 100 with a higher score indicating greater vitality. ${ }^{39}$ The SF-36 shows good discriminant validity (i.e. significant differences in scores (all subscales) of patients with serious and minor medical conditions and psychiatric conditions) and the SF-36 vitality subscale shows acceptable internal consistency. ${ }^{40}$ The SF-36 VS is one of the most frequently used measuring instruments to evaluate fatigue in haemodialysis patients. ${ }^{7,41}$ 
The Italian version of the Geriatric Depression Scale (GDS) was administered as a screening tool for depressive symptoms during the past week. The GDS has been shown to have good psychometric properties with an internal consistency of 0.84 (Cronbach's alpha), concurrent validity of 0.83 and a test-re-test reliability of $0.91 .^{42}$ The GDS is a self-report questionnaire composed of 30 items that are scored dichotomously and excludes somatic and psychotic symptoms. Therefore, the GDS allows evaluating depressive symptoms in HD patients in isolation from symptoms related to the pathophysiology or treatment of ESKD such as loss of appetite or sleep disorders. Total scores range from 0-30. Scores of 11-20 and 21-30 indicate mild and severe depressive mood, respectively. ${ }^{42}$

\section{Laboratory measurements}

A blood sample was collected from all patients under sterile conditions from a peripheral vein during the mid-week dialysis session. Laboratory parameters (haemoglobin, albumin, creatinine, urea, calcium, phosphorus, parathyroid hormone, C-reactive protein (CRP)) were measured within four hours of specimen collection using Advia2120i instruments (Advia Centaur XPT, Advia Centaur XPT Siemens Healthineers) according to the manufacturer's instructions at the Laboratory of Clinical Chemistry - Fondazione Policlinico A. Gemelli IRCSS, UCSC Rome. Serum Interleukin-6 was determined by a commercially available human high sensitivity in vitro ELISA (Enzyme-Linked Immunosorbent Assay) kit (Abcam, Cambridge, UK; sensitivity: $<0.8 \mathrm{pg} / \mathrm{ml}$; Intra-assay coefficient of variation: $4.4 \%$ ) and a AHSI 880TS microplate absorbance reader (AHSI S.p.A., Bernareggio, MB, Italy) according to the manufacturer's instructions. Serum endotoxin levels were determined by an Endotoxin Activity Assay (EAA) and a chemiluminometer (EAA ${ }^{\mathrm{TM}}$ and Smart Line TL Luminometer, Spectral Diagnostics Inc., Toronto, ON, Canada). The EAA measures the oxidative response of neutrophils to LPS-antibody complexes. The EAA uses a highly specific IgM antibody for the most preserved structural component of endotoxin, Lipid-A, allowing to specifically measure endotoxin activity in human whole blood (which is not possible using the LAL assay). The EAA is the only licensed assay to diagnose endotoxemia by the United States Food and Drug Administration (USFDA). ${ }^{26,43}$ 


\section{Baseline and clinical data}

The following demographic, clinical and laboratory information was obtained from medical records at the moment of inclusion in the study: age, gender, dialysis vintage, type and number of comorbidities assessed by the Charlson Comorbidity Index, ${ }^{44}$ self reported functional status via de Activity of daily living scale, ${ }^{45}$ dialysis dose as evaluated by $K t / \mathrm{V}$.

\section{Procedure}

After giving their informed consent, each participant received the Italian version of SF36 questionnaire and GDS along with verbal instructions from the attending physician. The questionnaires were completed at home and turned in two days later during the next dialysis session. Blood samples were provided within the same week, during the mid-week dialysis session.

\section{Statistical analyses}

Statistical analysis was performed by using the Statistical Package for Social Science (SPSS). Continuous variables were expressed as mean, standard deviation (SD) and range, categorical variables displayed as frequencies and statistical significance was assessed at $p<0.05$. In order to investigate correlations between variables Spearman's rho correlation coefficients $\left(r_{s}\right)$ were calculated. Moreover, we performed a multiple linear regression analysis (backward method) to estimate the predictability of fatigue by serum EA levels, IL-6 concentration and depressive symptoms while accounting for potentially related variables that were chosen based on prior literature and expert opinion. These included age, gender, dialysis vintage and haemoglobin. A backward stepwise regression analysis starts with all chosen predictor variables included in the model. The final, reduced model contains only the variables that independently predict variance in the dependent measure (i.e. fatigue severity). The predictability of the models was measured by means of adjusted $R^{2}$ values. 


\section{Results}

\section{Sample characteristics}

Sixty-two patients were approached of whom sixty-one provided informed consent (98\% consent rate). Subsequently, two patients were excluded from the analysis because they turned in the GDS questionnaire incomplete. Baseline demographical, clinical and laboratory characteristics of the remaining 59 patients are reported in Table 2.1. The SF-36 VS mean score (SD) was 58.6 (20.7) and the GDS mean score (SD) 9.7 (6.3). Most subjects were under regular treatment with recombinant human erythropoietin, antihypertensive medications ( $\beta$-blockers, calcium channel blockers, angiotensin-converting enzyme inhibitors) and other commonly used drugs such as 1,25-dihydroxyvitamin $D$, phosphate and potassium binders. None of them received corticosteroids, cytotoxic or antidepressant drugs at the time of the study.

Table 2.1 Baseline demographical, clinical and laboratory characteristics ( $N=59)$.

\begin{tabular}{lc}
\hline Characteristics & Mean \pm SD [range] / frequency \\
\hline Age (years) & $61 \pm 15[25-85]$ \\
Sex (male) & $64 \%$ \\
BMI (kg/m²) & $25.8 \pm 8.3$ \\
Dialysis vintage (months) & $66 \pm 60$ \\
Charlson Comorbidity lndex & $2.8 \pm 2.4[0-9]$ \\
Activity of Daily Living & $5.6 \pm 0.9[3-6]$ \\
SF-36 vitality subscale & $58.6 \pm 20.7[15-90]$ \\
Geriatric Depression Scale & $9.7 \pm 6.3[0-24]$ \\
Interdialytic weight gain (kg) & $3.0 \pm 1.1$ \\
Kt/V & $1.4 \pm 0.3$ \\
Haemoglobin (g/dl) & $11.0 \pm 0.9$ \\
Serum albumin (mg/dl) & $3.7 \pm 0.1$ \\
Serum creatinine (mg/dl) & $10.0 \pm 1.9$ \\
Serum urea (mg/dl) & $83 \pm 18$ \\
PTH (mg/dl) & $298 \pm 207$ \\
Calcium (mg/dl) & $9.2 \pm 0.6$ \\
Phosphorus (mg/dl) & $5.1 \pm 1.7$ \\
CRP (mg/ml) & $30.5 \pm 26.6[0.0-92.0]$ \\
Serum Interleukin-6 (pg/ml) & $13.0 \pm 13.7[0.2-68.0]$ \\
Serum Endotoxin (units) & $0.4 \pm 0.3[0.1-1.1]$ \\
\hline
\end{tabular}

$K t / V$ : urea clearance, PTH: Parathyroid hormone, CRP: C-reactive protein 
Serum EA, IL-6 levels and their relation with fatigue and depressive symptoms

Figure 2.1 shows fatigue as function of serum endotoxin concentrations and IL-6 levels (panel $a$ and $b$, respectively). Correlations between the variables under analysis (i.e. serum EA, IL-6, SF-36 VS and GDS) are reported in Table 2.2. IL-6 and serum EA levels are significantly correlated, $r_{s}=0.95, p<0.001$. Interestingly, both of them are significantly inversely associated with the SF-36 VS, $r_{s}=-0.33, p=0.011$ for both, in that higher levels of endotoxemia and inflammation predict a lower SF-36 vitality score, indicating higher levels of fatigue. Furthermore, a significant inverse correlation is found between SF-36 VS and GDS, $r_{s}=-0.50, p<0.001$, demonstrating the interrelationship between fatigue and depressive symptoms. However, neither IL-6 levels nor serum endotoxin concentration is significantly associated with depressive symptoms, $r_{s}=0.23, p=0.087$; and $r_{s}=0.21, p=0.103$, respectively.

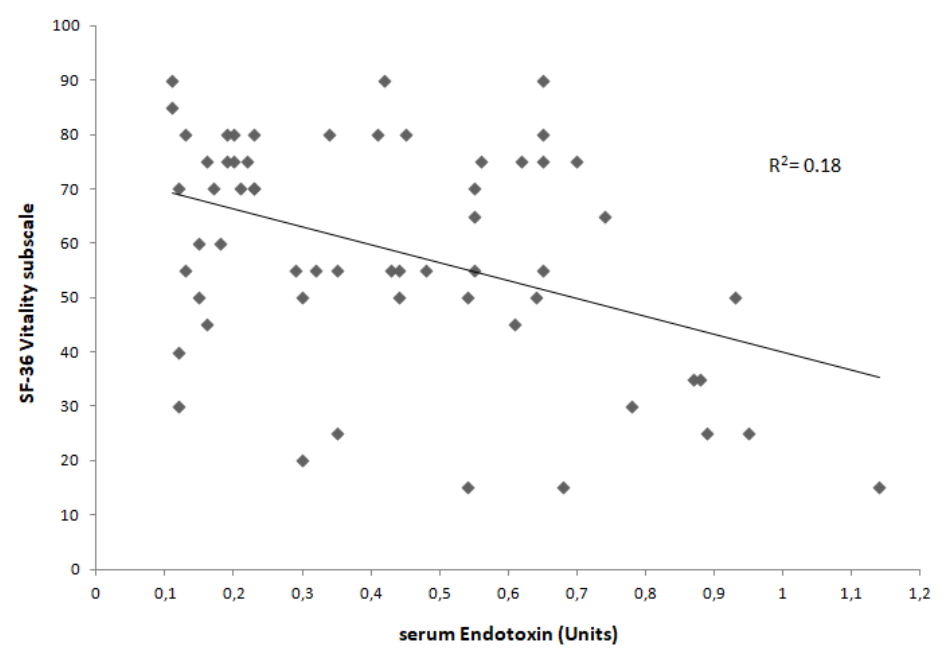

Figure 2.1a Fatigue as function of serum Endotoxin levels ( $N=59)$. 


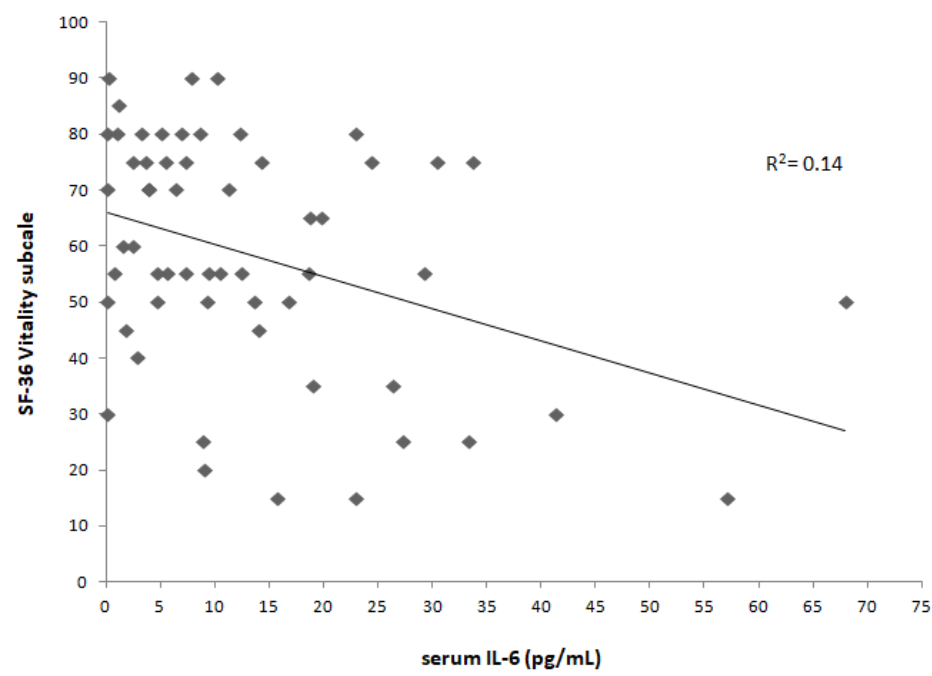

Figure 2.1b Fatigue as function of serum IL-6 levels ( $N=59)$.

Table 2.2 Correlation matrix of principal variables under analysis $(\mathrm{N}=59)$.

\begin{tabular}{|c|c|c|c|c|}
\hline \multicolumn{5}{|c|}{ Spearman's rho Correlation Coefficient [95\% confidence intervals] ( $p)$} \\
\hline & $\begin{array}{c}\text { Serum Endotoxin } \\
\text { (units) }\end{array}$ & $\begin{array}{c}\begin{array}{c}\text { Serum IL-6 } \\
(\mathrm{pg} / \mathrm{ml})\end{array} \\
\end{array}$ & GDS & SF-36 VS \\
\hline $\begin{array}{l}\text { Serum Endotoxin } \\
\text { (units) }\end{array}$ & - & $\begin{array}{c}0.95[0.93,0.96] \\
(<0.001)\end{array}$ & $\begin{array}{c}0.21[-0.04,0.44] \\
(0.103)\end{array}$ & $\begin{array}{c}-0.33[-0.55,-0.09] \\
(0.011)\end{array}$ \\
\hline $\begin{array}{l}\text { Serum IL-6 } \\
(\mathrm{pg} / \mathrm{ml})\end{array}$ & $\begin{array}{c}0.95[0.93,0.96] \\
(<0.001)\end{array}$ & - & $\begin{array}{c}0.23[-0.02,0.45] \\
(0.087)\end{array}$ & $\begin{array}{c}-0.33[-0.56,-0.08] \\
(0.011)\end{array}$ \\
\hline GDS & $\begin{array}{c}0.21[-0.04,0.44] \\
(0.103)\end{array}$ & $\begin{array}{c}0.23[-0.02,0.45] \\
(0.087)\end{array}$ & - & $\begin{array}{c}-0.50[-0.65,-0.33] \\
(<0.001)\end{array}$ \\
\hline SF-36 VS & $\begin{array}{c}-0.33[-0.55,-0.09] \\
(0.011)\end{array}$ & $\begin{array}{c}-0.33[-0.56,-0.08] \\
(0.011)\end{array}$ & $\begin{array}{c}-0.50[-0.65,-0.33] \\
(<0.001)\end{array}$ & - \\
\hline
\end{tabular}

IL-6: Interleukin-6, GDS: Geriatric Depression Scale, SF-36 VS: SF-36 vitality subscale

To test whether serum EA levels, IL-6 levels and depressive symptoms are predictor variables for fatigue, a multiple regression analysis was carried out with SF-36 VS as the dependent variable and serum EA, IL-6 levels and GDS as independent variables, while accounting for potentially related variables (i.e. age, gender, dialysis vintage and haemoglobin). The reduced model shows that only serum endotoxin levels and GDS are independent predictor variables of fatigue in this HD population (Table 2.3). The strong relationship between EA and IL- 6 suggests that cytokines are likely involved in 
modulating the effect of EA on fatigue symptoms. Furthermore, it confirms the interdependence between depression and fatigue. The adjusted $R^{2}$ of 0.31 of the reduced model indicates that endotoxin levels and GDS together explain approximately one third of the variance in fatigue.

Table 2.3 Results of multiple regression analyses with fatigue (SF-36 VS) as dependent variable (N=59).

\begin{tabular}{|c|c|c|c|c|c|}
\hline \multicolumn{6}{|c|}{ Entire regression model: adjusted $\mathrm{R}^{2}=0.28$} \\
\hline Predictor variables & $\boldsymbol{b}$ & SE B & $\boldsymbol{\beta}$ & $t$ & $p$ \\
\hline constant & 93.83 & 31.08 & - & 3.02 & 0.004 \\
\hline Serum Endotoxin (units) & -19.829 & 18.92 & -0.25 & -1.05 & 0.299 \\
\hline Serum IL-6 (pg/ml) & -0.10 & 0.36 & -0.07 & -0.28 & 0.784 \\
\hline GDS & -1.34 & 0.40 & -0.41 & -3.31 & 0.002 \\
\hline Age (years) & -0.02 & 0.17 & -0.01 & -0.09 & 0.931 \\
\hline Gender (female/male) & -3.15 & 4.79 & -0.07 & -0.67 & 0.514 \\
\hline Dialysis vintage (months) & -0.06 & 0.04 & -0.17 & -1.42 & 0.162 \\
\hline Haemoglobin $(\mathrm{mg} / \mathrm{dL})$ & -0.60 & 2.62 & -0.03 & -0.23 & 0.821 \\
\hline \multicolumn{6}{|c|}{ Reduced regression model (backward-stepwise method): adjusted $R^{2}=0.31$} \\
\hline Predictor variables & $\boldsymbol{b}$ & SE B & $\beta$ & $t$ & $p$ \\
\hline constant & 82.59 & 5.11 & - & 16.17 & $<0.001$ \\
\hline Serum Endotoxin (units) & -25.73 & 8.86 & -0.33 & -2.91 & 0.005 \\
\hline GDS & -1.33 & 0.37 & -0.41 & -3.61 & 0.001 \\
\hline
\end{tabular}

$b=$ unstandardized regression coefficient, SE $B=$ standard error of $b, \beta=$ standardized regression coefficient, GDS: Geriatric Depression Scale, IL-6: Interleukin-6, SF-36 VS: SF-36 vitality subscale

\section{Discussion}

Previous results of this research project demonstrated that half of the studied HD patients had intermediate or high serum endotoxin (EA) levels, even though patients with an active, chronic or recently overcome infection had been excluded. ${ }^{38}$ Elevated EA levels in HD patients may be related to endotoxin contaminated dialysis water, a disrupted intestinal barrier with translocation of intestinal microbiota or the use of vascular accesses. ${ }^{24,26}$ Since endotoxins are a potential source of inflammation by cytokines such as IL-6, which in turn have been related to pathological fatigue in other (inflammatory) diseases, we assessed in the present study whether serum endotoxin and IL-6 levels were related to fatigue and depressive symptoms in haemodialysis patients. Furthermore, we evaluated the relationship between fatigue and depressive symptoms. We found that serum EA and IL-6 were significantly correlated with fatigue but not with depressive symptoms. Multiple linear regression revealed that serum endotoxin levels and depressive symptoms independently predicted fatigue, together explaining $31 \%$ of variance in fatigue in this study sample (Table 2.3 ). 
Previous studies in HD patients showed that serum endotoxin levels are associated with IL-6 levels and that IL-6 in turn is associated with fatigue. ${ }^{35-38}$ The current study adds to the literature by demonstrating an additional and significant association between serum EA and fatigue in HD patients. These results suggest that higher serum endotoxin levels predict more fatigue symptoms. Further, figure 1a shows especially low vitality scores for the highest serum endotoxin levels. This may potentially point to a critical endotoxemic threshold that surpasses the body's capacity to cope with inflammation. Taken together, these results may suggest that endotoxemia stimulates an inflammatory response that ultimately leads to the development of fatigue in HD patients. Future research with longitudinal studies is needed to test this hypothesis, and may reveal serum EA or IL-6 to be a prospective factor for fatigue in HD patients. Insofar a causal relationship between serum EA or IL- 6 and fatigue can be demonstrated, these inflammatory markers may become potential targets for therapeutic strategies.

However, only $31 \%$ of the variance of fatigue in these HD patients could be explained by serum EA levels and depressive symptoms. Therefore, other factors may be involved in the onset and maintenance of fatigue in the HD population. This is in line with a recently proposed biopsychosocial model of fatigue in HD patients, in which fatigue may initially be triggered by chemical imbalances, but may eventually be perpetuated by other factors such as psychological (e.g. depression) and social constituents (e.g. poor social support). ${ }^{8}$

In fact, depressive symptoms and fatigue were significantly associated in our study sample. These findings are in line with several previous studies reporting an association between depression and fatigue in HD patients. ${ }^{8}$ With respect to the underlying mechanism, a significant association between IL-6 and depression has been repeatedly reported in patients without kidney disease. ${ }^{20}$ However, in the HD population, results are still mixed. Our study, likewise others, did not find a significant association between IL- 6 and depressive symptoms. ${ }^{46-48}$ Neither did we observe any relationship between serum EA and depressive mood. This latter finding contrasts with previous findings showing a significant association between IL- 6 and depression in HD patients. ${ }^{35,49-51}$ Part of the reason why we did not find this association may be due to the use of the GDS, developed to assess depressive symptoms in geriatric populations. Although our study sample had a median age of 65 years, not all 
participants can be considered to be in the geriatric age range. Further, in contrast to other frequently used measurement instruments for depressive symptoms, such as the Beck Depression Inventory or the Patient Health Questionnaire-9, the GDS does not inquire for loss of appetite or sleep disorders. These complaints are often seen in patients with ESKD undergoing HD treatment without necessarily pointing to the presence of depression. Therefore, it is possible that the GDS allows evaluating depressive symptoms in HD patients in isolation from symptoms related to the pathophysiology or treatment of ESKD.

Whether the inconsistency about the relationship between IL- 6 and depression in HD patients can be explained by the relative small sample sizes of the various studies and the application of different measurement instruments to assess depressive symptoms, needs to be clarified in future studies with larger sample sizes and the implementation of diagnostic interviews to determine the presence of depression.

The strength of the present study is that serum endotoxin levels were assessed with a validated $E A A^{T M}$, specifically measuring endotoxin activity in human whole blood, which the LAL assay is not able to do. Limitations include the rather small study population of a single center. Therefore the generalizability of this study needs to be confirmed in future research. Further, the retrospective assessment of fatigue and depressive symptoms may be liable to a recall bias. Finally, our findings are based on correlations approaches and, therefore, we cannot draw any conclusions about causality. Investigating the relationship of other pro-and anti-inflammatory cytokines, such as IL-1, TNF- $\alpha$, IL-10 with fatigue and depressive symptoms in HD patients may be an interesting scope for future research.

In conclusion, our study suggests that endotoxemia and inflammation through IL-6 are related to fatigue in haemodialysis patients. These findings should help to generate additional longitudinal studies investigating the causative role of endotoxemia in the onset of chronic inflammation and fatigue in patients on chronic haemodialysis. Additional research is warranted to detect a common mediator for depression and fatigue that might become a potential target for therapeutic strategies. 


\section{References}

1. Kramer A, Pippias M, Noordzij M, et al. The European Renal Association - European Dialysis and Transplant Association (ERA-EDTA) Registry Annual Report 2015: a summary. Clin Kidney J. 2018;11(1):108-122.

2. United States Renal Data System. USRDS annual data report: Epidemiology of kidney disease in the United States. . 2018; https://www.usrds.org/2018/view/Default.aspx.

3. Davison SN, Jhangri GS. Impact of pain and symptom burden on the health-related quality of life of hemodialysis patients. J Pain Symptom Manage. 2010;39(3):477-485.

4. Horigan AE. Fatigue in hemodialysis patients: a review of current knowledge. J Pain Symptom Manage. 2012;44(5):715-724.

5. Hardy SE, Studenski SA. Qualities of fatigue and associated chronic conditions among older adults. J Pain Symptom Manage. 2010;39(6):1033-1042.

6. Jhamb M, Weisbord SD, Steel JL, Unruh M. Fatigue in patients receiving maintenance dialysis: a review of definitions, measures, and contributing factors. American journal of kidney diseases : the official journal of the National Kidney Foundation. 2008;52(2):353-365.

7. Artom M, Moss-Morris R, Caskey F, Chilcot J. Fatigue in advanced kidney disease. Kidney international. 2014;86(3):497-505.

8. Picariello F, Moss-Morris R, Macdougall IC, Chilcot AJ. The role of psychological factors in fatigue among end-stage kidney disease patients: a critical review. Clin Kidney J. 2017;10(1):79-88.

9. Brys AD, Lenaert B, Van Heugten CM, Gambaro G, Bossola M. Exploring the diurnal course of fatigue in patients on haemodialysis treatment and its relation with depressive symptoms and classical conditioning. J Pain Symptom Manage. 2019.

10. Saligan LN, Kim HS. A systematic review of the association between immunogenomic markers and cancer-related fatigue. Brain Behav Immun. 2012;26(6):830-848.

11. Flachenecker P, Bihler I, Weber F, Gottschalk M, Toyka KV, Rieckmann P. Cytokine mRNA expression in patients with multiple sclerosis and fatigue. Multiple sclerosis (Houndmills, Basingstoke, England). 2004;10(2):165-169.

12. Heesen C, Nawrath L, Reich C, Bauer N, Schulz KH, Gold SM. Fatigue in multiple sclerosis: an example of cytokine mediated sickness behaviour? Journal of neurology, neurosurgery, and psychiatry. 2006;77(1):34-39.

13. Moreland LW, Genovese MC, Sato R, Singh A. Effect of etanercept on fatigue in patients with recent or established rheumatoid arthritis. Arthritis and rheumatism. 2006;55(2):287-293.

14. Fung A, Vizcaychipi M, Lloyd D, Wan Y, Ma D. Central nervous system inflammation in disease related conditions: mechanistic prospects. Brain Res. 2012;1446:144-155.

15. Dantzer R. Cytokine-induced sickness behavior: mechanisms and implications. Annals of the New York Academy of Sciences. 2001;933:222-234.

16. Dantzer R, Kelley KW. Twenty years of research on cytokine-induced sickness behavior. Brain Behav Immun. 2007;21(2):153-160.

17. Karshikoff B, Sundelin T, Lasselin J. Role of Inflammation in Human Fatigue: Relevance of Multidimensional Assessments and Potential Neuronal Mechanisms. Frontiers in immunology. 2017;8:21.

18. Norheim KB, Jonsson G, Omdal R. Biological mechanisms of chronic fatigue. Rheumatology (Oxford, England). 2011;50(6):1009-1018.

19. Dantzer R, O'Connor JC, Freund GG, Johnson RW, Kelley KW. From inflammation to sickness and depression: when the immune system subjugates the brain. Nature reviews. Neuroscience. 2008;9(1):46-56. 
20. Howren MB, Lamkin DM, Suls J. Associations of depression with C-reactive protein, IL-1, and IL-6: a meta-analysis. Psychosomatic medicine. 2009;71(2):171-186.

21. Walker AK, Kavelaars A, Heijnen CJ, Dantzer R. Neuroinflammation and comorbidity of pain and depression. Pharmacological reviews. 2014;66(1):80-101.

22. Miller $\mathrm{AH}$, Raison $\mathrm{CL}$. The role of inflammation in depression: from evolutionary imperative to modern treatment target. Nature reviews. Immunology. 2016;16(1):22-34.

23. Yao $Q$, Lindholm B, Stenvinkel P. Inflammation as a cause of malnutrition, atherosclerotic cardiovascular disease, and poor outcome in hemodialysis patients. Hemodialysis international. International Symposium on Home Hemodialysis. 2004;8(2):118-129.

24. Kaysen GA. The microinflammatory state in uremia: causes and potential consequences. Journal of the American Society of Nephrology : JASN. 2001;12(7):1549-1557.

25. Bambauer R, Schmidt R, Falkenhagen D, Walther J, Jung WK. Sterile and endotoxin free dialysis fluid for hemodialysis. Biomaterials, artificial cells, and immobilization biotechnology : official journal of the International Society for Artificial Cells and Immobilization Biotechnology. 1991;19(1):71-83.

26. Wong J, Vilar E, Farrington K. Endotoxemia in end-stage kidney disease. Seminars in dialysis. 2015;28(1):59-67.

27. Benson S, Engler H, Wegner A, et al. What Makes You Feel Sick After Inflammation? Predictors of Acute and Persisting Physical Sickness Symptoms Induced by Experimental Endotoxemia. Clinical pharmacology and therapeutics. 2017;102(1):141-151.

28. Coulliette AD, Arduino MJ. Hemodialysis and water quality. Seminars in dialysis. 2013;26(4):427-438.

29. Vanholder R, Ringoir S, Dhondt A, Hakim R. Phagocytosis in uremic and hemodialysis patients: a prospective and cross sectional study. Kidney international. 1991;39(2):320-327.

30. Cavaillon JM, Poignet JL, Fitting C, Delons S. Serum interleukin-6 in long-term hemodialyzed patients. Nephron. 1992;60(3):307-313.

31. Pereira BJ, Shapiro L, King AJ, Falagas ME, Strom JA, Dinarello CA. Plasma levels of IL-1 beta, TNF alpha and their specific inhibitors in undialyzed chronic renal failure, CAPD and hemodialysis patients. Kidney international. 1994;45(3):890-896.

32. Bossola M, Sanguinetti M, Scribano D, et al. Circulating bacterial-derived DNA fragments and markers of inflammation in chronic hemodialysis patients. Clinical journal of the American Society of Nephrology : CJASN. 2009;4(2):379-385.

33. El-Koraie AF, Naga YS, Saaran AM, Farahat NG, Hazzah WA. Endotoxins and inflammation in hemodialysis patients. Hemodialysis international. International Symposium on Home Hemodialysis. 2013;17(3):359-365.

34. Terawaki H, Yokoyama K, Yamada Y, et al. Low-grade endotoxemia contributes to chronic inflammation in hemodialysis patients: examination with a novel lipopolysaccharide detection method. Therapeutic apheresis and dialysis : official peer-reviewed journal of the International Society for Apheresis, the Japanese Society for Apheresis, the Japanese Society for Dialysis Therapy. 2010;14(5):477-482.

35. Bossola M, Di Stasio E, Giungi S, Rosa F, Tazza L. Fatigue is associated with serum interleukin-6 levels and symptoms of depression in patients on chronic hemodialysis. J Pain Symptom Manage. 2015;49(3):578-585.

36. Bossola M, Luciani G, Giungi S, Tazza L. Anorexia, fatigue, and plasma interleukin-6 levels in chronic hemodialysis patients. Renal failure. 2010;32(9):1049-1054.

37. Bossola M, Luciani G, Tazza L. Fatigue and its correlates in chronic hemodialysis patients. Blood purification. 2009;28(3):245-252.

38. Bossola M, Di Stasio E, Sanguinetti M, et al. Serum Endotoxin Activity Measured with Endotoxin Activity Assay Is Associated with Serum Interleukin-6 Levels in Patients on Chronic Hemodialysis. Blood purification. 2016;42(4):294-300. 
39. Ware JE, Snow KK, Kosinski M, Gandek B. SF-36 Health Survey: Manual and Interpretation Guide. Boston: The Health Institute, New England Medical Center 1993.

40. Ju A, Unruh M, Davison S, et al. Establishing a Core Outcome Measure for Fatigue in Patients on Hemodialysis: A Standardized Outcomes in Nephrology-Hemodialysis (SONG-HD) Consensus Workshop Report. American journal of kidney diseases : the official journal of the National Kidney Foundation. 2018;72(1):104-112.

41. Ju A, Unruh ML, Davison SN, et al. Patient-Reported Outcome Measures for Fatigue in Patients on Hemodialysis: A Systematic Review. American journal of kidney diseases : the official journal of the National Kidney Foundation. 2018;71(3):327-343.

42. Galeoto G, Sansoni J, Scuccimarri M, et al. A Psychometric Properties Evaluation of the Italian Version of the Geriatric Depression Scale. Depression research and treatment. 2018;2018:1797536.

43. Romaschin AD, Klein DJ, Marshall JC. Bench-to-bedside review: Clinical experience with the endotoxin activity assay. Critical care (London, England). 2012;16(6):248.

44. Di lorio B, Cillo N, Cirillo M, De Santo NG. Charlson Comorbidity Index is a predictor of outcomes in incident hemodialysis patients and correlates with phase angle and hospitalization. The International journal of artificial organs. 2004;27(4):330-336.

45. Katz S, Ford AB, Moskowitz RW, Jackson BA, Jaffe MW. STUDIES OF ILLNESS IN THE AGED. THE INDEX OF ADL: A STANDARDIZED MEASURE OF BIOLOGICAL AND PSYCHOSOCIAL FUNCTION. Jama. 1963;185:914-919.

46. Boulware LE, Liu Y, Fink NE, et al. Temporal relation among depression symptoms, cardiovascular disease events, and mortality in end-stage renal disease: contribution of reverse causality. Clinical journal of the American Society of Nephrology : CJASN. 2006;1(3):496-504.

47. Cilan H, Oguzhan N, Unal A, et al. Relationship between depression and proinflammatory cytokine levels in hemodialysis patients. Renal failure. 2012;34(3):275-278.

48. Dervisoglu E, Kir HM, Kalender B, Eraldemir C, Caglayan C. Depressive symptoms and proinflammatory cytokine levels in chronic renal failure patients. Nephron. Clinical practice. 2008;108(4):c272-277.

49. Sonikian M, Metaxaki P, Papavasileiou D, et al. Effects of interleukin-6 on depression risk in dialysis patients. American journal of nephrology. 2010;31(4):303-308.

50. Taraz M, Khatami MR, Gharekhani A, Abdollahi A, Khalili H, Dashti-Khavidaki S. Relationship between a pro- and anti-inflammatory cytokine imbalance and depression in haemodialysis patients. European cytokine network. 2012;23(4):179-186.

51. Hung KC, Wu CC, Chen HS, et al. Serum IL-6, albumin and co-morbidities are closely correlated with symptoms of depression in patients on maintenance haemodialysis. Nephrology, dialysis, transplantation : official publication of the European Dialysis and Transplant Association - European Renal Association. 2011;26(2):658-664. 



\section{Chapter 3}

Peridialytic serum cytokine levels and their relationship with postdialysis fatigue and recovery in patients on chronic haemodialysis - A preliminary

study

Brys ADH, Di Stasio E, Lenaert B, Picca A, Calvani R, Marzetti E, Gambaro G, Bossola M

Published in Cytokine. 2020;135:155223 


\section{Abstract}

\section{Background}

The aetiology of postdialysis fatigue (PDF), an intermittent but debilitating fatigue occurring after haemodialysis (HD) treatment, is still unclear. In other inflammatory diseases, increasing evidence points towards the involvement of inflammation in the onset of fatigue symptoms. Altered serum levels of inflammatory cytokines have also been shown in HD patients. Therefore, we investigated whether pre- and postdialysis serum levels of pro- and anti-inflammatory cytokines (i.e. IL-1 $\beta$, IL-6, TNF- $\alpha$ and IL-10) or their intradialytic changes (if any) were related to PDF or the time HD patients reported needing to recover from HD treatment (TIRD).

\section{Methods}

Serum levels of IL-1 $\beta$, IL- 6 , TNF- $\alpha$ and IL-10 were measured immediately before and after HD in 45 patients using commercially available kits on an ELLA ${ }^{\text {TM }}$ automated immunoassay system. The presence and severity of PDF as well as TIRD duration were assessed by self-report measures.

\section{Key results}

Seventy-four percent of patients reported PDF, with a median PDF severity index of 3.30 [IQR: 3.00-4.30] on a scale from 1 to 5. Median TIRD was 120 min [IQR: 60-480]. PDF severity correlated strongly with TIRD, $r_{s}=0.85, p<0.001$. Only predialysis levels of IL-10 significantly and positively correlated with PDF severity $\left(r_{\mathrm{s}}=0.43, p=0.003\right)$.

\section{Conclusion}

Findings of the present study do not support the involvement of inflammation in the onset of PDF or the time patients needed to recover from HD treatment. A positive, but counterintuitive relationship was found between predialysis levels of antiinflammatory IL-10 and PDF severity, which warrants further research. 


\section{Introduction}

Worldwide, approximately three million people receive renal replacement therapy (RRT) because of end-stage kidney disease (ESKD). ${ }^{1}$ With an estimated prevalence of 465 and 1437 per million people in Europe and the U.S. respectively, haemodialysis (HD) is by far the most common form of RRT. ${ }^{2-4}$

Twenty to $80 \%$ of HD patients experience intermittent fatigue after their regular HD treatment and need to rest or sleep in order to recover. ${ }^{5}$ This condition is conventionally termed 'postdialysis fatigue' (PDF) and has been described as feeling worn out, drained or exhausted as a result of HD treatment. ${ }^{6}$ PDF is one of the most debilitating symptoms among HD patients and reduces their health-related quality of life (HrQoL). ${ }^{7}$ However, as an inherently subjective experience, PDF is difficult to define and guidelines to investigate PDF are lacking. The most common way to assess PDF is that proposed by Sklar et al., pioneers in PDF studies, asking HD patients an open-ended question: "Do you feel better or worse after dialysis? If worse, please specify in which way(s)".$^{6-8}$ Patients spontaneously mentioning fatigue are considered to suffer from PDF and receive additional questions regarding its intensity, frequency and duration. ${ }^{9}$ Another frequently adopted approach is that of the London Daily/Nocturnal Hemodialysis Study, which evaluated HrQoL outcomes in HD patients. ${ }^{10}$ In this study, patients were asked how much time they needed to recover from a dialysis session (TIRD). ${ }^{10}$ Although PDF and TIRD are not synonymous, TIRD may be used as an indirect measure of PDF. ${ }^{5}$ Further, a number of studies sought to clarify mechanisms underlying PDF by investigating its relationship with demographical, clinical, laboratory or dialysis-related variables, such as a rapid osmolar flux, excessive ultrafiltration or bioincompatible dialysis membranes. ${ }^{6-9,11,12}$ However, findings remain conflicting and no causal relationship has yet been demonstrated. ${ }^{5}$

Interestingly, in other illnesses such as infectious diseases, cancer, neurodegenerative diseases (e.g. multiple sclerosis) and auto-immune diseases (e.g. rheumatoid arthritis) there is mounting evidence for the involvement of inflammation in the development of related fatigue. ${ }^{13-17}$ For instance, pro-inflammatory cytokines, such as interleukin$1 \beta$ (IL-1 $\beta)$, IL- 6 and tumour necrosis factor $\alpha$ (TNF- $\alpha$ ), may mediate a constellation of symptoms, often referred to as 'sickness behaviour', by interfering with the central nervous system. ${ }^{18}$ This 'sickness behaviour' may include fatigue and decreased 
physical activity, reduced ability to concentrate and an altered mood state, withdrawal from social interaction and loss of appetite. ${ }^{19,20}$ It may be seen as a biologically triggered coping mechanism by inducing rest in order to conserve energy and thus allow recovery to take place. ${ }^{21}$ In contrast, anti-inflammatory cytokines, such as IL-10, regulate the intensity and duration of sickness behaviour by inhibiting the production of pro-inflammatory cytokines and attenuating their signaling. ${ }^{22}$

Approximately $30-50 \%$ of HD patients show evidence of an inflammatory response. ${ }^{23}$ Both ESKD and dialysis-related factors may promote the inflammatory status commonly observed in this patient population. First, accumulation of proinflammatory components, reduction of plasma antioxidant activity and increased oxidative stress are attributable to renal failure itself. ${ }^{24,25}$ In addition, the intestinal barrier may be disrupted in HD patients by the uremic environment, causing gut microbiota translocation. ${ }^{26}$ Furthermore, due to impaired humoral and cellular immunity, vascular accesses and often co-occurring diabetes mellitus, HD patients are more susceptible to infections. ${ }^{27}$ Moreover, during the HD procedure, activation of circulating mononuclear cells, which stimulate cytokine production, may occur when blood interacts with bioincompatible dialysis membranes. Finally, microbial or endotoxin contaminated dialysis water, may further induce transmembrane activation of circulating immune cells. ${ }^{24,25}$

To date, a number of studies showed that higher scores of overall fatigue experience in HD patients were associated with higher levels of serum IL-6, suggesting this cytokine may be involved in modulating a general sense of fatigue in HD patients. ${ }^{28-31}$ However, the relationship between PDF or TIRD on the one hand and peridialytic (preand postdialysis) cytokine levels or their intradialytic changes (if any) on the other hand, has been scarcely investigated. Only two studies investigated the relationship between PDF and peridialytic levels of TNF- $\alpha .{ }^{32,33}$ However, their findings are conflicting as the first study found that the intradialytic rise in TNF- $\alpha$ concentrations was related to PDF, whereas the second report did not show any association between cytokine rise and PDF symptoms.

Therefore, the present study aimed to advance our understanding of PDF by investigating whether pre- and postdialysis serum levels of IL-1 $\beta, I L-6, I L-10$, and TNF- $\alpha$ or their changes were related to PDF or TIRD in a sample of well-characterized HD patients. 


\section{Subjects and methods}

\section{Study sample and design}

Between January and December 2017, all patients undergoing chronic HD treatment for at least one year at the haemodialysis unit of the Fondazione Policlinico Universitario A. Gemelli IRCCS (Rome, Italy) were considered eligible for this observational study. Exclusion criteria were: $<18$ years of age, acute or chronic infections, antibiotic therapy in the previous two weeks, diagnosed auto-immune disorders, neurodegenerative diseases, cancer, and dementia. The study was carried out in accordance with the Declaration of Helsinki and the study protocol was approved by the ethics committee of the Università Cattolica del Sacro Cuore (Rome, Italy) (0022625). A written informed consent was obtained from all patients prior to inclusion.

\section{Data collection}

\section{Blood collection}

Blood samples were collected under sterile conditions from either the arteriovenous fistula or the central venous catheter prior to and immediately after the scheduled HD session at the beginning of the week. One tube was delivered to the centralized diagnostic laboratory of the Fondazione Policlinico Universitario "Agostino Gemelli" IRCCS for standard biochemistry analysis. Briefly, plasma was separated and collected within 30 minutes and stored at $-70^{\circ} \mathrm{C}$ if not analyzed immediately. Standard laboratory parameters were measured within four hours of specimen collection using Advia2120i instruments (Advia Centaur XPT, Siemens Healthineers, Munich, Germany) according to the manufacturer's instructions. The remaining blood tubes were processed for serum separation in the Biogerontology lab of the Università Cattolica del Sacro Cuore. After 30 min of blood clotting at room temperature, samples were centrifuged at $1000 \times \mathrm{g}$ for $15 \mathrm{~min}$ at $4^{\circ} \mathrm{C}$. The upper clear fraction (serum) was recovered in $0.5-\mathrm{ml}$ aliquots and stored at $-80^{\circ} \mathrm{C}$ until analysis. The levels of serum IL-1 $\beta$, IL-6, IL-10 and TNF- $\alpha$ were measured using a $32 \times 4$ multiplex custom-designed plate on an ELLA ${ }^{\mathrm{TM}}$ automated immunoassay system (Bio-Techne, San Jose, CA, USA) according to the manufacturer's instructions. 
Assessment of postdialysis fatigue and time to recover after haemodialysis

PDF was assessed according to the recommendations of Sklar et al. ${ }^{6}$, i.e. patients who spontaneously answered to be fatigued after their haemodialysis sessions when asked the open-ended question: "Do you feel worse after dialysis? If so, please specify which way(s)", were considered to suffer from PDF. Patients experiencing PDF were additionally requested to rate the intensity, duration and frequency of PDF on a scale from 1 (low) to 5 (high). The average of these three parameters was used as a measure for the experienced PDF severity (i.e. a PDF severity index).

TIRD was assessed following the approach of the London Daily/Nocturnal Hemodialysis Study. ${ }^{34}$ Patients were asked to answer the open-ended question: "How long does it take you to recover from a dialysis session?". Answers were converted into total number of minutes. The London Daily/Nocturnal Hemodialysis Study previously reported that this simple question was easily interpreted by HD patients, elicited a clear response, had excellent test-retest reliability $(r=0.95, p<0.001)$, and was significantly associated with fatigue scores at the end of HD treatment $(r=0.51, p<0.001) .^{34}$

\section{Baseline and clinical data}

Demographical and clinical data were obtained from medical records at the hospital at the time of inclusion in the study.

\section{Haemodialysis treatment}

Patients received bicarbonate HD treatment for four hours, three times weekly. The blood flow ranged from 250 to $300 \mathrm{ml} / \mathrm{min}$ with a dialysis rate flow of $500 \mathrm{ml} / \mathrm{min}$. Biocompatible, high permeability membranes were used.

\section{Procedure}

After patients gave their informed consent, blood samples were provided during a regularly scheduled HD session at the beginning of the week. In addition, patients were interviewed by the attending physician within the same week and requested to answer the aforementioned PDF and TIRD questions. 


\section{Statistical analyses}

Statistical analyses were performed using the SPSS software, version 25 (IBM Corp., Armonk, NY, USA). The Shapiro-Wilk test indicated that the assumption of normality of distribution was violated for all variables of interest (i.e. pre- and postdialysis cytokine levels, TIRD, and PDF severity index). Therefore, non-parametric tests were used. Data of continuous and categorical variables are reported as mean \pm SD or median [range] and frequencies, respectively. For analyses involving multiple comparisons, a $p$-value of $<0.01$ was considered statistically significant. Otherwise statistical significance was set at $p<0.05$.

To investigate differences in cytokine levels pre- and postdialysis, the Wilcoxon signed rank test was used. To assess whether pre- and postdialysis cytokine levels or their intradialytic changes (delta) were related to PDF severity or TIRD, Spearman's rho correlation coefficients were calculated. For this analysis, patients without PDF symptoms were considered having a PDF severity index of zero. The Mann-Whitney $U$ test was used to test whether demographical, clinical and standard laboratory variables, as well as pre- and postdialysis cytokine levels or their delta varied between patients suffering from PDF and patients without PDF symptoms. The same analysis was run to investigate differences in cytokine levels between patients with a short TIRD ( $\leq$ the median TIRD of the study sample) and a long TIRD ( $>$ the median TIRD of the study sample). The Pearson $\mathrm{Chi}^{2} /$ Fisher exact test was used to assess the interrelationship between patients with or without PDF symptoms and patients with a shorter or longer TIRD, with the median TIRD used as the cut-off value.

\section{Results}

\section{Sample characteristics}

Forty-five HD patients were enrolled in the study (29 men, 64\%). Twelve patients (26\%) did not experience PDF. Thirty-three patients (74\%) reported to suffer from PDF, with a median PDF severity index of 3.30 [IQR: 3.00-4.30]. The median TIRD was $120 \mathrm{~min}$ [IQR: 60-480]. Twenty-one patients (47\%) reported a TIRD >120 min. Demographical, clinical and standard laboratory characteristics, as well as their average values in individuals with and without PDF are reported in Table 3.1. 


\section{Pre- and postdialysis cytokine levels}

Postdialysis serum levels of IL-1 $\beta$ ( $M d n=0.19)$ were significantly lower than those measured predialysis $(M d n=0.22), W_{+}=262.5, z=-2.71, p=0.007$, with a small effect size of $r=0.29$. IL-10 serum concentrations postdialysis $(M d n=4.81)$ was significantly higher than in predialysis $(M d n=3.60), W_{+}=968.5, z=5.09, p<0.001$, with a large effect size of $r=0.54$. In contrast, IL- 6 postdialysis ( $M d n=7.52$ ) was not significantly different from IL-6 predialysis $(M d n=7.94), W_{+}=394, z=-1.36, p=0.174$, with a small effect size of $r=0.14$. Likewise, TNF- $\alpha$ postdialysis $(M d n=19.24)$ did not vary significantly from its predialysis levels $(M d n=18.29), W_{+}=558.5, z=0.46, p=0.644$, with a small effect size of $r=0.07$.

\section{Relationship between cytokine levels and PDF and TIRD}

Spearman's rho correlation coefficients for pre- and postdialysis cytokine levels and their delta on the one hand and patients' reported PDF severity and needed time of recovery after $\mathrm{HD}$ on the other hand, are displayed in Table 3.2. Only IL-10 levels before dialysis were significantly related to PDF severity $\left(r_{s}=0.43, p=0.003\right)$. Subsequent analyses with regard to intensity, duration, and frequency of PDF revealed that predialysis IL-10 levels correlated significantly with the intensity and frequency of PDF symptoms $\left(r_{s}=0.44, p=0.002 ; r_{s}=0.45, p=0.002\right.$, respectively) and only moderately though not significantly with the PDF duration $\left(r_{s}=0.36, p=0.016\right)$. No statistically significant association was found between postdialysis cytokine levels or cytokine delta and PDF severity or TIRD. Due to our limited sample size, an exploratory multiple regression analysis was run only with variables where MannWhitney $U$ tests comparing individuals with and without PDF revealed statistical significance at $p<0.05$ in Table 3.1 as predictors (i.e. predialysis levels of IL-10, dialysis vintage and serum sodium levels). Results showed that none of these variables significantly predicted PDF severity. Furthermore, PDF severity correlated strongly with TIRD, $r_{s}=0.85,95 \% \mathrm{Bca} \mathrm{Cl}[0.72,0.92], p<0.001$. 
Table 3.1 Participants' demographical, clinical and laboratory characteristics.

\begin{tabular}{|c|c|c|c|c|}
\hline \multirow[t]{2}{*}{ Characteristics } & \multirow{2}{*}{$\begin{array}{l}\text { All patients ( } n=45 \text { ) } \\
\text { Mean } \pm \text { SD [range] / } \\
n \text { (percentage) }\end{array}$} & \multirow{2}{*}{$\begin{array}{l}\text { No PDF }(n=12) \\
\text { Mean } \pm \text { SD [range] / } \\
n \text { (percentage) }\end{array}$} & \multirow{2}{*}{$\begin{array}{c}\text { PDF }(\mathbf{n = 3 3 )} \\
\text { Mean } \pm \text { SD [range] / } \\
n \text { (percentage) }\end{array}$} & \multirow[t]{2}{*}{$p$} \\
\hline & & & & \\
\hline \multicolumn{5}{|l|}{ Demographical characteristics } \\
\hline Age (years) & $63 \pm 17[21-91]$ & $68 \pm 14[38-91]$ & $61 \pm 18[21-88]$ & 0.304 \\
\hline Sex (men) & $29(64 \%)$ & $10(83 \%)$ & $19(58 \%)$ & 0.164 \\
\hline \multicolumn{5}{|l|}{ Clinical characteristics } \\
\hline \multicolumn{5}{|l|}{ Primary cause of ESRD } \\
\hline hypertension & $12(27 \%)$ & $4(33 \%)$ & $8(24 \%)$ & 0.705 \\
\hline glomerulonephritis & $8(18 \%)$ & $2(17 \%)$ & $6(18 \%)$ & 1.000 \\
\hline diabetes & $10(22 \%)$ & $4(33 \%)$ & $6(18 \%)$ & 0.418 \\
\hline interstitial nephritis & $2(4 \%)$ & $1(8 \%)$ & $1(3 \%)$ & 0.467 \\
\hline polycystic kidney disease & $5(11 \%)$ & $0(0 \%)$ & $5(15 \%)$ & 0.303 \\
\hline others/unknown & $8(18 \%)$ & $1(8 \%)$ & $7(21 \%)$ & 0.419 \\
\hline Dialysis vintage (months) & $69 \pm 74[7-936]$ & $46 \pm 65[10-216]$ & $77 \pm 76[7-336]$ & 0.039 \\
\hline \multirow[t]{2}{*}{ Body Mass Index $\left(\mathrm{kg} / \mathrm{m}^{2}\right)$} & $24.4 \pm 4.8$ & $23.4 \pm 3.4$ & $24.7 \pm 5.2$ & 0.719 \\
\hline & {$[17.1-39.2]$} & [19.6-31.4] & [17.1-39.2] & \\
\hline Charlson Comorbidity Index & $1.7 \pm 1.5[0-6]$ & $2.2 \pm 1.7[0-5]$ & $1.5 \pm 1.4[0-6]$ & 0.129 \\
\hline \multicolumn{5}{|l|}{ Laboratory characteristics } \\
\hline$K t / V$ urea clearance & $1.4 \pm 0.3$ & $1.2 \pm 0.5$ & $1.5 \pm 0.2$ & 0.148 \\
\hline Serum urea (mg/dl) & $72 \pm 17$ & $72 \pm 21$ & $72 \pm 15$ & 0.846 \\
\hline Serum creatinine (mg/dl) & $9.3 \pm 2.7$ & $7.8 \pm 1.8$ & $9.9 \pm 2.7$ & 0.085 \\
\hline Haemoglobin (g/dl) & $10.8 \pm 0.9$ & $10.8 \pm 0.6$ & $10.7 \pm 1.0$ & 0.867 \\
\hline Serum albumin (mg/dl) & $36 \pm 3$ & $35 \pm 3$ & $36 \pm 3$ & 0.290 \\
\hline Serum Sodium (mmol/l) & $139 \pm 3$ & $140 \pm 3$ & $138 \pm 3$ & 0.023 \\
\hline Dialysate Sodium (mmol/l) & $139 \pm 2$ & $139 \pm 1$ & $139 \pm 2$ & 0.938 \\
\hline Calcium (mg/dl) & $9.3 \pm 0.8$ & $9.1 \pm 1.2$ & $9.4 \pm 0.6$ & 0.911 \\
\hline Phosphorus (mg/dl) & $4.7 \pm 1.5$ & $4.5 \pm 1.2$ & $4.8 \pm 1.6$ & 0.504 \\
\hline $\mathrm{CRP}(\mathrm{mg} / \mathrm{ml})$ & $4.5 \pm 5.2$ & $3.0 \pm 4.3$ & $5.2 \pm 5.6$ & 0.301 \\
\hline \multicolumn{5}{|l|}{$\mathrm{IL}-1 \beta(\mathrm{pg} / \mathrm{ml})$ (median [IQR]) } \\
\hline Predialysis & $0.22[0.18-0.33]$ & $0.22[0.14-0.28]$ & $0.22[0.17-0.36]$ & 0.505 \\
\hline Postdialysis & 0.19 [0.12-0.27] & $0.16[0.05-0.25]$ & 0.19 [0.12-0.29] & 0.186 \\
\hline \multicolumn{5}{|l|}{ IL-6 (pg/ml) (median [IQR]) } \\
\hline Predialysis & $7.9[5.2-11.0]$ & $7.2[5.4-9.5]$ & 7.9 [4.6-13.2] & 0.719 \\
\hline Postdialysis & $7.5[4.6-11.6]$ & $6.6[3.6-9.8]$ & $7.5[4.5-12.2]$ & 0.538 \\
\hline \multicolumn{5}{|l|}{ TNF- $\alpha(p g / m l)$ (median [IQR]) } \\
\hline Predialysis & $18.3[16.6-21.3]$ & $16.4[15.4-18.8]$ & $18.8[16.6-22.3]$ & 0.124 \\
\hline Postdialysis & $19.2[15.9-21.7]$ & $18.7[14.9-21.2]$ & $19.6[15.7-22.2]$ & 0.642 \\
\hline \multicolumn{5}{|l|}{ IL-10 (pg/ml) (median [IQR]) } \\
\hline Predialysis & $3.6[3.0-4.4]$ & $3.0[2.6-3.5]$ & $4.1[3.3-4.6]$ & 0.013 \\
\hline Postdialysis & $4.8[3.7-6.5]$ & $3.8[2.7-5.6]$ & $5.1[3.8-7.1]$ & 0.143 \\
\hline \multicolumn{5}{|l|}{ Interview questions } \\
\hline Postdialysis fatigue (PDF) & $33(74 \%)$ & - & - & - \\
\hline PDF severity index (median [IQR]) & $3.3[3.0-4.3]$ & - & $3.3[3.0-4.3]$ & - \\
\hline Time of Recovery after & & & & \\
\hline $\begin{array}{l}\text { Dialysis (TIRD) (minutes) } \\
\text { (median [IQR]) }\end{array}$ & $120[60-480]$ & 30 [10-105] & $240[60-720]$ & $<0.001$ \\
\hline
\end{tabular}

CRP: C-reactive protein, IL: interleukin, TNF- $\alpha$ : tumour necrosis factor $\alpha$, IQR: interquartile range, PDF: postdialysis fatigue, $p$ : $p$-value 
Table 3.2 Univariate correlation analysis between peridialytic cytokine levels and their intradialytic changes (delta) on the one hand and postdialysis fatigue (PDF) severity and time of recovery after haemodialysis (TIRD) on the other hand (Spearman's rho correlation coefficients [95\% Bca Cl]) (N=45).

\begin{tabular}{lcccc}
\hline Cytokine levels & PDF severity & $\boldsymbol{p}$-value & TIRD & $\boldsymbol{p}$-value \\
\hline Before dialysis & & & & \\
IL-1 $\beta$ & $-0.04[-0.38,0.27]$ & 0.802 & $-0.03[-0.35,0.28]$ & 0.824 \\
IL-6 & $0.10[-0.18,0.36]$ & 0.526 & $0.03[-0.23,0.29]$ & 0.864 \\
TNF- $\alpha$ & $0.15[-0.11,0.37]$ & 0.342 & $0.03[-0.25,0.28]$ & 0.850 \\
IL-10 & $0.43[0.13,0.68]$ & 0.003 & $0.25[-0.06,0.54]$ & 0.095 \\
After dialysis & & & & \\
IL-1 $\beta$ & $0.12[-0.20,0.40]$ & 0.417 & $0.07[-0.27,0.38]$ & 0.661 \\
IL-6 & $0.14[-0.14,0.41]$ & 0.373 & $0.11[-0.20,0.39]$ & 0.473 \\
TNF- $\alpha$ & $0.08[-0.20,0.37]$ & 0.626 & $-0.09[-0.36,0.20]$ & 0.538 \\
IL-10 & $0.27[-0.07,0.57]$ & 0.072 & $0.12[-0.20,0.45]$ & 0.430 \\
Delta & & & & \\
IL-1 $\beta$ & $0.19[-0.07,0.43]$ & 0.221 & $0.13[-0.15,0.38]$ & 0.414 \\
IL-6 & $-0.12[-0.40,0.19]$ & 0.441 & $-0.03[-0.32,0.28]$ & 0.857 \\
TNF- $\alpha$ & $-0.05[-0.32,0.25]$ & 0.737 & $-0.14[-0.40,0.18]$ & 0.373 \\
IL-10 & $0.02[-0.28,0.31]$ & 0.909 & $-0.08[-0.37,0.22]$ & 0.589 \\
\hline
\end{tabular}

Tables 3.3 and 3.4 show pre-and postdialysis cytokine levels and their delta according to the presence or absence of PDF symptoms and TIRD below or equal to the median or above the median of $120 \mathrm{~min}$, respectively. Neither pre- or postdialysis levels of IL$1 \beta$, IL-6, IL-10 and TNF- $\alpha$, nor their delta significantly varied between patients with or without PDF symptoms. Similarly, IL-1 $\beta$, IL-6, IL-10 and TNF- $\alpha$ levels before and after dialysis or their delta did not significantly vary between patients with a TIRD $\leq 120 \mathrm{~min}$ or $>120 \mathrm{~min}$.

Table 3.3 Pro- and anti-inflammatory cytokine levels pre-and postdialysis and their intradialytic changes (delta) according to the presence or absence of postdialysis fatigue (PDF) ( $N=45$ ).

\begin{tabular}{lccc}
\hline Cytokine levels & $\begin{array}{c}\text { No PDF }(\mathbf{n = 1 2}) \\
\text { Median [IQR] }\end{array}$ & $\begin{array}{c}\text { PDF (n=33) } \\
\text { Median [IQR] }\end{array}$ & $p$-value \\
\hline Before dialysis & & & \\
IL-1 $\beta$ & $0.22[0.18,0.27]$ & $0.22[0.18,0.35]$ & 0.518 \\
IL-6 & $7.20[5.68,8.88]$ & $7.94[4.71,13.00]$ & 0.732 \\
TNF- $\alpha$ & $17.38[16.17,18.28]$ & $18.75[16.70,21.86]$ & 0.128 \\
IL-10 & $2.99[2.59,3.45]$ & $4.06[3.37,4.59]$ & 0.013 \\
After dialysis & & & \\
IL-1 & $0.16[0.06,0.21]$ & $0.19[0.13,0.29]$ & 0.189 \\
IL-6 & $6.63[4.37,8.82]$ & $7.51[4.56,11.88]$ & 0.551 \\
TNF- $\alpha$ & $18.74[15.59,20.02]$ & $19.59[15.85,22.20]$ & 0.354 \\
IL-10 & $3.83[2.88,5.15]$ & $5.11[3.89,7.03]$ & 0.149 \\
Delta & & & \\
IL-1 $\beta$ & $-0.06[-0.09,-0.04]$ & $-0.03[-0.12,0.03]$ & 0.354 \\
IL-6 & $-0.90[-1.27,0.86]$ & $-0.60[-1.78,0.68]$ & 0.731 \\
TNF- $\alpha$ & $0.73[-3.18,2.25]$ & $0.14[-2.18,3.91]$ & 0.979 \\
IL-10 & $0.86[0.52,1.63]$ & $0.81[0.40,1.81]$ & 0.970 \\
\hline
\end{tabular}


Table 3.4 Pro- and anti-inflammatory parameters concentrations pre- and postdialysis and their intradialytic changes (delta) according to the time needed for recovery after haemodialysis (TIRD) ( $\mathrm{N}=45)$.

\begin{tabular}{lccc}
\hline Cytokine levels & $\begin{array}{c}\text { TIRD } \leq \mathbf{1 2 0} \text { min }(\mathbf{n}=\mathbf{2 4}) \\
\text { Median [IQR] }\end{array}$ & $\begin{array}{c}\text { TIRD }>\mathbf{1 2 0} \text { min }(\mathbf{n}=\mathbf{2 1}) \\
\text { Median [IQR] }\end{array}$ & $\boldsymbol{p}$-value \\
\hline Before dialysis & & & \\
IL-1 $\beta$ & $0.22[0.19,0.34]$ & $0.27[0.15,0.31]$ & 0.982 \\
IL-6 & $7.69[5.41,9.65]$ & $7.94[4.71,15.22]$ & 0.617 \\
TNF- $\alpha$ & $17.99[16.66,21.01]$ & $18.65[16.55,21.27]$ & 0.991 \\
IL-10 & $3.40[2.81,4.11]$ & $4.22[3.48,4.98]$ & 0.043 \\
After dialysis & & & \\
IL-1 $\beta$ & $0.19[0.11,0.23]$ & $0.18[0.13,0.30]$ & 0.617 \\
IL-6 & $7.36[4.39,10.61]$ & $7.64[4.86,13.65]$ & 0.387 \\
TNF- $\alpha$ & $19.29[15.63,21.01]$ & $19.24[15.85,22.20]$ & 0.891 \\
IL-10 & $4.37[3.29,5.48]$ & $5.21[4.01,7.03]$ & 0.295 \\
Delta & & & \\
IL-1 $\beta$ & $-0.06[-0.12,-0.02]$ & $-0.02[-0.12,0.06]$ & 0.446 \\
IL-6 & $-0.66[-1.55,0.72]$ & $-0.61[-1.78,0.68]$ & 0.820 \\
TNF- $\alpha$ & $0.27[-3.23,4.59]$ & $0.27[-1.82,3.61]$ & 0.785 \\
IL-10 & $1.03[0.50,1.72]$ & $0.72[0.36,2.19]$ & 0.716 \\
\hline
\end{tabular}

Finally, a significant association was found between a long versus short TIRD (cut-off at $120 \mathrm{~min}$ ) and whether patients reported to suffer from PDF or not, $\chi^{2}(1)=5.92$, $p=0.020$, Cramers' $V=0.36$, odds ratio $=6.78$. The odds ratio shows that the likelihood of patients suffering from PDF were 6.78 times higher if they had a TIRD>120 min.

\section{Discussion}

Previous studies in chronic inflammatory diseases demonstrated that in response to peripherally produced pro-inflammatory cytokines, such as IL-1 $\beta$, IL- 6 and TNF- $\alpha$, the central nervous system may induce behavioural changes, including fatigue. ${ }^{17,35}$ Recently, positive associations between serum IL-6 levels and fatigue symptoms in general have been reported in HD patients as well. ${ }^{29}$ The present study was conducted to assess whether fatigue symptoms specifically occurring after haemodialysis or the time patients needed to recover from a regular HD treatment would be related to preor postdialysis levels of pro- and anti-inflammatory cytokines (IL-1 3, IL-6, TNF- $\alpha$ and IL-10).

First, our results showed that pro-inflammatory cytokine levels of IL- 6 and TNF- $\alpha$ did not significantly change over the HD session. In contrast, levels of IL-1 $\beta$ decreased 
significantly after HD, although the effect size was small. Moreover, the antiinflammatory cytokine IL-10 was significantly, though moderately, higher before dialysis than afterwards. These findings are in contrast with the hypothesis of a potentially increased inflammatory response during HD. The possibility that these cytokines (with a molecular weight $>15 \mathrm{kDa}$ each) may have been cleared during the HD procedure should be discarded. Indeed, modern high-flux dialysis membranes are especially effective at removing small molecules, but clearance of molecules heavier than $15 \mathrm{kDa}$ is poor. ${ }^{36}$ However, once released, most cytokines have relatively short half-lives (from minutes to a couple of hours). ${ }^{37}$ Therefore, transient, dialysis-related rises in cytokine levels may not have been detected by only taking blood samples at a four-hour interval.

Second, in this HD population no significant relationship was found between peridialytic cytokine levels and the presence or absence of PDF and a short or long TIRD, respectively. Neither postdialysis cytokine levels of IL-1 $\beta, I L-6, I L-10$ and TNF- $\alpha$ nor their intradialytic change scores correlated significantly with PDF severity or TIRD. Only predialysis levels of the anti-inflammatory IL-10 significantly and positively correlated with PDF severity $\left(r_{s}=0.43\right)$. However, this relationship is counterintuitive in that a negative association is expected if there were any relationship between an antiinflammatory cytokine and PDF severity. Our results are largely in accordance with the few existing comparative studies of Dreisbach et al. ${ }^{32}$ and Singh et al. ${ }^{33}$, who also did not find a significant relationship between PDF symptoms and peridialytic changes in pro-inflammatory IL-1 $\beta$ and TNF- $\alpha$ concentrations, respectively. A better understanding of the potential interplay between higher anti-inflammatory IL-10 levels before dialysis and the severity of PDF is an interesting objective for future research. Further, a potentially interesting avenue of investigation involves variations in genes related to inflammation. Indeed, cytokine genes are highly polymorphic and previous research in cancer patients showed that polymorphisms in cytokine genes influenced symptoms such as pain, depression and fatigue. ${ }^{38}$ Interestingly, preliminary evidence exists for a role of IL-10 gene polymorphisms in severe fatigue in lung cancer patients. ${ }^{38}$ Therefore, investigating the role of genetic variations in cytokine genes in the development of PDF or more chronic fatigue may advance our understanding and treatment of fatigue in HD patients. 
Taken together, our findings do not support the hypothesis that fatigue specifically occurring after HD treatment may be related to changes in pro- and anti-inflammatory cytokine levels as a result of the HD procedure. As such, there is currently insufficient evidence to consider anti-inflammatory therapy as a potential treatment for postdialysis fatigue. However, these results do not necessarily undermine the previously found relationship between inflammatory cytokine levels and the chronically fatigue symptoms commonly experienced by HD patients. Clearly, fatigue in HD patients is a complex phenomenon and fatigue as a response to treatment may have other determinants than the more chronically fatigue experience. For instance, recent research showed that several behavioural, social and psychological factors may be associated with fatigue experience in HD patients. ${ }^{39}$ Moreover, a recent study provided preliminary clinical evidence that HD-related fatigue may in part be explained as a conditioned response to the hospital environment. ${ }^{40}$ Therefore, it is appropriate to take into account both, biochemical and psychosocial variables, when trying to unravel the aetiology of fatigue in HD patients.

The present study has some limitations that need to be discussed. First, the inclusion of only two time-points for cytokine measurements might have masked transient, intradialytic or postdialytic rises in cytokine levels. Furthermore, the small panel of biomolecules analyzed in the study may omit information that could be gathered through the analysis of larger sets of analytes. Finally, the rather small sample size and the absence of a healthy control group to compare cytokine levels may further limit the generalizability of this study. 


\section{References}

1. Liyanage $T$, Ninomiya $T$, Jha $V$, et al. Worldwide access to treatment for end-stage kidney disease: a systematic review. Lancet (London, England). 2015;385(9981):1975-1982.

2. Heaf J. Current trends in European renal epidemiology. Clin Kidney J. 2017;10(2):149-153.

3. Kramer A, Pippias M, Noordzij M, et al. The European Renal Association - European Dialysis and Transplant Association (ERA-EDTA) Registry Annual Report 2015: a summary. Clin Kidney J. 2018;11(1):108-122.

4. United States Renal Data System. USRDS annual data report: Epidemiology of kidney disease in the United States. . 2018; https://www.usrds.org/2018/view/Default.aspx.

5. Bossola M, Tazza L. Postdialysis Fatigue: A Frequent and Debilitating Symptom. Seminars in dialysis. 2016;29(3):222-227.

6. Sklar AH, Riesenberg LA, Silber AK, Ahmed W, Ali A. Postdialysis fatigue. American journal of kidney diseases : the official journal of the National Kidney Foundation. 1996;28(5):732-736.

7. Sklar AH, Beezhold DH, Newman N, Hendrickson T, Dreisbach AW. Postdialysis fatigue: Lack of effect of a biocompatible membrane. American Journal of Kidney Diseases. 1998;31(6):1007-1010.

8. Sklar A, Newman N, Scott R, Semenyuk L, Schultz J, Fiacco V. Identification of factors responsible for postdialysis fatigue. American Journal of Kidney Diseases. 1999;34(3):464-470.

9. Dubin RF, Teerlink JR, Schiller NB, Alokozai D, Peralta CA, Johansen KL. Association of segmental wall motion abnormalities occurring during hemodialysis with post-dialysis fatigue. Nephrology, dialysis, transplantation : official publication of the European Dialysis and Transplant Association - European Renal Association. 2013;28(10):2580-2585.

10. Lindsay RM, Leitch R, Heidenheim AP, Kortas C. The London daily/nocturnal hemodialysis studystudy design, morbidity, and mortality results. American Journal of Kidney Diseases. 2003;42:5-12.

11. Bossola M, Marzetti E, Di Stasio E, et al. Prevalence and associated variables of post-dialysis fatigue: Results of a prospective multicentre study. Nephrology (Carlton, Vic.). 2018;23(6):552-558.

12. Bossola M, Di Stasio E, Antocicco M, Silvestri P, Tazza L. Variables associated with time of recovery after hemodialysis. Journal of nephrology. 2013;26(4):787-792.

13. Saligan LN, Kim HS. A systematic review of the association between immunogenomic markers and cancer-related fatigue. Brain Behav Immun. 2012;26(6):830-848.

14. Flachenecker P, Bihler I, Weber F, Gottschalk M, Toyka KV, Rieckmann P. Cytokine mRNA expression in patients with multiple sclerosis and fatigue. Multiple sclerosis (Houndmills, Basingstoke, England). 2004;10(2):165-169.

15. Heesen C, Nawrath L, Reich C, Bauer N, Schulz KH, Gold SM. Fatigue in multiple sclerosis: an example of cytokine mediated sickness behaviour? Journal of neurology, neurosurgery, and psychiatry. 2006;77(1):34-39.

16. Moreland LW, Genovese MC, Sato R, Singh A. Effect of etanercept on fatigue in patients with recent or established rheumatoid arthritis. Arthritis and rheumatism. 2006;55(2):287-293.

17. Fung A, Vizcaychipi $M$, Lloyd D, Wan $Y, M a D$. Central nervous system inflammation in disease related conditions: mechanistic prospects. Brain Res. 2012;1446:144-155.

18. Dantzer R. Cytokine-induced sickness behavior: mechanisms and implications. Annals of the New York Academy of Sciences. 2001;933:222-234.

19. Dantzer R, Kelley KW. Twenty years of research on cytokine-induced sickness behavior. Brain Behav Immun. 2007;21(2):153-160.

20. Karshikoff B, Sundelin T, Lasselin J. Role of Inflammation in Human Fatigue: Relevance of Multidimensional Assessments and Potential Neuronal Mechanisms. Frontiers in immunology. 2017;8:21. 
21. Norheim KB, Jonsson G, Omdal R. Biological mechanisms of chronic fatigue. Rheumatology (Oxford, England). 2011;50(6):1009-1018.

22. Dantzer R, O'Connor JC, Freund GG, Johnson RW, Kelley KW. From inflammation to sickness and depression: when the immune system subjugates the brain. Nature reviews. Neuroscience. 2008;9(1):46-56.

23. Yao Q, Lindholm B, Stenvinkel P. Inflammation as a cause of malnutrition, atherosclerotic cardiovascular disease, and poor outcome in hemodialysis patients. Hemodialysis international. International Symposium on Home Hemodialysis. 2004;8(2):118-129.

24. Kaysen GA. The microinflammatory state in uremia: causes and potential consequences. Journal of the American Society of Nephrology : JASN. 2001;12(7):1549-1557.

25. Bambauer R, Schmidt R, Falkenhagen D, Walther J, Jung WK. Sterile and endotoxin free dialysis fluid for hemodialysis. Biomaterials, artificial cells, and immobilization biotechnology : official journal of the International Society for Artificial Cells and Immobilization Biotechnology. 1991;19(1):71-83.

26. Wong J, Vilar E, Farrington K. Endotoxemia in end-stage kidney disease. Seminars in dialysis. 2015;28(1):59-67.

27. Vanholder R, Ringoir S, Dhondt A, Hakim R. Phagocytosis in uremic and hemodialysis patients: a prospective and cross sectional study. Kidney international. 1991;39(2):320-327.

28. Bossola M, Luciani G, Giungi S, Tazza L. Anorexia, fatigue, and plasma interleukin-6 levels in chronic hemodialysis patients. Renal failure. 2010;32(9):1049-1054.

29. Bossola M, Di Stasio E, Giungi S, Rosa F, Tazza L. Fatigue is associated with serum interleukin-6 levels and symptoms of depression in patients on chronic hemodialysis. J Pain Symptom Manage. 2015;49(3):578-585.

30. Bossola M, Luciani G, Tazza L. Fatigue and its correlates in chronic hemodialysis patients. Blood purification. 2009;28(3):245-252.

31. Brys ADH, Di Stasio E, Lenaert B, et al. Serum interleukin- 6 and endotoxin levels and their relationship with fatigue and depressive symptoms in patients on chronic haemodialysis. Cytokine. 2020;125:154823.

32. Dreisbach AW, Hendrickson T, Beezhold D, Riesenberg LA, Sklar AH. Elevated levels of tumor necrosis factor alpha in postdialysis fatigue. The International journal of artificial organs. 1998;21(2):83-86.

33. Singh NP, Bansal R, Thakur A, Kohli R, Bansal RC, Agarwal SK. Effect of membrane composition on cytokine production and clinical symptoms during hemodialysis: a crossover study. Renal failure. 2003;25(3):419-430.

34. Lindsay RM, Heidenheim PA, Nesrallah G, Garg AX, Suri R, Daily Hemodialysis Study Group London Health Sciences $C$. Minutes to recovery after a hemodialysis session: a simple health-related quality of life question that is reliable, valid, and sensitive to change. Clinical journal of the American Society of Nephrology : CJASN. 2006;1(5):952-959.

35. Dantzer R, Heijnen CJ, Kavelaars A, Laye S, Capuron L. The neuroimmune basis of fatigue. Trends Neurosci. 2014;37(1):39-46.

36. Wolley MJ, Hutchison CA. Large uremic toxins: an unsolved problem in end-stage kidney disease. Nephrology, dialysis, transplantation : official publication of the European Dialysis and Transplant Association - European Renal Association. 2018;33(suppl_3):iii6-iii11.

37. Aziz N, Detels R, Quint JJ, Li Q, Gjertson D, Butch AW. Stability of cytokines, chemokines and soluble activation markers in unprocessed blood stored under different conditions. Cytokine. 2016;84:17-24.

38. Reyes-Gibby CC, Wang J, Spitz M, Wu X, Yennurajalingam S, Shete S. Genetic variations in interleukin8 and interleukin-10 are associated with pain, depressed mood, and fatigue in lung cancer patients. $J$ Pain Symptom Manage. 2013;46(2):161-172. 
39. Brys A, Stifft F, van Heugten C, Bossola M, Gambaro G, Lenaert B. mHealth based Experience Sampling Method to identify fatigue in the context of daily life in hemodialysis patients. Clin Kidney J. 2020;in press.

40. Brys $A D H$, Lenaert B, Van Heugten CM, Gambaro G, Bossola M. Exploring the Diurnal Course of Fatigue in Patients on Hemodialysis Treatment and Its Relation With Depressive Symptoms and Classical Conditioning. J Pain Symptom Manage. 2019;57(5):890-898.e894. 

Part II

The relationship of psychological and contextual variables with fatigue in chronic haemodialysis patients 



\section{Chapter 4}

Exploring the diurnal course of fatigue in patients on haemodialysis treatment and its relation with depressive symptoms and classical conditioning

Brys ADH, Lenaert B, Van Heugten CM, Gambaro G, Bossola M Published in J Pain Symptom Manage. 2019;57(5):890-898.e4 


\begin{abstract}

\section{Background}

Fatigue is one of the most prevalent symptoms among haemodialysis (HD) patients. In order to design effective treatments it is crucial to understand the diurnal pattern of fatigue in this population.
\end{abstract}

\title{
Objectives
}

To assess diurnal changes in fatigue in patients undergoing haemodialysis and their relation with depressive symptoms. To explore whether fatigue may become a classically conditioned response to the hospital environment.

\section{Methods}

A prospective, observational study was conducted in fifty-one HD patients. Subjects repeatedly rated their current fatigue on three different days during one week of haemodialysis treatment to capture changes in momentary fatigue. First, on a HD treatment day, fatigue was measured one hour before and immediately prior to dialysis, as well as immediately after dialysis and again at 22:00 p.m. Second, on the postdialysis day and on the seventh weekday (when patients had not received treatment on the previous day), fatigue was measured at the same moments in time as the two measurements before dialysis on the treatment day. Beck Depression Inventory-II and Fatigue Severity Scale were administered to evaluate depressive mood and fatigue severity in daily life.

\section{Results}

Fatigue increased as a result of haemodialysis treatment over the entire sample. However, diurnal fatigue patterns differed significantly between individuals high and low in depressive symptoms, with the former being fatigued more constantly throughout the day, and the latter experiencing increases in fatigue due to treatment. Pre-treatment fatigue experienced in the hospital environment followed a pattern consistent with the development of a classically conditioned response.

\section{Conclusions}

Diurnal fatigue patterns during haemodialysis treatment are associated with depressive symptoms, and classical conditioning may play a role in the experience of pre-treatment fatigue. 


\section{Introduction}

The prevalence of renal replacement therapy (RRT) in Europe and the USA is estimated to be 924 and 2274 per million population, respectively. ${ }^{1,2}$ Haemodialysis (HD) is by far the most prevalent RRT in the world. ${ }^{3}$ The symptom burden of HD patients is comparable to that of terminal cancer patients. ${ }^{4,5}$ Fatigue is one of the most common symptoms among HD patients, greatly affecting health related quality of life. ${ }^{6,7}$ Since $40-80 \%$ of HD patients suffer from fatigue and HD treatment is usually received for many years, the need for effective fatigue interventions is beyond dispute. $^{7}$ It is therefore crucial to understand the development and course of fatigue in HD patients.

However, as an inherently subjective experience, fatigue is difficult to capture and define. A commonly used definition refers to fatigue as "an overwhelming, debilitating and sustained sense of exhaustion that is likely to decrease one's ability to carry out daily activities, including the ability to work effectively and to function at one's usual level in family or social roles" ${ }^{8,9}$ Fatigue in patients on HD treatment in particular is a complex and multidimensional phenomenon. For instance, different fatigue qualities can be distinguished, such as physical (weakness, tiredness, lack of energy) and mental (emotional, cognitive) fatigue, with the former being reported more frequently in this population. ${ }^{10,11}$ Furthermore, fatigue in individuals undergoing HD treatment may be determined by multiple factors. Indeed, acute fatigue as a response to treatment may have different determinants than fatigue that is experienced more chronically by HD patients. ${ }^{12}$ The difficulty to identify and study fatigue in HD patients is enhanced by the lack of a valid and reliable fatigue scale specifically developed for the end-stage kidney disease (ESKD) population. Recently, a systematic review on fatigue instruments employed in haemodialysis, found that fatigue was measured in 43 different ways across 123 studies. ${ }^{13}$ Moreover, the most commonly used fatigue measures (e.g. Fatigue Severity Scale (FSS) or SF-36 vitality subscale) only evaluate the overall fatigue experience retrospectively, for instance over the preceding week or month. As such, the implementation of novel measuring methods, like Ecological Momentary Assessment (EMA) procedures, taking into account the diurnal variability of fatigue by repeatedly collecting real-time measurements of fatigue over the course of several days, is warranted. To date, only a limited number of studies reported the use of EMA to evaluate the variability of fatigue in HD patients. These studies showed 
indeed differences in fatigue between as well as within individuals, for instance on treatment versus non-treatment days. However, none of these reports investigated the relationship between diurnal fatigue patterns and variables that may explain these differences. ${ }^{14-17}$

Additionally, research findings are inconsistent regarding the relation between fatigue in HD patients and physiological, psychological, socio-demographic or dialysis-related variables. No causal association has been demonstrated yet. ${ }^{18,19}$ As such, a better understanding of the factors contributing to fatigue in HD patients is crucial for the development of effective treatments. Interestingly, a biopsychosocial model of fatigue in HD patients has recently been proposed. In this model, fatigue is initially triggered by biochemical imbalances or stress and worrying, and then maintained by a vicious cycle of negative beliefs, depression and anxiety, and maladaptive behavioural patterns such as avoidance of activity and sleep problems. ${ }^{19}$ Previous reports already demonstrated a significant association between general, retrospectively reported fatigue and depression in HD patients. ${ }^{20,21}$ However, the relationship between depressive symptoms and momentary changes in fatigue related to the dialysis treatment has not been investigated before. In order to better understand fatigue in response to treatment in patients who undergo haemodialysis thrice weekly, it is important to assess whether individual differences in depressive symptoms contribute to momentary fatigue related to haemodialysis. Therefore, the first aim of the present study was to assess the diurnal change in fatigue in response to haemodialysis and its relation with depressive symptoms.

Within a biopsychosocial approach to fatigue in HD patients and as a second aim of this study, we put forward classical conditioning as another mechanism that may contribute to the maintenance of HD-related fatigue. Classical conditioning can be defined as a change in behaviour to a stimulus or event due to its pairing with another stimulus or event. ${ }^{22}$ Indeed, clinical evidence for fatigue as a classically conditioned response has already been shown in breast cancer patients receiving repeated infusions of chemotherapy. Bovbjerg et al. demonstrated that the repeated pairing of the hospital environment with chemotherapy and its undesirable side effects such as fatigue and nausea may result in the experience of these symptoms prior to chemotherapy. This can be explained as a classically conditioned response, where the hospital environment became a conditioned stimulus capable of eliciting these 
symptoms due to its previous pairing with chemotherapy. As such, classical conditioning may loosen the link between treatment and the experience of fatigue, thereby potentially contributing to the fatigue becoming a chronic symptom, above and beyond pathophysiological factors. ${ }^{23,24}$ Therefore, our second aim was to explore whether HD-related fatigue may also in part be explained as a conditioned response to the hospital environment that has become associated with haemodialysis treatment. To this aim, we recorded fatigue ratings immediately before haemodialysis on treatment days in the hospital environment and compared these with fatigue measured at the same hour of the day on non-treatment days. We predicted that pretreatment fatigue would be higher on a treatment day in the hospital environment than fatigue on non-treatment days measured at the same time of day outside of the hospital environment.

\section{Subjects and methods}

\section{Study population}

All prevalent chronic patients of the HD unit of the Catholic University of Rome (Italy) between June and August 2018 were considered eligible for inclusion in this observational study. Exclusion criteria were as follows: $<18$ years of age, inability to answer the questionnaires because of hearing or reading problems, dementia, actual instability of clinical condition requiring hospitalization or active cancer. The local ethics committee approved the study protocol (13615/18-22147/18). All participants gave their informed consent.

\section{Data collection}

A fatigue diary was developed to prospectively and repeatedly measure momentary fatigue on eight different moments during one week of regular treatment. First, to assess diurnal changes in fatigue on a HD treatment day, fatigue was measured one hour before dialysis, as well as immediately before dialysis started at the dialysis unit. Fatigue was measured again after termination of the dialysis session at the dialysis unit, and finally at 22:00 p.m. Second, to assess classical conditioning effects, fatigue was measured again on a postdialysis day at exactly the same moment as the fatigue measurement immediately before dialysis in the hospital environment. Fatigue rating 
was also collected one hour earlier as control measurement. These two measurements were repeated on the seventh weekday (when patients had not received treatment on the previous day) (Figure 4.1). Fatigue was rated on an 11-point Likert scale from zero (no fatigue at all) to ten (extremely fatigued).

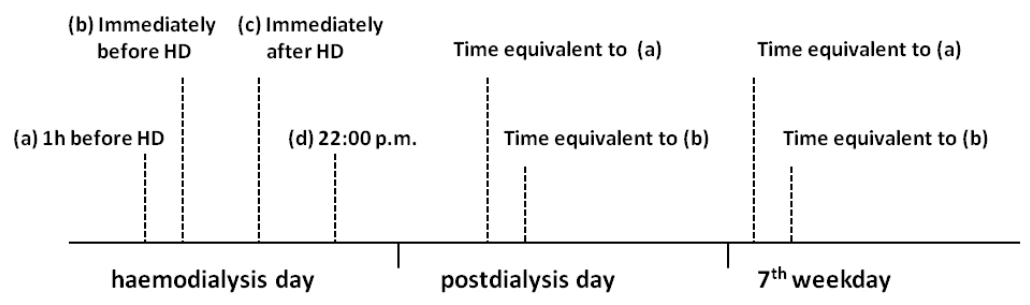

Figure 4.1 Fatigue diary: time point assessment.

The Italian versions of the Beck Depression Inventory-II (BDI-II) and Fatigue Severity Scale (FSS) were administered to evaluate depressive mood and the impact of general fatigue on the activities of daily living during the past seven days. The BDI-II is a 21-item, patient-rated scale that has been validated in the haemodialysis population. ${ }^{25}$ Scores can range from $0-63$, with higher scores indicating more severe depressive symptoms. The FSS is a 9-item questionnaire with a seven-point Likert scale. The total score is calculated by averaging the scores of the individual item responses. The scores can range from 1 (no fatigue) to 7 (maximum fatigue). ${ }^{26}$ The FSS is one of the most frequently used measuring instruments to evaluate fatigue in renal patients. ${ }^{27}$ Moreover, we preferred administering the FSS over other measuring instruments, like the Edmonton Symptom Assessment System renal survey (ESASrenal) or Integrated Palliative care Outcome Scale renal survey (IPOS-renal), as they evaluate the overall symptom burden of renal patients and dedicate only one item to fatigue in particular.

The following demographical, clinical and laboratory information was obtained from medical records: age, gender, dialysis vintage, primary cause of ESKD, duration of dialysis, type and number of comorbidities assessed by the Charlson Comorbidity index, ${ }^{28}$ self reported functional status via de Activity of daily living (ADL) scale ${ }^{29}$ and time of recovery after haemodialysis (TIRD) in minutes, ${ }^{30}$ haemoglobin, sodium, calcium, phosphorus, PTH, creatinine and dialysis dose as evaluated by $K t / \mathrm{V}$. 


\section{Procedure}

After giving their informed consent, each participant received their personal diary along with verbal and written instructions and started data collection the following day. The BDI-II and FSS questionnaires were completed on the treatment day during dialysis. Demographical and clinical information was obtained at the moment of inclusion. Results of monthly routine biochemical and haematological investigation were obtained closest to the day on which the questionnaires were completed.

\section{Haemodialysis treatment}

Patients received bicarbonate haemodialysis treatment between 3.5-4 hours, twice or thrice weekly. The blood flow ranged from $250-300 \mathrm{ml} / \mathrm{min}$ with a dialysis rate flow of $500 \mathrm{ml} / \mathrm{min}$. High permeability membranes were used.

\section{Statistical analysis}

Statistical analysis was performed by using the Statistical Package for Social Science (IBM SPSS Statistics 25). Data are presented as mean and standard deviation (SD) and statistical significance was assessed at $p<0.05$. To investigate diurnal changes in fatigue in HD patients and their relation with depressive symptoms, we performed a mixed ANOVA with reported fatigue as dependent variable, measurement moment on the day of treatment as within-subjects factor and a low or high presence of depressive symptoms (BDI-II score $\leq 13$ versus BDI-II $>13$, corresponding to the cut-off score to classify for depressive mood according to the guidelines to interpret the $\left.B D I-I^{31}\right)$, as between-subjects factor. Whenever Mauchly's test indicated that the assumption of sphericity was violated, F-ratio, degrees of freedom, and $p$-values are reported with the applied Huynh-Feldt corrections (all epsilons $>0.75$ ). To evaluate the role of classical conditioning, we performed a mixed ANOVA with fatigue immediately before dialysis (or corresponding moment on non-treatment days) as dependent variable and day (treatment day, postdialysis day, $7^{\text {th }}$ weekday) as within-subjects factor. 


\section{Results}

\section{Patient characteristics}

Sixty-three patients were approached of whom fifty-seven individuals provided informed consent ( $90.5 \%$ consent rate). Subsequently, three withdrew due to loss of interest, one patient withdrew due to hospitalization and two were excluded from the analyses due to poor adherence to the study protocol (instead of recording momentary fatigue ratings throughout the week, these two subjects filled in the entire diary immediately before handing it in). The remaining fifty-one individuals with a mean age of 58.70 years $(S D=18.70)$ are included in this report. Baseline demographical, clinical and laboratory characteristics as well as BDI-II and FSS scores are reported in Table 4.1. The fatigue severity score, reported by this HD population, is almost twice as high as compared to previous estimates of fatigue in healthy adults with a mean (SD) FSS score of $2.3(0.7) .{ }^{26}$ Correlations between the above mentioned demographical and dialysis-related variables and the mean of all eight momentary fatigue ratings (representing the mean fatigue experienced during the week of the study) are reported in the Supplemental Table S4.1. Mean fatigue only correlated significantly with TIRD, indicating that patients who report having to recover for a longer time after dialysis, are on average more fatigued.

\section{Fatigue course in HD patients}

Mean fatigue scores (SD) on the HD treatment day were respectively 3.92 (2.59), 95\% $\mathrm{Cl}[3.19,4.65]$ one hour before dialysis, 4.14 (2.84), 95\% Cl [3.34, 4.94] immediately before dialysis, 5.08 (2.53), 95\% Cl [4.37, 5.79] immediately after dialysis and 5.45 (2.75), 95\% Cl $[4.68,6.22]$ at 22:00 p.m. Results of the mixed ANOVA showed a significant main effect of the measurement moment on fatigue rating, $F(2.67$, 131.91)=9.44, $p<0.001$, partial $\eta^{2}=0.159$. Planned comparisons of the four measurement moments revealed a significant fatigue increase from immediately before to immediately after haemodialysis treatment, $F(1,50)=6.75, p=0.012$, partial $\eta^{2}=0.119$. There were no significant differences between the measurement one hour before dialysis and the second measurement immediately before treatment, $F(1,50)=0.97, p=0.330$, partial $\eta^{2}=0.019$, nor between the postdialysis measurement moment and the measurement at 22:00 p.m., $F(1,50)=1.07, p=0.305$, partial $\eta^{2}=0.021$ (Figure 4.2). Furthermore, fatigue severity in daily life, assessed with the FSS, did not 
correlate with the momentary fatigue rating after HD treatment $(r=0.12, p=0.371)$. Nevertheless, the FSS-score was significantly, although moderately, associated with the average of all other fatigue ratings individuals reported. $(r=0.34, p=0.017)$.

Table 4.1 Baseline demographical, clinical and laboratory characteristics $(N=51)$.

\begin{tabular}{lc}
\hline Characteristics & Mean \pm SD [range] / (n) frequency \\
\hline Age(years) & $58.7(18.7)[20-91]$ \\
Sex (men) & $28(55 \%)$ \\
Dialysis vintage, months & $70(68.7)[7-386]$ \\
Hours of dialysis /week & $11.4(1.2)$ \\
Primary cause of ESRD & \\
hypertension & $12(24 \%)$ \\
glomerulonephritis & $15(29 \%)$ \\
diabetes & $8(16 \%)$ \\
interstitial nephritis & $2(3 \%)$ \\
polycystic kidney disease & $5(10 \%)$ \\
others/unknown & $9(18 \%)$ \\
Charlson Comorbidity Index (CCI) & $1.5(1.4)[0-6]$ \\
Activity of Daily Living (ADL) & $5.8(0.7)[2-6]$ \\
Morning/afternoon treatment & $23 / 28(45 \%)$ \\
Beck Depression Inventory-II (BDI) & $15.4(8.4)[2-33]$ \\
Fatigue Severity Scale (FSS) & $4.3(1.5)[1.3-6.6]$ \\
TIRD (minutes) (median) & $180(674)[10-4320]$ \\
UFR (/ml/kg/h) & $8.4(3.2)$ \\
Kt/V & $1.52(0.4)$ \\
Haemoglobin (g/dl) & $10.6(1.0)$ \\
Serum Creatinine (mg/dl) & $10.5(2.7)$ \\
Calcium (mg/dl) & $9.2(0.8)$ \\
Serum Sodium (mmol/l) & $138.9(2.9)$ \\
Dialysate Sodium (mmol/l) & $138.7(1.9)$ \\
Parathyroid hormone (pg/ml) & $570(455)$ \\
Phosphorus (mg/dl) & $5.3(1.4)$ \\
\hline
\end{tabular}

TIRD: Time of Recovery after Dialysis, UFR: Ultrafiltration rate, $K t / V$ : urea clearance 

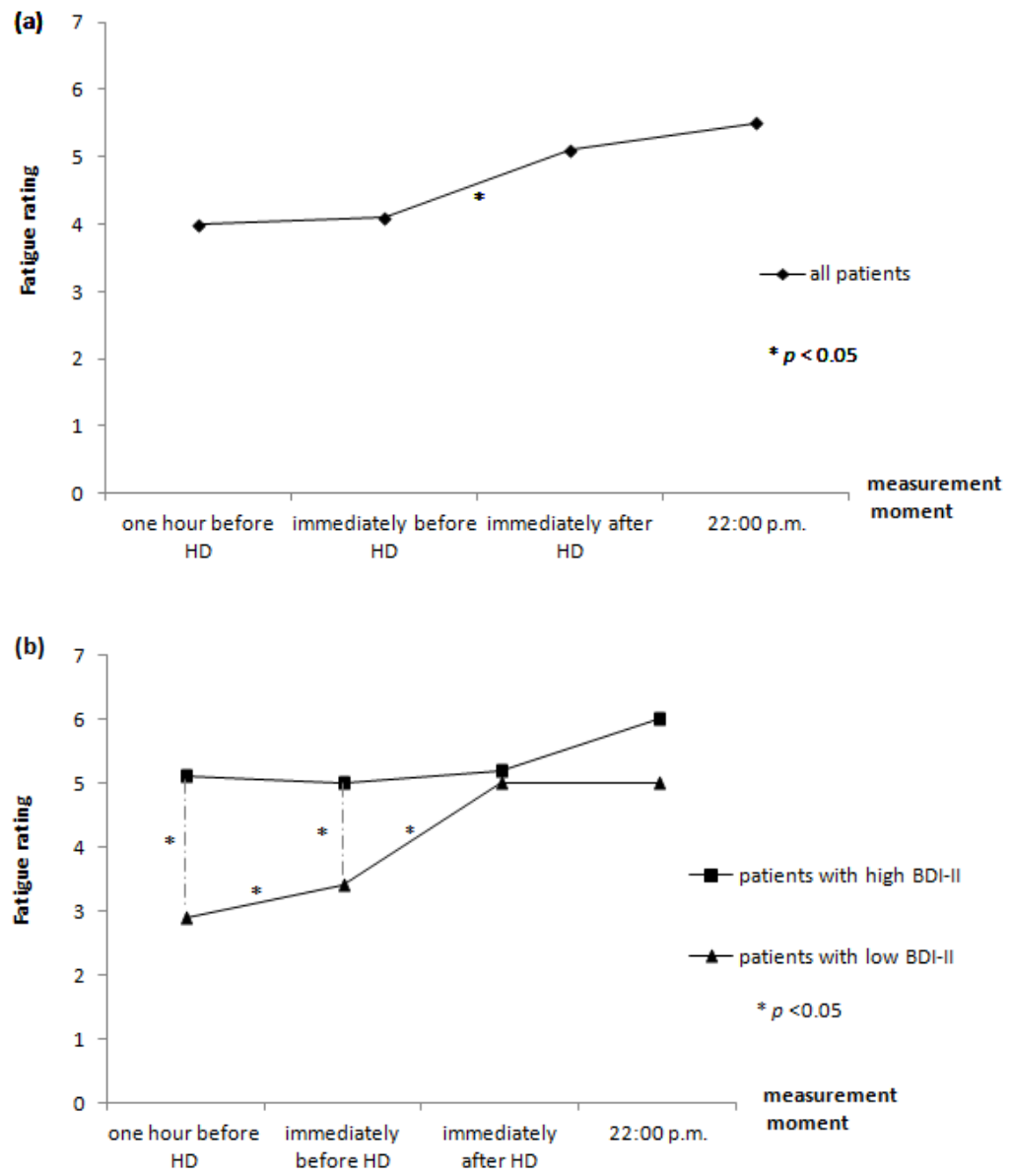

Figure 4.2 Course of fatigue on a HD treatment day (a) and its relation to depressive symptoms (b). a) Fatigue levels in HD patients vary according to the measurement moment on the HD day. A marked increase in fatigue was found immediately after the HD session. b) Individuals high in depressive symptoms (BDI-II score of $>13$ ), reported continuously high levels of fatigue. Lowdepressed individuals (BDI-II score $\leq 13$ ) were significantly less tired than high-depressed individuals before treatment, but showed a marked increase in fatigue after HD treatment. ${ }^{*} p<0.05$. 


\section{Fatigue course in HD patients and its relation to depression}

For this analysis, depressive status (BDI-II) was added as between-subjects factor, based on the cut-off score described earlier. Results are based on 50 subjects, because one individual declined to fill in the questionnaire, resulting in a group high in depressive symptoms $(n=25)$ and a group low in depressive symptoms $(n=25)$. The low-depressed patient group had a mean (SD) BDI-II score of 8.76 (2.99), whereas the high-depressed patient group had a mean (SD) BDI score of 22 (6.68). Mean diurnal fatigue scores (SD) on the consecutive measurement moments are reported in Table 2. We found no main effect of depression, $F(1,48)=3.95, p=0.053$, partial $\eta^{2}=0.076$. However, there was a significant measurement moment $\times$ depression interaction, $F(2.71,130.18)=3.36, p=0.025$, partial $\eta^{2}=0.065$, showing that fatigue throughout the day of HD treatment differed depending on one's depressive symptoms. Comparison between low-depressed and high-depressed patients showed a significantly different fatigue rating one hour before dialysis and immediately before HD started, $F(1$, $48)=10.70, p=0.002$, partial $\eta^{2}=0.182$ and $F(1,48)=4.12, p=0.048$, partial $\eta^{2}=0.079$, respectively. It is noteworthy that this pattern of results was also found on nontreatment days. Here, fatigue ratings were collected at the same hours of the day as the fatigue ratings one hour before and immediately preceding haemodialysis on the treatment day in order to answer our research question about classical conditioning. Interestingly, individuals high in depressive symptoms reported higher fatigue than low-depressed individuals on all of these time points as well. (Results in Supplemental Table S4.2).

Table 4.2 Fatigue difference between high- and low-depressed individuals according to the Beck Depression Inventory-II (BDI) on a haemodialysis (HD) treatment day ( $\mathrm{N}=50)$.

\begin{tabular}{lccc}
\hline Haemodialysis day & Low BDI Mean (SD) [95\% CI] & High BDI Mean (SD) [95\% CI] & $p$ \\
\hline One hour before HD & $2.92(2.50)[1.89,3.95]$ & $5.08(2.16)[4.19,5.97]$ & 0.002 \\
Immediately before HD & $3.44(2.75)[2.30,4.58]$ & $5.00(2.68)[3.90,6.11]$ & 0.048 \\
Immediately after HD & $5.04(1.93)[4.25,5.84]$ & $5.16(3.09)[3.88,6.44]$ & 0.870 \\
22:00 p.m. & $5.00(2.86)[3.82,6.18]$ & $6.00(2.60)[4.93,7.07]$ & 0.200 \\
\hline
\end{tabular}

Further, in the low-depressed group, there was a significant increase in fatigue from one hour before HD to immediately before $H D, F(1,24)=8.90, p=0.006$, partial $\eta^{2}=0.270$. Likewise, there was a significant increase from pre-treatment to posttreatment fatigue, $F(1,24)=12.39, p=0.002$, partial $\eta^{2}=0.340$. There was no significant fatigue rating difference in this group between the measurement just after HD and 
the one at 22:00 p.m., $F(1,24)=0.01, p=0.930$, partial $\eta^{2}=0.000$. In contrast, in the high-depressed group, there were no significant differences in fatigue levels between any of the consecutive measurement moments, $F(1,24)=0.04, p=0.846$, partial $\eta^{2}=0.002$ (measurement moment 1 versus 2), $F(1,24)=0.09, p=0.770$, partial $\eta^{2}=0.004$ (moment 2 versus 3 ) and $F(1,24)=2.15, p=0.156$, partial $\eta^{2}=0.082$ (moment 3 versus 4 ) (Figure 4.2).

\section{Fatigue in HD patients and classical conditioning}

This analysis was based on 48 subjects, because three individuals did not report their fatigue rating on either the postdialysis day or the $7^{\text {th }}$ weekday. Mean fatigue scores (SD) were $4.10(2.79), 95 \% \mathrm{Cl}[3.29,4.16]$ immediately before HD started on the treatment day, $3.00(2.70), 95 \% \mathrm{Cl}[2.22,3.78]$ on the postdialysis day, and 3.38 (2.76), $95 \% \mathrm{Cl}[2.57,4.18]$ on the $7^{\text {th }}$ weekday, measured at the same time of day respectively. Mixed ANOVA revealed a main effect of day, $F(2,94)=5.58, p=0.005$, partial $\eta^{2}=0.106$. Planned comparisons revealed that fatigue immediately before haemodialysis in the hospital environment was significantly higher than fatigue at the same time of day on the postdialysis day, $F(1,47)=9.34, p=0.004$, partial $\eta^{2}=0.166$, and the $7^{\text {th }}$ weekday $F(1,47)=4.48, p=0.040$, partial $\eta^{2}=0.087$. No significant difference in fatigue rating was found between the postdialysis day and the $7^{\text {th }}$ weekday, $F(1,47)=0.44, p=0.512$, partial $\eta^{2}=0.009$. In contrast, a mixed ANOVA with the control fatigue measurement taken in a different environment than the hospital one hour earlier revealed no differences in fatigue between the treatment day, postdialysis day or $7^{\text {th }}$ weekday, $F(2,96)=1.94, p=0.149$, partial $\eta^{2}=0.039$ (Figure 4.3). 


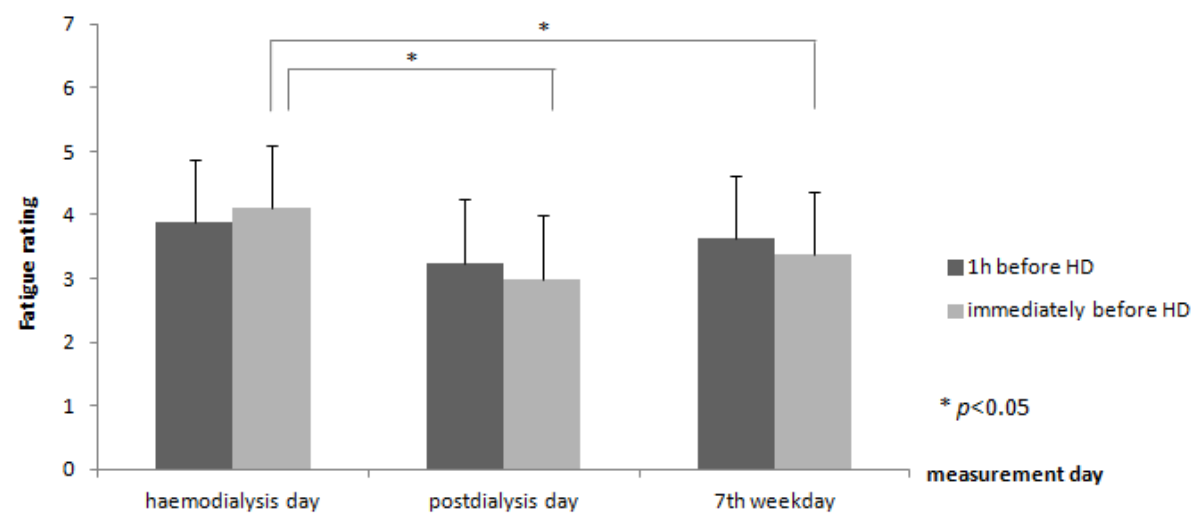

Figure 4.3 Exploration of classical conditioning in HD related fatigue $(N=48)$. Momentary fatigue measurements one hour before and immediately before HD on a treatment day, postdialysis day and $7^{\text {th }}$ weekday. Fatigue levels immediately before HD in the hospital environment were significantly higher than fatigue at the same time of the day on a postdialysis day and the $7^{\text {th }}$ weekday. On the contrary, control fatigue measurement in a different environment than the hospital one hour earlier showed no differences in fatigue between the different days. To control for carryover effects, the order of the day in which individuals completed the fatigue diary varied: one third of participants started on a dialysis treatment day, another third started on a postdialysis day and the remaining third started on the seventh weekday. They all completed the diary within one week of regular treatment. ${ }^{*} p<0.05$.

\section{Discussion}

The current study was conducted to assess diurnal changes in fatigue in response to haemodialysis treatment and their relation with depressive symptoms. Furthermore, we explored whether HD-related fatigue may in part be explained as a conditioned response to the hospital environment. We found that fatigue increases significantly during HD treatment, but only in low-depressed individuals according to the BDI-II. In contrast, individuals high in depressive symptoms already reported high levels of fatigue before treatment, and these were not significantly increased afterwards. We also showed that patients reported higher fatigue levels in the hospital environment prior to treatment relative to other environments that are not associated with haemodialysis, which is in line with the development of a classically conditioned fatigue response.

First, our results show that fatigue increased significantly during a treatment day and especially during the dialysis session. These findings are in line with a previous study 
of Abdel-Kader et al. ${ }^{14}$ who also demonstrated diurnal increases in fatigue in HD patients. However, their study did not specifically investigate fatigue levels immediately before and after HD, nor did they investigate the relationship of fatigue with depressive symptoms. In addition, we found that postdialysis fatigue did not correlate with fatigue severity in general as measured with the FSS. However, FSS scores were moderately associated with the average of all other (i.e. not postdialysis) fatigue ratings in our study. This supports the finding of different fatigue patterns (i.e. more general fatigue versus fatigue as a response to treatment) and may suggest that these should, at least partially, be distinguished in HD patients. ${ }^{12,17}$ It also emphasises the need for a reliable fatigue measuring instrument adapted to the HD population, taking into account the general and treatment-related fatigue qualities in these individuals. $^{19}$ Moreover, conventional fatigue scales, evaluating fatigue retrospectively, are liable to recall bias and do not accurately reflect momentary fatigue. ${ }^{32}$ Interestingly, a new outcome measure for fatigue in haemodialysis is being developed by the International Standardized Outcomes in Nephrology (SONG) initiative, which identified fatigue as a core outcome domain in HD, in order to facilitate consistent measurement of fatigue in HD patients and facilitate comparison of the effects of interventions for fatigue across trials. ${ }^{33}$

Second, previous studies have revealed an association between general fatigue and depressive symptoms in HD patients. ${ }^{11,20,21,34,35}$ Here, we found that individuals high and low in depressive symptoms, reported similar levels of fatigue after HD treatment, but high-depressed individuals showed significantly higher fatigue before treatment relative to their low-depressed counterparts. Moreover, individuals high in depressive symptoms also experienced more fatigue than low-depressed individuals on non-treatment days. Thus, individuals high in depressive symptoms reported continuously high levels of fatigue, which appeared to be no longer directly affected by the HD treatment. In contrast, low-depressed individuals were less tired than highdepressed individuals, but showed a marked increase in fatigue after HD treatment. This may be seen as an adaptive response by inducing rest in order to conserve energy and thus allow recovery to take place. ${ }^{36}$ These findings are in line with the recently proposed biopsychosocial model of fatigue in ESKD. ${ }^{19}$ That is, fatigue symptoms may initially be triggered by chemical imbalances or may be caused by HD treatment itself, but may eventually be perpetuated by other factors such as depressive mood. To the extent that depressive mood contributes to the maintenance of fatigue in HD 
patients, interventions aimed at decreasing depressive symptoms may also be effective in alleviating fatigue. An interesting hypothesis is that successful treatment of depression may lead to a pattern similar to what was found in low-depressed individuals in this study, where fatigue increases after HD treatment, but is less present pre-treatment or on non-treatment days.

Third, in order to assess fatigue as a classically conditioned response, we used a different approach to that used by Bovbjerg and colleagues. ${ }^{23}$ In their study, patients were followed from the start of treatment onwards and evidence for a conditioned fatigue response was inferred from an increase in pre-chemotherapy fatigue over the course of treatment. In our study sample, however, prevalent HD patients were recruited, with a median dialysis vintage of 48 months, so an increase in pretreatment fatigue over the course of our study was not to be expected. Therefore, we chose an alternative approach that consisted of comparing fatigue on a treatment day to fatigue on non-treatment days. To the extent that the hospital environment - through repeated pairings with haemodialysis and ensuing fatigue increase - had effectively become a conditioned stimulus for fatigue, one would predict higher levels of fatigue in this environment relative to other environments that have not been paired with treatment. Results are in line with this prediction, thereby providing preliminary clinical evidence for fatigue as a conditioned response in individuals undergoing HD treatment.

However, some caution is warranted when interpreting these results as they allow alternative explanations. For instance, coming to the hospital (e.g. by means of transport) may require effort that also leads to increases in fatigue that do not necessarily require an explanation in terms of classical conditioning (but neither would it oppose such explanation, in that transport prior to treatment may also become a conditioned stimulus). However, given that most individuals in our sample engaged in professional or social activities on non-treatment days, it is unlikely that results should be explained solely in terms of day-dependent levels of activity or effort. Similar to Bovbjerg's study, future studies could include incident dialysis patients, which would allow assessing classical conditioning in terms of an increase in pre-treatment fatigue over the course of treatment. Future studies could also investigate the extent to which other conditioned responses than fatigue may develop. For instance, through repeated pairings with HD treatment, the hospital 
environment may not only trigger fatigue as a conditioned response, but also (and perhaps even more likely) as learned responses such as the expectancy of fatigue, and fear of fatigue to the extent that fatigue is experienced as an aversive symptom. Based on the literature on placebo and nocebo responses, it follows that the expectancy of fatigue may contribute to the fatigue experience. ${ }^{37}$ Moreover, anxious anticipation of fatigue may be associated with stress responses that may also increase fatigue.

This study adds to the existing literature by capturing the diurnal variability of fatigue by means of repeated measurements in the context of daily life. However, some limitations should be highlighted. First, because patients completed the fatigue diary without supervision, we could only assess compliance in terms of responses completed, but not in terms of the requested time of day of those responses. Future studies using EMA procedures incorporated in a web-application that record time stamps for each response can control for this. ${ }^{14}$ Second, we did not differentiate between different fatigue qualities such as mental or physical fatigue. Third, this was a single centre study with a rather small study sample that was followed for a period of one week. The generalizability of this study needs to be confirmed in future research. Fourth, we cannot draw any conclusions about a causal relationship between fatigue and depressive mood because our study design does not allow causal inferences.

Future studies using EMA procedures might help to better understand the course and development of fatigue in HD patients, the relation to haemodialysis treatment and the association with other factors. Additionally, this opens a window for psychological intervention studies to assess the effect on fatigue in HD patients by improving their mood, or vice versa. 


\section{Supplemental material}

Table S4.1 Correlation analyses between the mean of all eight momentary fatigue ratings and demographical, clinical and laboratory variables $(\mathrm{N}=48)$.

\begin{tabular}{lcc}
\hline Variable & Mean of all 8 momentary fatigue ratings \\
& $\boldsymbol{r}$ & $\boldsymbol{p}$ \\
\hline Age & 0.20 & 0.173 \\
Activity of Daily Living (ADL) & -0.04 & 0.809 \\
Recovery time after dialysis (minutes) (TIRD) & $0.39^{* *}$ & 0.007 \\
Dialysis vintage & 0.25 & 0.088 \\
Hours HD/week & 0.06 & 0.686 \\
Kt/V: urea clearance & 0.04 & 0.825 \\
Haemoglobin g/dl & 0.22 & 0.136 \\
Calcium mg/dl & 0.14 & 0.356 \\
Creatinine mg/dl & -0.20 & 0.187 \\
Sodium serum mmol/l & -0.05 & 0.768 \\
Phosphorus mg/dl & -0.17 & 0.263 \\
Parathyroid hormone $\mathrm{pg} / \mathrm{ml}$ & -0.07 & 0.634 \\
\hline
\end{tabular}

Mean (SD) of all eight momentary fatigue ratings: $3.96(2.14) ;{ }^{* *} p<0.01$. A one way ANOVA with the following variables was computed: Sex: $F(1,47)=2.543, p=0.124$, partial $\eta^{2}=0.051$; Morning-afternoon dialysis session: $F(1,47)=1.334, p=0.254$, partial $\eta^{2}=0.028$.

Table S4.2 Fatigue difference between high- and low-depressed individuals on non-treatment days $(\mathrm{N}=48)$.

\begin{tabular}{lcc}
\hline Postdialysis day & Low BDI-II Mean (SE) & High BDI-II Mean (SE) \\
\hline One hour before HD & $2.48(0.45)$ & $4.08(0.63)$ \\
Immediately before HD & $2.13(0.42)$ & $3.79(0.63)$ \\
\hline
\end{tabular}

BDI-II: Beck Depression Inventory II score (low $\leq 13$, high $>13$ ). On the postdialysis day at exactly the same time point as one hour before HD on the treatment day, patients with depressive symptoms experienced a higher fatigue level $(M=4.08, S E=0.63)$ than patients without depressive symptoms according to the BDI-II $(M=2.48, S E=0.45)$. This difference was significant, $t(47)=-2.09, p=0.044$. The same was found one hour later corresponding to the time point immediately before dialysis on the treatment day, with $t(46)=-2.20$, $p=0.034$. (Supplemental Figure S4.1: ${ }^{*} p<0.05$ )

\begin{tabular}{lcc}
\hline Seventh weekday & Low BDI-II Mean (SE) & High BDI-II Mean (SE) \\
\hline One hour before HD & $2.75(0.50)$ & $4.50(0.53)$ \\
Immediately before HD & $2.57(0.50)$ & $4.24(0.60)$ \\
\hline
\end{tabular}

On the seventh weekday, at the same time points as one hour before and immediately before HD on the treatment day, patients with depressive symptoms experience higher fatigue levels $(M=4.50, S E=0.53$ and $M=4.24, S E=0.60$, respectively) than patients without depressive symptoms $(M=2.75, S E=0.50$ and $M=2.57$, $S E=0.50)$. These differences were also significant with $t(46)=0.239, p=0.021$ and $t(46)=-2.12, p=0.040$. (Supplemental Figure S4.1: ${ }^{*} p<0.05$ ). Noteworthy, an additional correlation was found between BDI-II score and age in our patient group $(r=.369, p=0.008)$. The older patients were, the more they experienced depressive symptoms. 
Chapter 4

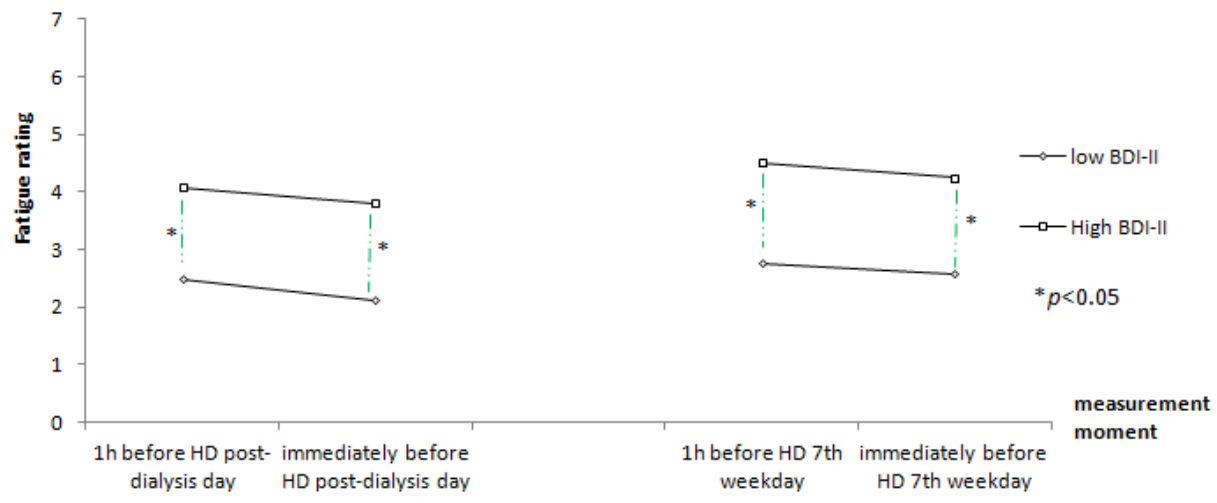

Figure S4.1 Fatigue levels in low- and high-depressed individuals on the postdialysis day and $7^{\text {th }}$ week day. 


\section{References}

1. Pippias M, Kramer A, Noordzij M, et al. The European Renal Association - European Dialysis and Transplant Association Registry Annual Report 2014: a summary. Clin Kidney J. 2017;10(2):154-169.

2. United States Renal Data System. USRDS annual data report: Epidemiology of kidney disease in the United States. . 2018; https://www.usrds.org/2018/view/Default.aspx.

3. Heaf J. Current trends in European renal epidemiology. Clin Kidney J. 2017;10(2):149-153.

4. Saini T, Murtagh FE, Dupont PJ, McKinnon PM, Hatfield P, Saunders Y. Comparative pilot study of symptoms and quality of life in cancer patients and patients with end stage renal disease. Palliative medicine. 2006;20(6):631-636.

5. Bossola M, Vulpio C, Tazza L. Fatigue in chronic dialysis patients. Seminars in dialysis. 2011;24(5): 550-555.

6. Abdel-Kader K, Unruh ML, Weisbord SD. Symptom burden, depression, and quality of life in chronic and end-stage kidney disease. Clinical journal of the American Society of Nephrology : CJASN. 2009;4(6):1057-1064.

7. Davison SN, Jhangri GS. Impact of pain and symptom burden on the health-related quality of life of hemodialysis patients. J Pain Symptom Manage. 2010;39(3):477-485.

8. Riley WT, Rothrock N, Bruce B, et al. Patient-reported outcomes measurement information system (PROMIS) domain names and definitions revisions: further evaluation of content validity in IRTderived item banks. Qual Life Res. 2010;19(9):1311-1321.

9. Dantzer R, Heijnen CJ, Kavelaars A, Laye S, Capuron L. The neuroimmune basis of fatigue. Trends Neurosci. 2014;37(1):39-46.

10. Hardy SE, Studenski SA. Qualities of fatigue and associated chronic conditions among older adults. $J$ Pain Symptom Manage. 2010;39(6):1033-1042.

11. Bossola M, Di Stasio E, Antocicco M, Tazza L. Qualities of fatigue in patients on chronic hemodialysis. Hemodialysis international. International Symposium on Home Hemodialysis. 2013;17(1):32-40.

12. Sakkas GK, Karatzaferi C. Hemodialysis fatigue: just "simple" fatigue or a syndrome on its own right? Frontiers in physiology. 2012;3:306.

13. Ju A, Unruh ML, Davison SN, et al. Patient-Reported Outcome Measures for Fatigue in Patients on Hemodialysis: A Systematic Review. American journal of kidney diseases : the official journal of the National Kidney Foundation. 2018;71(3):327-343.

14. Abdel-Kader K, Jhamb M, Mandich LA, et al. Ecological momentary assessment of fatigue, sleepiness, and exhaustion in ESKD. BMC nephrology. 2014;15:29.

15. Riis J, Loewenstein G, Baron J, Jepson C, Fagerlin A, Ubel PA. Ignorance of hedonic adaptation to hemodialysis: a study using ecological momentary assessment. Journal of experimental psychology. General. 2005;134(1):3-9.

16. Caplin B, Kumar S, Davenport A. Patients' perspective of haemodialysis-associated symptoms. Nephrology, dialysis, transplantation : official publication of the European Dialysis and Transplant Association - European Renal Association. 2011;26(8):2656-2663.

17. Horigan AE, Barroso JV. A Comparison of Temporal Patterns of Fatigue in Patients on Hemodialysis. Nephrology nursing journal : journal of the American Nephrology Nurses' Association. 2016;43(2):129138, 148; quiz 139.

18. Jhamb M, Weisbord SD, Steel JL, Unruh M. Fatigue in patients receiving maintenance dialysis: a review of definitions, measures, and contributing factors. American journal of kidney diseases : the official journal of the National Kidney Foundation. 2008;52(2):353-365.

19. Picariello F, Moss-Morris R, Macdougall IC, Chilcot AJ. The role of psychological factors in fatigue among end-stage kidney disease patients: a critical review. Clin Kidney J. 2017;10(1):79-88. 
20. Bossola M, Luciani G, Tazza L. Fatigue and its correlates in chronic hemodialysis patients. Blood purification. 2009;28(3):245-252.

21. Bossola M, Di Stasio E, Giungi S, Rosa F, Tazza L. Fatigue is associated with serum interleukin- 6 levels and symptoms of depression in patients on chronic hemodialysis. J Pain Symptom Manage. 2015;49(3):578-585.

22. De Houwer J. The propositional approach to associative learning as an alternative for association formation models. Learning \& behavior. 2009;37(1):1-20.

23. Bovbjerg DH, Montgomery GH, Raptis G. Evidence for classically conditioned fatigue responses in patients receiving chemotherapy treatment for breast cancer. Journal of behavioral medicine. 2005;28(3):231-237.

24. Lenaert $B$, Boddez $Y$, Vlaeyen JWS, van Heugten $C M$. Learning to feel tired: A learning trajectory towards chronic fatigue. Behaviour research and therapy. 2018;100:54-66.

25. Craven JL, Rodin GM, Littlefield C. The Beck Depression Inventory as a screening device for major depression in renal dialysis patients. International journal of psychiatry in medicine. 1988;18(4): 365-374.

26. Krupp LB, LaRocca NG, Muir-Nash J, Steinberg AD. The fatigue severity scale. Application to patients with multiple sclerosis and systemic lupus erythematosus. Archives of neurology. 1989;46(10): 1121-1123.

27. Artom M, Moss-Morris R, Caskey F, Chilcot J. Fatigue in advanced kidney disease. Kidney international. 2014;86(3):497-505.

28. Di lorio B, Cillo N, Cirillo M, De Santo NG. Charlson Comorbidity Index is a predictor of outcomes in incident hemodialysis patients and correlates with phase angle and hospitalization. The International journal of artificial organs. 2004;27(4):330-336.

29. Katz S, Ford AB, Moskowitz RW, Jackson BA, Jaffe MW. STUDIES OF ILLNESS IN THE AGED. THE INDEX OF ADL: A STANDARDIZED MEASURE OF BIOLOGICAL AND PSYCHOSOCIAL FUNCTION. Jama. 1963;185:914-919.

30. Lindsay RM, Heidenheim PA, Nesrallah G, Garg AX, Suri R, Daily Hemodialysis Study Group London Health Sciences $C$. Minutes to recovery after a hemodialysis session: a simple health-related quality of life question that is reliable, valid, and sensitive to change. Clinical journal of the American Society of Nephrology : CJASN. 2006;1(5):952-959.

31. Smarr KL, Keefer AL. Measures of depression and depressive symptoms: Beck Depression Inventory-II (BDI-II), Center for Epidemiologic Studies Depression Scale (CES-D), Geriatric Depression Scale (GDS), Hospital Anxiety and Depression Scale (HADS), and Patient Health Questionnaire-9 (PHQ-9). Arthritis care \& research. 2011;63 Suppl 11:S454-466.

32. Sohl SJ, Friedberg F. Memory for fatigue in chronic fatigue syndrome: relationships to fatigue variability, catastrophizing, and negative affect. Behavioral medicine (Washington, D.C.). 2008;34(1):29-38.

33. Ju A, Unruh M, Davison S, et al. Establishing a Core Outcome Measure for Fatigue in Patients on Hemodialysis: A Standardized Outcomes in Nephrology-Hemodialysis (SONG-HD) Consensus Workshop Report. American journal of kidney diseases : the official journal of the National Kidney Foundation. 2018;72(1):104-112.

34. Sklar AH, Riesenberg LA, Silber AK, Ahmed W, Ali A. Postdialysis fatigue. American journal of kidney diseases : the official journal of the National Kidney Foundation. 1996;28(5):732-736.

35. Cardenas DD, Kutner NG. The problem of fatigue in dialysis patients. Nephron. 1982;30(4):336-340.

36. de Ridder D, Geenen R, Kuijer R, van Middendorp H. Psychological adjustment to chronic disease. Lancet (London, England). 2008;372(9634):246-255.

37. Stewart-Williams S, Podd J. The placebo effect: dissolving the expectancy versus conditioning debate. Psychological bulletin. 2004;130(2):324-340. 



\section{Chapter 5}

\section{Daily physical activity in patients on chronic haemodialysis and its relation to fatigue and depressive symptoms}

Brys ADH, Bossola M, Lenaert B, Biamonte F, Gambaro G, Di Stasio E Published in Int Urol Nephrol. 2020;52(10):1959-1967 


\section{Abstract}

\section{Objective}

Fatigue and depressed mood are considered main impediments to physical activity in haemodialysis (HD) patients. A better understanding of their interrelationships is crucial in order to develop effective therapies. Moreover, measurement of daily physical activity (DPA) in HD patients is tricky, as it is usually assessed by subjective self-report questionnaires. Therefore, we aimed to objectively measure spontaneous DPA with motion sensors and to explore its relation with fatigue and depressive symptoms.

\section{Methods}

DPA was assessed for 7 consecutive days in 37 HD patients based on their daily step count measured with the SenseWear ${ }^{\mathrm{TM}}$ Armband. The Fatigue Severity Scale (FSS) and Beck Depression Inventory-II (BDI-II) were administered to evaluate fatigue and depressed mood.

\section{Results}

Median DPA was 2424 steps/day, [IQR:892-4545]. In 81\% of subjects, DPA felt within a sedentary lifestyle classification, as they made $<5.000$ steps/day. DPA did not correlate with fatigue $\left(r_{s}=0.04, p=0.832\right)$, and did not significantly differ between patients categorized as clinically fatigued $(n=23, F S S \geq 4)$ or not $(n=14, F S S<4)(p=0.654$, $d=0.20)$. Although low-depressed subjects $(n=19$, BDI-II $\leq 13)$ made on average 1.7 times more steps/day than high-depressed subjects ( $n=18, B D I-\|>13) \quad(p=0.111$, $d=0.60)$, depressive mood did also not correlate significantly with DPA $\left(r_{s}=-0.23\right.$, $p=0.175)$.

\section{Conclusion}

Objective assessment of DPA with motion sensors is feasible in HD patients and allows identifying a sedentary lifestyle. Our results suggest spontaneous DPA is determined by age rather than by fatigue or mood. 


\section{Introduction}

Patients undergoing chronic haemodialysis (HD) are less active than sedentary healthy controls. ${ }^{1}$ Decreased physical activity in HD patients may be the consequence of multiple factors such as the effect of uraemia on muscle function causing atrophy, anaemia, bone disease, malnutrition, comorbid conditions or the dialysis procedure itself, since many patients complain of fatigue following the HD treatment. Indeed, according to HD patients, fatigue and depressed mood are among the main impediments to physical exercise. ${ }^{2}$ Interestingly, physical exercise in turn may reduce fatigue severity and depression. ${ }^{3}$ However, it is less clear to what extent fatigue and depressed mood are related to spontaneous daily physical activity (DPA) (i.e. daily step count) in HD patients. Since fatigue, depression and physical inactivity are all associated with increased mortality risk and reduced health-related quality of life (HRQoL) in renal patients, a better understanding of their interrelationships is crucial in order to develop effective therapies, improve HD patients' HRQOL and reduce the associated mortality risk. ${ }^{4,5}$

Fatigue is one of the most common and tedious symptoms among HD patients with a prevalence of $40-80 \% .{ }^{6,7}$ It is a complex phenomenon and may be determined by multiple factors including biological (e.g. inflammation), psychological (e.g. depression or anxiety), social (e.g. poor social support) and behavioural (e.g. sleep or physical activity) variables. ${ }^{8-12}$ However, apart from a consistently reported significant association with depressive symptoms, research findings are largely inconsistent and no causal relationship has yet been demonstrated. ${ }^{13}$

A recently published meta-analysis showed that $23-39 \%$ of HD patients suffer from depression, according to the methodology of diagnosis (i.e. interview-based versus self- or clinician-administered rating scales). ${ }^{14}$ Likewise fatigue, depression in HD patients may result from a complex interaction of biological, psychological and social factors. ${ }^{15,16}$ For instance, constraints associated with the dialysis treatment such as inability to work or reduced possibilities to participate in social activities may affect self-esteem and may trigger depressive feelings. ${ }^{17}$

Assessing DPA in HD patients in a reliable and valid way is challenging. Indeed, DPA is often assessed by administering self-report questionnaires or diaries. ${ }^{5,18-20}$ These 
subjective measurement instruments are easy to administer but may be liable to memory recall bias or inaccurate perception of activity behaviour. ${ }^{21}$ In contrast, step counting and energy expenditure are objective outcome measures to assess DPA and can be measured in daily life through the application of wearable motion sensors (i.e. pedometers, accelometers or multisensors). ${ }^{22}$ These devices have gained growing interest as a method to evaluate DPA in patients with chronic diseases like chronic obstructive pulmonary disease and chronic heart failure. ${ }^{23,24} \mathrm{~A}$ number of studies reported on the use of a motion sensor to estimate DPA in HD patients. ${ }^{1,4,25-30}$ However, the majority of these reports have only assessed demographical and clinical correlates of DPA in HD patients (e.g. age, gender, body mass index, haemoglobin - , serum albumin - and serum creatinine levels).

Despite the high prevalence of fatigue and depressive symptoms in HD patients, their relationship with objectively measured DPA has received little research attention. ${ }^{4,26,30}$ In contrast, their association with intradialytic physical exercise (aerobic, resistance or both) or functional capacity (e.g. 6-min walk test or sit-to-stand and stair-climbing tests), is more frequently investigated and used as a reflection of HD patients' physical activity. ${ }^{31-33}$ These reports showed negative associations between both fatigue and depression and physical activity.

However, the few reports investigating DPA and fatigue or depression are somewhat inconsistent. For instance, Zhang et al. ${ }^{4}$ reported a negative association between depressed mood, assessed through the Beck Depression Inventory (BDI), and HD patients' DPA, measured with a body activity monitor. However, Sheshadri et al. ${ }^{26}$ did not find any association with depressive symptoms, administering the Center for Epidemiologic Studies - Depression Scale (CES-D). They also found that higher DPA, measured with pedometers recording daily steps, was associated with lower fatigue scores. ${ }^{26}$ However, fatigue was measured using the Dialysis Symptom Index (DSI) questionnaire and the Kidney Disease Quality of Life Vitality Scale (KDQOL-VS), which are not fatigue-specific questionnaires.

Therefore, we aimed (i) to objectively measure HD patients' DPA using a wearable motion sensor (SenseWear ${ }^{\mathrm{TM}}$ Armband) recording daily step count, and (ii) to investigate the relationship with fatigue and depressive symptoms, administering standardized measurement instruments (FSS and BDI, respectively). 


\section{Subjects and methods}

\section{Study subjects}

All prevalent patients of the HD unit of the Catholic University of Rome (Italy) on a chronic HD treatment for at least six months between June 2018 and May 2019 were considered eligible for this observational study. Exclusion criteria were as follow: $<18$ years of age, diagnosed neuromuscular disease, wheelchair dependence or amputations in lower extremities preventing to walk, inability to answer the questionnaires because of hearing or reading problems, dementia, actual instability of clinical condition requiring hospitalization. All subjects gave informed consent for study participation and the study was approved by the local ethics committee (protocol number 13615/18 - 22147/18).

\section{Study measurements}

\section{Assessment of DPA}

To measure DPA, The SenseWear ${ }^{\mathrm{TM}}$ Armband (SWA; BodyMedia, Pittsburgh, PA, USA) was used. The SWA is a personalized multisensory body device comprised of a heatflux sensor, a skin-temperature sensor, a near body ambient temperature sensor, a galvanic skin response sensor and a two-axis accelerometer, providing information about body position (lying or not), and enables continuous physiological monitoring. ${ }^{22}$ Furthermore, the SWA records step count at 1-min intervals. Collected data are exported via the Professional InnerView Software 7.0 (Body Media Inc., Pittsburgh, PA). ${ }^{22}$ The software recognizes daily activities such as walking, running, biking, resting or sleeping, and computes number of daily steps, activity-related energy expenditure and physical activity level. ${ }^{22}$ The validity of the device has been tested in clinical studies with patients suffering from coronary heart disease, chronic obstructive pulmonary disease, arthritis and stroke. ${ }^{34}$ The SWA was attached around the opposite upper arm of the arterial venous fistula at the level of the posterior triceps. Patients were instructed to wear the device for seven consecutive days, except when it would become wet, such as during bathing or swimming. Patients who wore the SWA all seven study days for at least eight hours a day of which at least six hours in the upright position (i.e. sitting, standing or walking) were included in the analysis. From 
the raw data, the mean number of steps per separate day throughout the entire week of recording was obtained.

Assessment of fatigue and depressive symptoms

Subjects completed the Beck Depression Inventory-II (BDI-II) and Fatigue Severity Scale (FSS). The BDI-II is a patient-rated 21-item inventory to evaluate depressive symptoms during the previous two weeks and has been validated in the HD population. Scores can range from 0 to 63 . BDI-II classification was as follows: 0-13: no depression; 14-19: mild depression; 20-28: moderate depression; 29-63: severe depression. ${ }^{35}$ The FSS is a nine-item questionnaire assessing fatigue severity in daily life and uses a seven-point Likert scale. The total score is calculated by averaging the scores of the individual item responses. Individual item scores can range from 1-7 (nomaximum fatigue). A total FSS score of four or more is considered as indicative of problematic fatigue. ${ }^{36}$ According to a systematic review on fatigue instruments used in haemodialysis, the FSS is the most frequently used measurement instrument to evaluate fatigue in renal patients. ${ }^{37}$

\section{Baseline and clinical data}

The following demographical, clinical, and laboratory information was obtained from medical records: age, gender, dialysis vintage, primary cause of ESKD, type and number of comorbidities assessed by the Charlson Comorbidity Index, self-reported functional status via the activity of daily living (ADL) scale, Body Massa Index (BMI), haemoglobin, sodium, calcium, phosphorus, albumin, PTH, creatinine, and dialysis dose as evaluated by $K t / \mathrm{V}$.

\section{Haemodialysis treatment}

Patients received bicarbonate haemodialysis treatment between 3.5 and 4 hours three times weekly. The blood flow ranged from 250 to $300 \mathrm{ml} / \mathrm{minute}$ with a dialysis rate flow of $500 \mathrm{ml} /$ minute. High permeability membranes were used.

\section{Procedure}

After giving their informed consent, each participant received the SWA along with verbal instructions from the attending physician during a regular dialysis session. Questionnaires were completed during the HD session at the end of the study week. 
Demographical and clinical information was obtained at the moment of inclusion. Results of monthly routine biochemical and haematological investigation were obtained closest to the day on which the study started.

\section{Statistical analysis}

Statistical analysis was performed by using the Statistical Package for Social Science (SPSS). The Shapiro-Wilk test showed that the assumption for normality of distribution was violated for DPA and mood. Therefore, non-parametric tests were computed. Continuous variables are expressed as mean and standard deviation (SD) or median and [range] as appropriate and categorical variables displayed as frequencies. To evaluate the difference in DPA across the different measurement days, a nonparametric Friedman ANOVA was run. In order to investigate correlations between DPA, fatigue and depressive symptoms, Spearman's rho correlation coefficients $\left(r_{s}\right)$ were calculated. Additionally, we evaluated differences in DPA between patient subgroups (i.e. fatigued versus non-fatigued and patients low or high in depressive symptoms) using non-parametric Mann-Whitney $U$ tests. To study the relationship between DPA and covariates (i.e. fatigue, mood, age, gender, number of comorbidities and serum albumin), a multiple linear regression analysis with a backward-stepwise method was performed. Adjusted $R$-squared $\left(R^{2}\right)$ values measured the proportion of variability explained by the model. Statistical significance was established at $p$-values $<0.05$.

\section{Results}

\section{Participant characteristics}

Fifty-four patients were approached of whom 51 provided informed consent $(94 \%$ consent rate). One subject discontinued the study because of a skin rash due to the SWA, another subject discontinued the study because of hospitalization for kidney transplantation, and another one withdrew due to transfer to another dialysis center. The remaining 48 individuals with a mean age of 63 years $(S D=18)$ are included in this report. Eleven subjects were excluded from the analyses involving DPA because of incomplete data due to a setting error of the SWA resulting in a battery runtime of 
only three instead of seven days. Baseline clinical, demographical and laboratory characteristics as well as BDI-II and FSS scores are reported in Table 5.1.

Table 5.1 Baseline demographical, clinical and laboratory characteristics $(n=48)$.

\begin{tabular}{lc}
\hline Characteristic & Mean \pm SD [range] / $\mathbf{n}$ (percentage) \\
\hline Age (years) & $63 \pm 18[20-88]$ \\
Sex (male) & $28(58 \%)$ \\
Body Mass Index (kg/m ${ }^{2}$ ) & $24.0 \pm 4.4[17.5-37.7]$ \\
Dialysis vintage, months & $51 \pm 54[6-216]$ \\
Primary cause of ESRD & \\
$\quad$ hypertension & $14(29 \%)$ \\
glomerulonephritis & $7(15 \%)$ \\
diabetes & $14(29 \%)$ \\
interstitial nephritis & $3(6 \%)$ \\
polycystic kidney disease & $6(13 \%)$ \\
$\quad$ others/unknown & $4(8 \%)$ \\
Charlson Comorbidity Index & $2 \pm 2[0-6]$ \\
Activity of Daily Living & $5 \pm 1[1-6]$ \\
Kt/V: urea clearance & $1.5 \pm 0.3$ \\
Interdialytic weight gain (kg) & $1.9 \pm 0.7$ \\
Haemoglobin (g/dl) & $10.4 \pm 1.0$ \\
Serum Creatinine (mg/dl) & $10.0 \pm 2.6$ \\
Calcium (mg/dl) & $9.2 \pm 0.7$ \\
Serum Sodium (mmol/l) & $139 \pm 3$ \\
Dialysate Sodium (mmol/l) & $139 \pm 2$ \\
Parathyroid hormone (pg/ml) & $565 \pm 561$ \\
Phosphorus (mg/dl) & $5.2 \pm 1.5$ \\
Albuminemia (g/dl) & $3.5 \pm 0.3$ \\
Beck Depression Inventory-Il score & $15 \pm 10[2-46]$ \\
Fatigue Severity Scale score & $4.0 \pm 1.6[1.3-6.7]$ \\
Step count/day (median [IQR]) & $2424[892-4545]$ \\
\hline
\end{tabular}

\section{Daily physical activity}

Thirty-seven participants wore the SWA daily, with an average of 21.8 hours/day. The median daily step count was 2424 (IQR: 892-4545). Thirty individuals (81\%) made less than 5.000 steps/day matching with a sedentary lifestyle according to Tudor-Locke et al.. $^{38}$ Similarly, the SWA software algorithm categorized patients' physical activity in $95 \pm 6 \%$ (range $70-100 \%$ ) and $5 \pm 5 \%$ (range $0-30 \%$ ) of all cases as a sedentary and moderate intensity level, respectively.

Figure 5.1 illustrates participants' DPA, by means of daily step count, for each of the seven measurement days. Friedman ANOVA analysis with daily step count as the dependent variable revealed a significant difference across measurement days, 
$\chi^{2}(6)=14.33, p=0.026$. Paired samples $t$-test revealed that participants took on average 2589 ( $\mathrm{SE}=426$ ) steps/day on treatment days versus 3485 ( $\mathrm{SE}=524)$ steps/day on nontreatment days. This difference of -897 steps, BCa $95 \% \mathrm{Cl}[-1629,-255]$, was significant, $t(36)=-2.72, p=0.010$, and represented an effect size of $d=0.28$.

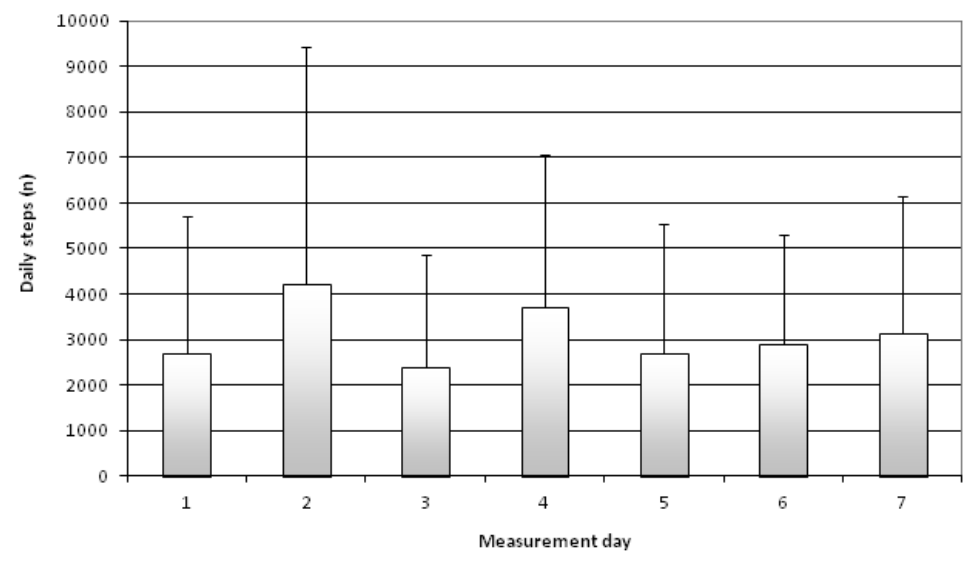

Figure 5.1 Haemodialysis (HD) patients' mean daily step count for each measurement day. Patients underwent their regular HD on day 1,3 and 5 . $(n=37)$ Median daily step count was 2424 steps/ day [IQR: 892-4545].

\section{DPA and its relation to demographical and clinical variables}

Correlations between DPA and demographical and clinical variables are reported in Table 5.2. Results showed a negative correlation between DPA and age, $r_{s}=-0.62$, $p<0.001$, and DPA and sleep time (hours of sleep registered by the SWA), $r_{s}=-0.37$, $p=0.023$. A positive correlation was found between DPA and albuminemia $\left(r_{s}=0.45\right.$, $p=0.005)$, and DPA and serum creatinine levels $\left(r_{s}=0.39, p=0.016\right)$.

\section{DPA and its relation to fatigue and depressive symptoms}

DPA did not significantly correlate with FSS- or BDI-II scores $\left(r_{s}=0.04, p=0.832\right.$; and $r_{s}=-0.23, p=0.175$, respectively). To test whether DPA varied between individuals suffering from fatigue or depressive mood, respectively, participants were subdivided based on the clinical cut-off scores used for the FSS and BDI-II. This resulted into a 'high fatigue' ( $n=23$, FSS score $\geq 4$ ) and 'low fatigue' ( $n=14, F S S<4)$ subgroup, and participants 'low' ( $n=19$, BDI-II score $\leq 13$ ) or 'high' in depressive symptoms ( $n=18$, 
BDI-II >13). Figure 2 shows the mean daily step count, as function of the measurement day, for these subgroups (panel $a$ and $b$, respectively). Their corresponding medians and ranges, $p$-values and effect sizes are reported in Table 5.3A and 5.3B.

Table 5.2 Univariate correlation analysis between daily physical activity (DPA), by means of daily step count, and demographical and clinical variables (Spearman's rho coefficient) ( $n=37)$.

\begin{tabular}{lcccccccc}
\hline & $\begin{array}{c}\text { Age } \\
\text { (years) }\end{array}$ & $\begin{array}{c}\text { Gender } \\
(\mathbf{M} / \mathbf{F})\end{array}$ & $\mathbf{C C l}$ & $\begin{array}{c}\mathrm{BMI} \\
\left(\mathbf{k g} / \mathbf{m}^{2}\right)\end{array}$ & $\begin{array}{c}\mathrm{Hb} \\
(\mathrm{g} / \mathrm{dl})\end{array}$ & $\begin{array}{c}\text { Creatinine } \\
(\mathbf{m g} / \mathbf{d l})\end{array}$ & $\begin{array}{c}\text { Albumin } \\
(\mathbf{g} / \mathbf{d l})\end{array}$ & $\begin{array}{c}\text { Sleep time } \\
\text { (hours) }\end{array}$ \\
\hline DPA & -0.62 & 0.16 & -0.27 & -0.10 & 0.03 & 0.39 & 0.45 & -0.37 \\
$p$-value & $<0.001$ & 0.334 & 0.108 & 0.539 & 0.882 & 0.016 & 0.005 & 0.023 \\
\hline
\end{tabular}

$\mathrm{CCl}$ : Charlson Comorbidity Index, BMI: Body Mass Index, $\mathrm{Hb}$ : haemoglobin, serum creatinine, serum albumin, Sleep time: hours of sleep registered by the SenseWear ${ }^{\mathrm{TM}}$ Armband

Table 5.3A shows that there were no significant differences in DPA between low- and high-fatigued subgroups through all different measurement days. $P$-values range from 0.231 to 0.938 with very small to small effect sizes $(d<0.20-0.47)$. The mean ratio of mean daily step count between low FSS and high FSS subgroups is 1.2, and ranges from 1.0 to 1.5 among the different measurement days ( $p=0.654, d=0.20)$. Neither was a significant correlation found between fatigue and mean daily step count on treatment versus non-treatment days, $r_{s}=0.05, p=0.759$ and $r_{s}=-0.09, p=0.595$; respectively.

Table 5.3B shows the levels of DPA for low- and high-depressed individuals. Here, there only was a significant difference in daily step count on the second measurement day, which was a non-treatment day, representing a medium effect size $(d=0.61)$. No significant differences were found on other days. Over the entire study week, the ratio of mean daily step count between low- and high-depressed participants ranges from 1.3 to 2.3 among separate measurement days, with a mean ratio of $1.7,(p=0.111$, $d=0.60$ ). Indeed, low-depressed participants took on average almost twice as many steps/day in contrast to their high-depressed counterparts. However, these differences were not statistically significant, probably because of the large spread of daily step count between participants in this study sample. Neither was a significant correlation found between depressive mood and mean daily step count on treatment versus non-treatment days, $r_{s}=-0.20, p=0.226$ and $r_{s}=-0.23, p=0.168$; respectively. 

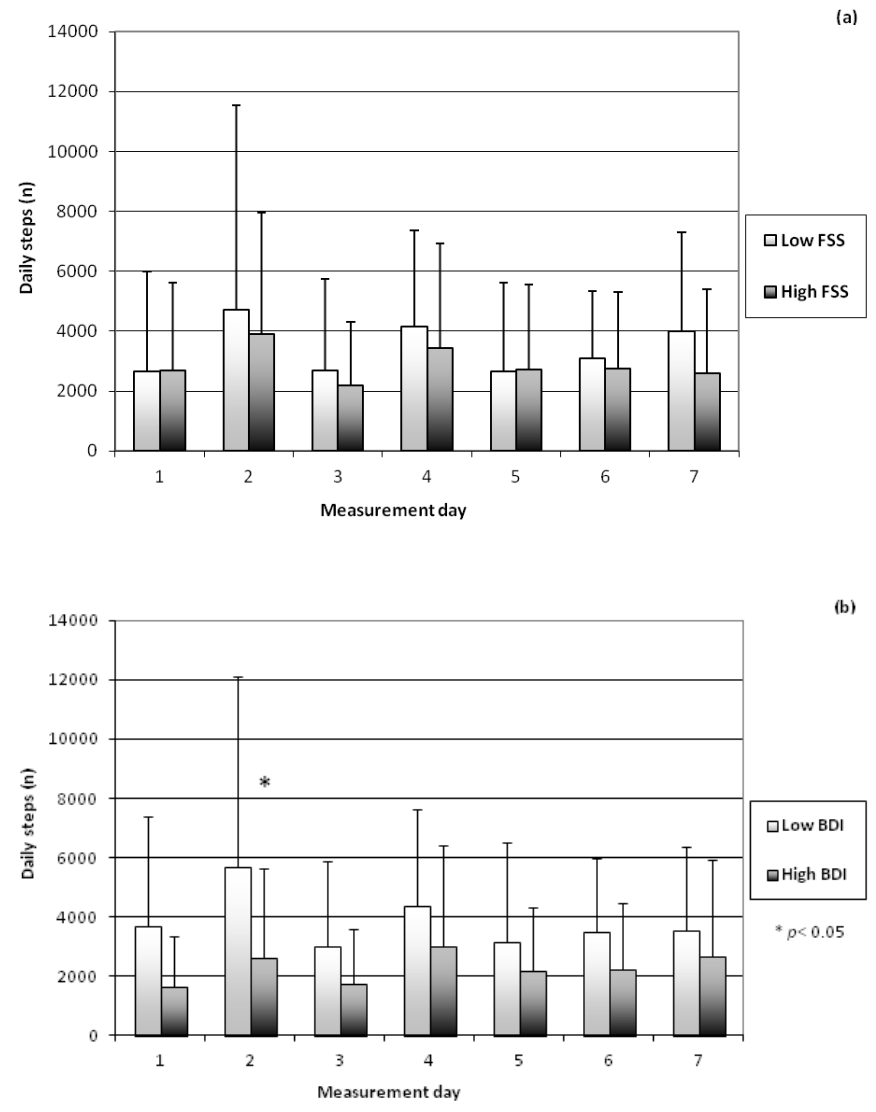

Figure 5.2 HD patients' daily step count subdivided for reported levels of fatigue (a) and depressed symptoms (b). FSS: Fatigue Severity Scale (low <4, high $\geq 4$ ); BDI: Beck Depression Inventory-II (low $\leq 13$, high $>13$ ). Patients underwent their regular haemodialysis treatment on day 1,3 and $5 .{ }^{*} p<0.05(\mathrm{n}=37)$.

Finally, a correlation matrix between FSS- and BDI questionnaire scores and demographical and clinical variables is reported in Supplemental Table S5.1, showing fatigue and depressed mood, and age and albuminemia are significantly correlated $\left(r_{s}=0.34, p=0.020\right.$ and $r_{s}=0.39, p=0.017$; respectively). Furthermore, fatigue and depressed mood did not significantly correlate with sleep time. However, the time patients spent resting (i.e. supine position registered by the SWA) significantly and positively correlated with depressed $\operatorname{mood}\left(r_{s}=0.45, p=0.006\right)$ but not with fatigue $\left(r_{s}=0.32, p=0.056\right)$. 
Table 5.3 Daily physical activity, by means of daily step count, in haemodialysis patients subdivided for fatigue: low (FSS <4) or high (FSS $\geq 4)(A)$; and depressive symptoms: low (BDI-II $\leq 13$ ) or high $(B D I-I I>13)(B)$, as function of the measurement day $(n=37)$.

\begin{tabular}{lcccc}
\hline A - Measurement day & $\begin{array}{c}\text { Low FSS }(\mathbf{n}=\mathbf{1 4}) \\
\text { Median [range] }\end{array}$ & $\begin{array}{c}\text { High FSS }(\mathbf{n}=\mathbf{2 3}) \\
\text { Median [range] }\end{array}$ & $\boldsymbol{p}$ & Effect size (Cohen's $\boldsymbol{d}$ ) \\
\hline $1^{*}$ & $1371[101-10121]$ & $1793[49-11095]$ & 0.793 & $<0.20$ - very small \\
2 & $2051[15-26059]$ & $1909[0-14588]$ & 0.817 & $<0.20$ - very small \\
$3^{*}$ & $1390[168-11223]$ & $1953[17-9177]$ & 0.817 & $<0.20$ - very small \\
4 & $3939[9-10337]$ & $2213[3-12427]$ & 0.411 & 0.21 - small \\
$5^{*}$ & $1863[124-10848]$ & $1654[10-10956]$ & 0.938 & $<0.20$ - very small \\
6 & $3276[33-8203]$ & $2219[14-8042]$ & 0.429 & $<0.20$ - very small \\
7 & $3640[21-10830]$ & $1885[6-9436]$ & 0.745 & 0.47 - small \\
\hline B - Measurement day & Low BDI-II (n=19) & High BD-II (n=18) & $\boldsymbol{p}$ & Effect size (Cohen's $\boldsymbol{d}$ ) \\
& Median [range] & Median [range] & & \\
\hline $1^{*}$ & $2053[61-11095]$ & $1091[49-6427]$ & 0.066 & 0.72 - medium \\
2 & $4612[42-26059]$ & $1179[0-9899]$ & 0.049 & 0.61 - medium \\
$3^{*}$ & $1953[28-11223]$ & $1088[17-6497]$ & 0.105 & 0.52 - medium \\
4 & $3978[31-11263]$ & $1790[3-12427]$ & 0.150 & 0.40 - small \\
$5^{*}$ & $2142[58-10956]$ & $1257[10-6041]$ & 0.499 & 0.34 - small \\
6 & $3209[24-8203]$ & $1619[14-8042]$ & 0.105 & 0.53 - medium \\
7 & $3118[36-8482]$ & $1663[6-10830]$ & 0.233 & 0.29 - small \\
\hline
\end{tabular}

*Haemodialysis treatment days, FSS: Fatigue Severity Scale, BDI-II: Beck Depression Inventory-II

\section{Predictor variables for DPA in HD patients}

Finally, a multiple linear regression analysis was carried out with mean daily step count as the dependent variable and with fatigue, depressive symptoms, age, gender, $\mathrm{CCl}$ and serum albumin level as covariates (Table 5.4). Using a backward-stepwise approach, only age remained as independent significant predictor for DPA in the final model $(p=0.004)$.

Table 5.4 Results of the multiple linear regression analysis with daily step count as the dependent variable $(n=37)$.

\begin{tabular}{|c|c|c|c|}
\hline \multicolumn{4}{|c|}{ Entire regression model: adjusted $\mathrm{R}^{2}=0.13$} \\
\hline Covariate & Correlation coefficients (standardized beta) & Standard Error & $\boldsymbol{p}$ \\
\hline FSS & 0.07 & 2197.30 & 0.705 \\
\hline BDI-II & -0.11 & 356.00 & 0.562 \\
\hline Age (years) & -0.31 & 210.96 & 0.132 \\
\hline $\mathrm{CCl}$ & -0.14 & 2270.27 & 0.440 \\
\hline Gender (female/male) & 0.11 & 6992.94 & 0.534 \\
\hline Albuminemia & 0.19 & 11664.25 & 0.297 \\
\hline \multicolumn{4}{|c|}{ Reduced regression model (backward-stepwise method): adjusted $R^{2}=0.20$} \\
\hline Covariate & Correlation coefficients (standardized beta) & Standard Error & $p$ \\
\hline Age (years) & -0.47 & 160.17 & 0.004 \\
\hline
\end{tabular}

FSS: Fatigue Severity Scale, BDI-II: Beck Depression Inventory-II, CCI: Charlson Comorbidity Index 


\section{Discussion}

The present study objectively examined HD patients' spontaneous physical activity in daily life based on daily step count measured with a wearable motion sensor. Furthermore, we assessed the relation between DPA and subjectively reported symptoms of fatigue and depressive mood. ${ }^{2}$

First, results showed that in $81 \%$ of the HD patients, DPA can be classified as sedentary as they made less than 5.000 steps a day. ${ }^{38}$ These findings are in accordance with previous reports showing that HD patients' DPA is often lower than healthy sedentary control subjects. ${ }^{1,25,26}$ However, the distribution of daily steps taken by these HD patients (Figure 5.1) also shows that DPA considerably varies between individuals. Indeed, some HD patients take a reasonable number of steps per day, whereas others hardly undertake any spontaneous physical activity in daily life. The benefits of an active lifestyle, such as decreased risks for obesity, hypertension, cardiovascular disease, diabetes and all-cause mortality are especially relevant to HD patients. The use of motion sensors can help to objectively identify patients with a sedentary lifestyle at risk of the aforementioned health problems. Furthermore, it might become a useful tool to support patients in improving their DPA, and consequently their health and HRQoL., ${ }^{5,39-42}$

Results showed a significant difference in DPA on treatment versus non-treatment days. This may partially be explained by the fact that patients are forced into a prolonged sitting position during the dialysis session. Additionally, there might be other factors contributing to this difference, for instance treatment related fatigue leading to decreased physical activity during the rest of the day.

In our study sample, DPA was not related to individuals' general fatigue experience $\left(r_{s}=-0.04\right)$. This result is in contrast to a previous report of Sheshadri et al. ${ }^{26}$ who found a negative correlation between objectively measured daily step count and fatigue severity in HD patients (adj. $r=-0.20, p=0.04$ ). However, Sheshadri et al. administered different measurement instruments to assess fatigue (i.e. DSI and KDQOL-VS), making it difficult to directly compare results. These questionnaires evaluate fatigue in only two and four items respectively, as a general feeling of being tired or having a lack of energy. In contrast, the FSS is a fatigue-specific questionnaire and might better 
capture the fatigue experience in daily life (e.g. whether fatigue interferes with one's physical functioning). Additionally, the study population of Sheshadri et al. was slightly younger with a mean age (IQR) of 57 years (52-65) versus 63 years (51-78) in our study sample. The older age of our study sample might have contributed to the lack of finding an association between fatigue and DPA. Indeed, a decline in DPA in older age is commonly observed and is likely determined by multiple factors, such as loss of muscle mass, nutritional state, pain symptoms, comorbidities; and may thus not only be the result of fatigue. In our multiple linear regression analysis, older age turned out to be the only independent predictor variable for lower DPA in this study sample, explaining $20 \%$ of its variance. Considering that the majority of the HD population belong to older age groups, efforts to improve these patients' activity levels are indispensable since they have much to gain from improved physical activity.

Furthermore, the finding that objectively measured DPA did not correlate with subjectively reported fatigue underlines the complexity of fatigue as there is no linear relationship between fatigue and exertion. Interestingly, this seems to be in line with a recently proposed biopsychosocial model of fatigue in HD patients. ${ }^{9}$ In this model, psychological variables and social constituents, such as depression, anxiety or poor social support, can per se perpetuate a vicious circle of fatigue experience, that might have been originally triggered by physiological imbalances due to renal failure. Indeed, many reports previously established a relationship between fatigue and depressive mood or anxiety in HD patients. ${ }^{9,43,44}$ In the present study, there also was a positive association between fatigue severity and depressed mood $\left(r_{s}=0.34, p=0.020\right.$; Supplemental Table S5.1).

Finally, our results showed that high-depressed participants took almost half as many daily steps than low-depressed individuals. Although not statistically significant, a negative relationship between depressive mood and DPA was found $\left(r_{s}=-0.23\right)$. This finding is in accordance with a study of Zhang et al. ${ }^{4}$ who found a significant inverse correlation between BDI-II and DPA measured with an activity monitor during one week in a larger study sample consisting of $72 \mathrm{HD}$ patients $(r=-0.33, p=0.010)$. In a recently published meta-analysis, the beneficial effect of exercise on reducing depression, and other health-related parameters, in HD patients has been established. ${ }^{3,32}$ Investigating whether improving individuals' psychological condition may help to improve DPA may be an interesting scope for future trials. 
This study adds to the existing literature by objectively measuring physical activity of HD patients in the context of daily life. However, our study has several limitations. First, the FSS questionnaire does not distinguish between physical or mental fatigue, which might have contributed to the lack of an association between fatigue and DPA. However, none of the current fatigue questionnaires tested in HD patients and available in a language other than English do so and the FSS has proven to be useful in HD patients. ${ }^{37}$ Second, whereas we used real-time objective assessment of DPA in this study, future studies could also investigate real-time fatigue and mood using momentary assessment procedures. A third limitation is the relative small number of patients, due to the drop-out and exclusion of some participants because of a technical error setting of the SWA, causing some of our analyses may have been underpowered. Finally, future studies investigating the relationship between DPA and fatigue or depressive mood in HD patients may benefit from the inclusion of tests to evaluate functional capacity, and collect information about sleep quality, sleep disorders such as restless legs syndrome, and health-related quality of life, as they have been shown to be associated with physical activity, fatigue and depressed mood.

In conclusion, objective assessment of DPA with motion sensors is feasible in HD patients and may help to identify those at risk of the consequences of a sedentary lifestyle. Especially in the elderly, there is much to be gained as DPA was inversely correlated to age. Subjective experiences such as fatigue and mood merit more attention in daily clinical practice as they are central in patients' daily life and are related to an increased mortality risk and reduced HRQoL. Evaluating the effect of improving HD patients' depressed mood on DPA might be interesting for future studies. 


\section{Supplemental material}

Table S5.1 Correlations between questionnaire scores and demographical and clinical variables [Spearman's rho coefficient - ( $p$-value)] $(n=48)$.

\begin{tabular}{|c|c|c|c|c|c|c|c|}
\hline & FSS & $\mathrm{CCl}$ & Age (years) & $\begin{array}{l}\text { Gender } \\
\text { (F/M) }\end{array}$ & $\begin{array}{l}\text { Albuminemia } \\
(\mathrm{g} / \mathrm{L})\end{array}$ & $\begin{array}{l}\text { Supine } \\
\text { Position* } \\
\text { (hours) }\end{array}$ & $\begin{array}{c}\text { Sleep } \\
\text { Time* } \\
\text { (hours) }\end{array}$ \\
\hline \multirow[t]{2}{*}{ BDI-II } & 0.34 & 0.05 & 0.24 & -0.27 & -0.02 & 0.45 & 0.21 \\
\hline & $(0.020)$ & $(0.728)$ & $(0.097)$ & $(0.060)$ & $(0.890)$ & $(0.006)$ & $(0.223)$ \\
\hline \multirow[t]{2}{*}{ FSS } & - & -0.08 & -0.04 & -0.25 & -0.16 & 0.32 & 0.24 \\
\hline & & $(0.574)$ & $(0.766)$ & $(0.082)$ & $(0.343)$ & $(0.056)$ & (0.151) \\
\hline \multirow[t]{2}{*}{$\mathrm{CCl}$} & - & - & 0.28 & 0.21 & -0.01 & - & - \\
\hline & & & $(0.052)$ & $(0.162)$ & $(0.978)$ & & \\
\hline \multirow[t]{2}{*}{ Age } & - & - & - & -0.07 & -0.39 & - & - \\
\hline & & & & $(0.643)$ & $(0.017)$ & & \\
\hline \multirow[t]{2}{*}{ Gender } & - & - & - & - & 0.20 & - & - \\
\hline & & & & & $(0.240)$ & & \\
\hline
\end{tabular}

BDI-II: Beck Depression Inventory-II, FSS: Fatigue Severity Scale, CCI: Charlson Comorbidity Index. * $\mathrm{n}=37$ (Eleven patients excluded because of a setting error of the SenseWear ${ }^{\mathrm{TM}}$ Amband resulting in only three instead of 7 measurement days.) 


\section{References}

1. Johansen $\mathrm{KL}$, Chertow $\mathrm{GM}, \mathrm{Ng} \mathrm{AV}$, et al. Physical activity levels in patients on hemodialysis and healthy sedentary controls. Kidney international. 2000;57(6):2564-2570.

2. Hannan M, Bronas UG. Barriers to exercise for patients with renal disease: an integrative review. Journal of nephrology. 2017;30(6):729-741.

3. Song YY, Hu RJ, Diao YS, Chen L, Jiang XL. Effects of Exercise Training on Restless Legs Syndrome, Depression, Sleep Quality, and Fatigue Among Hemodialysis Patients: A Systematic Review and Metaanalysis. J Pain Symptom Manage. 2018;55(4):1184-1195.

4. Zhang M, Kim JC, Li Y, et al. Relation between anxiety, depression, and physical activity and performance in maintenance hemodialysis patients. Journal of renal nutrition : the official journal of the Council on Renal Nutrition of the National Kidney Foundation. 2014;24(4):252-260.

5. Johansen KL, Kaysen GA, Dalrymple LS, et al. Association of physical activity with survival among ambulatory patients on dialysis: the Comprehensive Dialysis Study. Clinical journal of the American Society of Nephrology : CJASN. 2013;8(2):248-253.

6. Davison SN, Jhangri GS. Impact of pain and symptom burden on the health-related quality of life of hemodialysis patients. J Pain Symptom Manage. 2010;39(3):477-485.

7. Saini T, Murtagh FE, Dupont PJ, McKinnon PM, Hatfield P, Saunders Y. Comparative pilot study of symptoms and quality of life in cancer patients and patients with end stage renal disease. Palliative medicine. 2006;20(6):631-636.

8. Dantzer R, Heijnen CJ, Kavelaars A, Laye S, Capuron L. The neuroimmune basis of fatigue. Trends Neurosci. 2014;37(1):39-46.

9. Picariello F, Moss-Morris R, Macdougall IC, Chilcot AJ. The role of psychological factors in fatigue among end-stage kidney disease patients: a critical review. Clin Kidney J. 2017;10(1):79-88.

10. Merlino G, Piani A, Dolso $P$, et al. Sleep disorders in patients with end-stage renal disease undergoing dialysis therapy. Nephrology, dialysis, transplantation : official publication of the European Dialysis and Transplant Association - European Renal Association. 2006;21(1):184-190.

11. Sabbatini M, Crispo A, Pisani A, et al. Sleep quality in renal transplant patients: a never investigated problem. Nephrology, dialysis, transplantation : official publication of the European Dialysis and Transplant Association - European Renal Association. 2005;20(1):194-198.

12. Jhamb M, Weisbord SD, Steel JL, Unruh M. Fatigue in patients receiving maintenance dialysis: a review of definitions, measures, and contributing factors. American journal of kidney diseases : the official journal of the National Kidney Foundation. 2008;52(2):353-365.

13. Artom M, Moss-Morris R, Caskey F, Chilcot J. Fatigue in advanced kidney disease. Kidney international. 2014;86(3):497-505.

14. Palmer S, Vecchio M, Craig JC, et al. Prevalence of depression in chronic kidney disease: systematic review and meta-analysis of observational studies. Kidney international. 2013;84(1):179-191.

15. Eyre H, Baune BT. Neuroimmunological effects of physical exercise in depression. Brain Behav Immun. 2012;26(2):251-266.

16. Pai MF, Hsu SP, Yang SY, Ho TI, Lai CF, Peng YS. Sleep disturbance in chronic hemodialysis patients: the impact of depression and anemia. Renal failure. 2007;29(6):673-677.

17. Teles F, Amorim de Albuquerque AL, Freitas Guedes Lins IK, Carvalho Medrado P, Falcao Pedrosa Costa A. Quality of life and depression in haemodialysis patients. Psychology, health \& medicine. 2018;23(9):1069-1078. 
18. Tentori F, Elder SJ, Thumma J, et al. Physical exercise among participants in the Dialysis Outcomes and Practice Patterns Study (DOPPS): correlates and associated outcomes. Nephrology, dialysis, transplantation : official publication of the European Dialysis and Transplant Association - European Renal Association. 2010;25(9):3050-3062.

19. Stack AG, Molony DA, Rives T, Tyson J, Murthy BV. Association of physical activity with mortality in the US dialysis population. American journal of kidney diseases : the official journal of the National Kidney Foundation. 2005;45(4):690-701.

20. O'Sullivan D, McCarthy G. An exploration of the relationship between fatigue and physical functioning in patients with end stage renal disease receiving haemodialysis. J Clin Nurs. 2007;16(11c):276-284.

21. Prince SA, Adamo KB, Hamel ME, Hardt J, Connor Gorber S, Tremblay M. A comparison of direct versus self-report measures for assessing physical activity in adults: a systematic review. The international journal of behavioral nutrition and physical activity. 2008;5:56.

22. Andre D PR, Farringdon J, Safier S, Talbott W, Stone R et al. The development of the SenseWear ArmBand, a revolutionary energy assessment device to assess physical activity and lifestyle. 2006; http://www.bodymedia.com/Professionals/Whitepapers/.

23. Hill K, Dolmage TE, Woon L, Goldstein R, Brooks D. Measurement properties of the SenseWear armband in adults with chronic obstructive pulmonary disease. Thorax. 2010;65(6):486-491.

24. Cole PJ, LeMura LM, Klinger TA, Strohecker K, McConnell TR. Measuring energy expenditure in cardiac patients using the Body Media Armband versus indirect calorimetry. A validation study. The Journal of sports medicine and physical fitness. 2004;44(3):262-271.

25. Zamojska S, Szklarek M, Niewodniczy M, Nowicki M. Correlates of habitual physical activity in chronic haemodialysis patients. Nephrology, dialysis, transplantation : official publication of the European Dialysis and Transplant Association - European Renal Association. 2006;21(5):1323-1327.

26. Sheshadri A, Kittiskulnam P, Johansen KL. Higher Physical Activity Is Associated With Less Fatigue and Insomnia Among Patients on Hemodialysis. Kidney international reports. 2019;4(2):285-292.

27. Baria F, Kamimura MA, Avesani CM, et al. Activity-related energy expenditure of patients undergoing hemodialysis. Journal of renal nutrition : the official journal of the Council on Renal Nutrition of the National Kidney Foundation. 2011;21(3):226-234.

28. Avesani $\mathrm{CM}$, Trolonge $\mathrm{S}$, Deléaval $\mathrm{P}$, et al. Physical activity and energy expenditure in haemodialysis patients: an international survey. Nephrology, dialysis, transplantation : official publication of the European Dialysis and Transplant Association - European Renal Association. 2012;27(6):2430-2434.

29. Majchrzak KM, Pupim LB, Chen K, et al. Physical activity patterns in chronic hemodialysis patients: comparison of dialysis and nondialysis days. Journal of renal nutrition : the official journal of the Council on Renal Nutrition of the National Kidney Foundation. 2005;15(2):217-224.

30. Kopple JD, Kim JC, Shapiro BB, et al. Factors affecting daily physical activity and physical performance in maintenance dialysis patients. Journal of renal nutrition : the official journal of the Council on Renal Nutrition of the National Kidney Foundation. 2015;25(2):217-222.

31. Garcia RSA, Lucinda LMF, Ramos FA, et al. Factors Associated With Functional Capacity in Hemodialysis Patients. Artificial organs. 2017;41(12):1121-1126.

32. Gomes Neto M, de Lacerda FFR, Lopes AA, Martinez BP, Saquetto MB. Intradialytic exercise training modalities on physical functioning and health-related quality of life in patients undergoing maintenance hemodialysis: systematic review and meta-analysis. Clinical rehabilitation. 2018;32(9):1189-1202.

33. Junqué Jiménez A, Esteve Simó V, Andreu Periz L, Segura Ortí E. The Relationship between Physical Activity Levels and Functional Capacity in Patients with Advanced Chronic Kidney Disease. Clinical nursing research. 2020:1054773820907757.

34. Manns PJ, Haennel RG. SenseWear Armband and Stroke: Validity of Energy Expenditure and Step Count Measurement during Walking. Stroke research and treatment. 2012;2012:247165. 
35. Craven JL, Rodin GM, Littlefield C. The Beck Depression Inventory as a screening device for major depression in renal dialysis patients. International journal of psychiatry in medicine. 1988;18(4): 365-374.

36. Krupp LB, LaRocca NG, Muir-Nash J, Steinberg AD. The fatigue severity scale. Application to patients with multiple sclerosis and systemic lupus erythematosus. Archives of neurology. 1989;46(10): 1121-1123.

37. Ju A, Unruh ML, Davison SN, et al. Patient-Reported Outcome Measures for Fatigue in Patients on Hemodialysis: A Systematic Review. American journal of kidney diseases : the official journal of the National Kidney Foundation. 2018;71(3):327-343.

38. Tudor-Locke C. BD. How Many Steps Day Are Enough? Preliminary Pedometer Indices for Public Health>. Sports medicine (Auckland, N.Z.). 2004;34:1-8.

39. Bravata D S-SC, Sundaram V, Gienger A, Lin N, Lewis R et al,. Using Pedometers to Increase Physical Activity and Improve Health JAMA - Journal of the American Medical Association. 2007;298(19): 2296-2304.

40. Mercer K, Giangregorio L, Schneider E, Chilana P, Li M, Grindrod K. Acceptance of Commercially Available Wearable Activity Trackers Among Adults Aged Over 50 and With Chronic Illness: A MixedMethods Evaluation. JMIR mHealth and uHealth. 2016;4(1):e7.

41. Mercer K, Li M, Giangregorio L, Burns C, Grindrod K. Behavior Change Techniques Present in Wearable Activity Trackers: A Critical Analysis. JMIR mHealth and uHealth. 2016;4(2):e40.

42. Wieringa FP, Broers NJH, Kooman JP, Van Der Sande FM, Van Hoof C. Wearable sensors: can they benefit patients with chronic kidney disease? Expert review of medical devices. 2017;14(7):505-519.

43. Brys $A D H$, Lenaert B, Van Heugten CM, Gambaro G, Bossola M. Exploring the Diurnal Course of Fatigue in Patients on Hemodialysis Treatment and Its Relation With Depressive Symptoms and Classical Conditioning. J Pain Symptom Manage. 2019;57(5):890-898.e894.

44. Bossola M, Luciani G, Tazza L. Fatigue and its correlates in chronic hemodialysis patients. Blood purification. 2009;28(3):245-252. 

Part ||

The added value of the Experience Sampling Methodology in assessing fatigue in chronic haemodialysis patients 



\section{Chapter 6}

mHealth based Experience Sampling Method to identify fatigue in the context of daily life in haemodialysis patients

Brys ADH, Stifft F, van Heugten CM, Bossola M, Gambaro G, Lenaert B Published in Clin Kidney J. 2021;14(1):245-54 


\section{Abstract}

\section{Objective}

Fatigue in haemodialysis (HD) patients is a prevalent but complex symptom impacted by biological, behavioural, psychological and social variables. Conventional retrospective fatigue questionnaires cannot provide detailed insight in symptom variability in daily life and related factors. The Experience Sampling Methodology (ESM) overcomes these limitations through repeated momentary assessments in patients' natural environments using digital questionnaires. This study aimed to gain in-depth understanding of HD patients' diurnal fatigue patterns and related variables using a mobile Health (mHealth) ESM application, and sought to better understand the nature of their interrelationships.

\section{Methods}

Forty HD patients used the mHealth ESM application for seven days to assess momentary fatigue and potentially related variables, including daily activities, selfreported physical activity, social company, location and mood.

\section{Results}

Multilevel regression analyses of momentary observations ( $N=1777)$ revealed that fatigue varied between and within individuals. Fatigue was significantly related to HD treatment days, type of daily activity, mood and sleep quality. Time-lagged analyses showed that HD predicted higher fatigue scores at a later time point, $\beta=0.22, p=0.013$. Interestingly, higher momentary fatigue also significantly predicted more depressed feelings at a later time point, $\beta=0.05, p=0.019$, but not the other way around.

\section{Conclusion}

ESM offers novel insights in fatigue in chronic HD patients by capturing informative symptom variability in the flow of daily life. Electronic ESM as a clinical application may help to better understand fatigue in HD patients by providing personalized information about its course and relationship with other variables in daily life, paving the way towards personalized interventions. 


\section{Introduction}

Fatigue is one of the most prioritized outcomes among haemodialysis (HD) patients with great impact on their health-related quality of life. ${ }^{1,2}$ However, evidence-based interventions to improve fatigue in HD patients are lacking. This may be attributed to remaining gaps in literature about its underlying aetiology and the lack of valid and reliable measuring methods to assess fatigue, hence the broad prevalence range of $40-80 \% .^{3-5}$ A recently proposed biopsychosocial model to explain fatigue in HD patients posits that fatigue may initially be triggered by chemical imbalances, but may eventually be perpetuated by other factors such as psychological (e.g. depression) and social factors (e.g. poor social support). ${ }^{6}$ Hence, detailed insight in how behavioural, psychological and social variables may affect the course and severity of fatigue in HD patients is needed prior to the development of effective treatments.

Conventionally used measurement instruments for fatigue, such as the Fatigue Severity Scale (FSS) or the SF-36 Vitality Subscale, may hamper an in-depth understanding of this symptom and the factors involved in its development. First, these instruments are often administered at one arbitrary moment in time and evaluate fatigue in general or retrospectively over a preceding period. As such, they rely on memory-based responses which may not provide reliable information about actual symptom experience. ${ }^{7}$ Second, these conventional questionnaires only provide a general picture of fatigue, for instance as an average fatigue severity score over a certain period. However, fatigue symptoms can vary across months, weeks, days, and even within days ${ }^{8,9}$ and this variability may be causally related to diurnal variations in mood, daily activities or social context. ${ }^{10}$ Conventional retrospective questionnaires do not allow assessing this symptom variability and related factors. ${ }^{11}$

The Experience Sampling Method (ESM) is a measuring method that overcomes these limitations. ESM is a structured nowadays digital diary technique that allows investigating symptoms in daily life through their repeated real-time ('here-and-now') assessment as well as potential contributing factors, including behavioural, psychological, and social variables. ${ }^{11}$ Importantly, ESM assesses symptoms in patients' natural environments, which allows identifying informative variability in fatigue and factors related to that variability. Furthermore, repeated measurements provide insight in factors that may prospectively predict improvement or worsening of fatigue, 
paving the way towards personalized interventions targeting these factors. Finally, when incorporated into a mobile Health (mHealth) application, it can be flexibly integrated in the flow of daily life and may therefore reduce non-compliance. ${ }^{12}$ To date, a limited number of studies have used ESM to identify fatigue in HD

patients. ${ }^{8,9,13,14}$ However, they mainly queried fatigue without evaluating contextual variables related to fatigue and without the added benefits of using an mHealth application, such as random assessments throughout the day rather than at fixed time points. This limits adaptation of daily life routines by participants in order to be more available for the questionnaires, thereby threatening reliable data collection (i.e. responsivity). ${ }^{15}$

Therefore, we designed a study to gain in-depth insight in the diurnal variability of fatigue in HD patients using an mHealth ESM application. Second, we investigated whether momentary fatigue was related to variables in daily life including current daily activity, location, social company, mood and self-reported physical activity. Finally, by investigating the temporal dynamics between fatigue and these variables, we sought to better understand the nature of their interrelationship.

\section{Subjects and methods}

\section{Study design and sample}

We conducted a prospective observational study. Participants were recruited at the HD units of Zuyderland Medical Centre in Sittard-Geleen and Heerlen, The Netherlands, between July and August 2019. All prevalent patients on a chronic treatment for at least 6 months were screened for eligibility. Exclusion criteria were: < 18 years of age, insufficient understanding of the Dutch language compromising participation in the study based on clinical judgement, inability to handle the mHealth application independently because of hearing problems, vision problems or insufficient (cognitive) skills based on clinical judgement by the attending physician, diagnosed dementia, chronic fatigue syndrome, fibromyalgia or actual instability of clinical condition requiring hospitalization. The protocol was approved by the local medical ethical committee (METCZ20190078) and registered as NCT04049773 (clinicaltrials.gov). Written informed consent was obtained from all patients prior to participation. 


\section{Data collection}

PsyMate PM $^{T M}$

PsyMate $^{\mathrm{TM}}$ (smartHealth $\mathrm{GmbH}$, Luxembourg) is a smartphone-based mHealth application developed by Maastricht University and Maastricht UMC+ (www.psymate.eu) for momentary assessment of daily life experiences. It was specifically developed to implement ESM in clinical practice. Its user-friendly touchscreen interface makes it accessible for participants with limited technological experience. $^{16}$

The application was programmed to emit ten auditory signals or 'beeps' throughout the day during seven consecutive days at random moments in time between 6.30 AM and 10.30 PM. Beeps were separated by at least 15 minutes. Each beep was a prompt to complete a short self-report questionnaire of 25 items within the application, including statements about momentary fatigue, mood, activity, location and company. Completion of a questionnaire was possible until 15 minutes after the beep. Thereafter, it was closed and registered as missing data. Completion of each ESM questionnaire took 1-2 minutes.

First, participants were requested to respond to the general statement 'I feel tired' on a 7-point Likert scale, ranging from one ('not at all') to seven ('very much'). Whenever participants responded two or higher, two additional statements were presented: 'I feel mentally tired' and 'I feel physically tired'. Next, questions about current activities (e.g. type of activity, self-reported physical or mental activity), mood and context (location, company) were presented. Questions with respect to mood, physical and mental activity were again answered on a 7-point Likert scale, whereas questions about location, company and type of activity were answered in a multiplechoice format (Figure 6.1 and supplemental Table 6.1). The same questions were repeated at every beep and were based on a preset questionnaire, the validity of which has been demonstrated in previous studies using the PsyMate application in several clinical populations ${ }^{17-19}$.

Furthermore, the PsyMate application included a short self-report morning questionnaire containing statements about sleep quality during the previous night as 
well as an end-of-day questionnaire about retrospective evaluation of fatigue and mood during the preceding day (Supplemental Table S6.2).
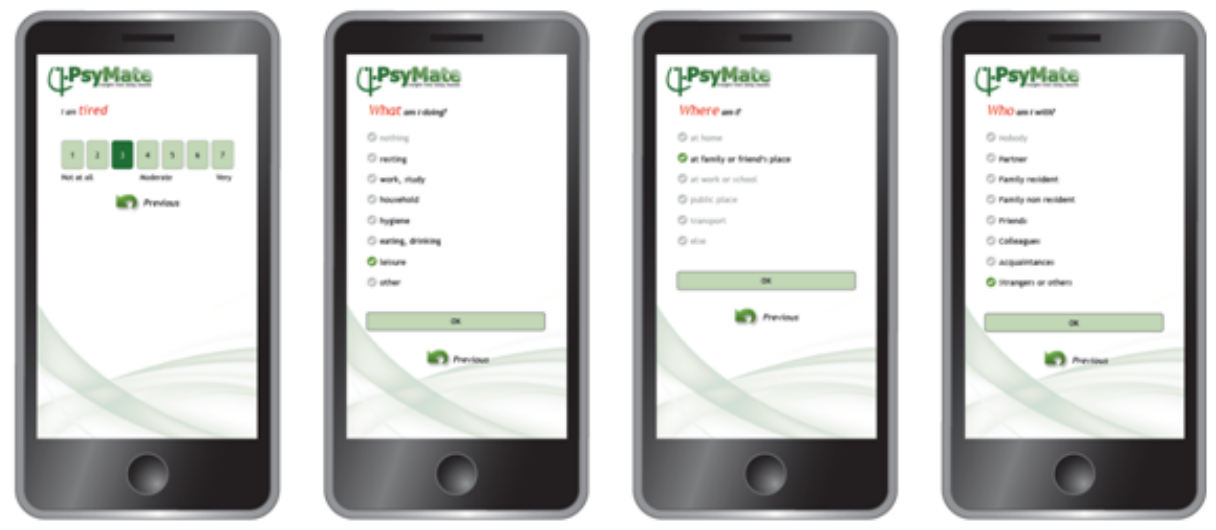

Figure 6.1 PsyMate interface representation of fatigue ESM questionnaire.

\section{Baseline data and laboratory measurements}

Demographical, clinical and laboratory information was obtained from computerized medical records at the hospital. The FSS and Hospital Anxiety and Depression Scale (HADS) were administered to assess average fatigue severity and depressive and anxiety symptoms in the sample. Blood results from routine monthly analysis pre- and postdialysis included complete blood count, iron status, urea, creatinine, electrolytes, serum albumin, Parathyroid Hormone, C-Reactive Protein. Dialysis adequacy was calculated from urea clearance ( $K t / V$ urea clearance).

\section{Haemodialysis treatment}

Routine haemodialysis was performed according to the prescription of the clinic's nephrologist. Either low flux (Gambro Polyflux 17L, Fresenius F6HPS) or high flux (Gambro Polyflux G210H, Fresenius FX100) dialysis membranes were used with a bicarbonate dialysate. The dialysis technique was conventional HD, except for four patients that were on hemodiafiltration. Patients in our sample received dialysis thrice weekly either during the day (3-4 hours) or at night (6-7 hours). Arterial blood flow was 300-400 ml/minute during daytime $\mathrm{HD}$ and $150-200 \mathrm{ml} /$ minute during overnight dialysis. Dialysis rate flow was $400-600 \mathrm{ml} /$ minute for day treatment and 300 
$\mathrm{ml} /$ minute overnight. The desired ultrafiltration volume (mean $1.55 \pm 0.72 \mathrm{~L}$ ) was determined by the treating nephrologist. Most subjects were under regular treatment with recombinant human erythropoietin, antihypertensive medications and other commonly used drugs such as 1,25-dihydroxyvitamin D, phosphate and potassium binders.

\section{Study procedure}

Patients were screened by a researcher on the inclusion and exclusion criteria and received an information letter if considered eligible. After participants gave their written informed consent, the mHealth application PsyMate ${ }^{\mathrm{TM}}$ was installed on their personal smartphone or, in the absence thereof, a substitute iPod Touch (Apple inc.) was provided. The use of the application was explained in detail in a briefing session that took place during the participants' regular treatment. Subsequently, participants were requested to complete momentary assessments for seven consecutive days. Importantly, participants were instructed to continue their normal daily routines. The FSS and HADS were completed on the last study day during regular HD, though one participant did not hand them in. Demographical and clinical information was obtained at the moment of inclusion. Blood results of monthly routine investigation were obtained closest to the last study day.

\section{Data and statistical analysis}

Statistical analysis was performed using SPSS software, version 25. Data are reported as mean and standard deviation (SD) or median and [range] as appropriate. ESM data have a multilevel structure, with observations (level 1 ) nested within individuals (level 2). The intraclass correlation coefficient (ICC) was calculated to assess fatigue variability between and within individuals. In this study, the ICC describes how strongly different observations from the same patient resemble each other. In the context of fatigue for instance, the higher the ICC, the more fatigue should be considered as a trait (i.e. low variability between observations from the same individual) rather than a state (i.e. high variability between observations). Multilevel regression analyses were conducted to investigate the relationship between momentary fatigue and variables of interest. Analyses were run in a random intercept and random slope model, except for the analyses with categorical variables, which employed a random intercept model. All Likert scales ranging from 1 to 7 were 
transformed to 0 to 6 scales in order to be able to meaningfully interpret the intercept. Categorical variables, i.e. type of activity, location and social company were recoded into dummy variables. Reference categories for type of activity, location and company were 'relaxing', 'at home' and 'no one', respectively. To investigate whether type of daily activity, social company and location at a previous time point could predict currently experienced fatigue, time-lagged analyses were used while controlling for fatigue at those previous time points. To investigate the nature of the interrelationship between fatigue and depressed mood, two additional time-lagged analyses were run. In line with previous research, time-lagged analyses were carried out up until 2 previous time points ( $\mathrm{t}-1$ and $\mathrm{t}-2$ ). ${ }^{20}$ The richness of data obtained through ESM allows reliable statistical estimation in relatively small samples of participants (level 2) due to multiple observations within individuals (level 1).

\section{Results}

\section{Sample characteristics}

Forty-two patients participated in this study. Two of them completed less than $30 \%$ of all PsyMate questionnaires and were excluded from the analyses conform ESM guidelines. $^{21}$ The remaining forty patients (31 males) had a mean age of 64.35 (SD=13.69). Their demographical and clinical characteristics are reported in Table 6.1. Participants completed 1777 ESM PsyMate questionnaires, 258 morning and 260 endof-day ESM PsyMate questionnaires, averaging to 57 out of 84 questionnaires per participant ( $68 \%$ compliance rate and $97 \%$ completion rate).

\section{Momentary fatigue}

At the level of the ESM questionnaires, average momentary fatigue was 2.36 $(S D=1.97)$ on a scale from 0 to 6 . Participants responded one or higher to the general statement 'I feel tired' in 1209 completed ESM questionnaires, which means that participants indicated to experience at least some fatigue in $68 \%$ of all random momentary observations. Average physical fatigue was $3.26(S D=1.61)$ and average mental fatigue was 1.71 (SD=1.60). Concurrent daily activity patterns, location and social company patterns are shown in Figure 6.2. Average quality of sleep was 3.92 $(\mathrm{SD}=1.83)$. 
Table 6.1 Participants' baseline characteristics ( $N=40)$.

\begin{tabular}{|c|c|}
\hline Characteristics & Mean \pm SD [range] / n (percentage) \\
\hline \multicolumn{2}{|l|}{ Demographical \& clinical characteristics } \\
\hline Age (years) & $64.4 \pm 13.7[34-84]$ \\
\hline Sex (Male) & $31(77.5 \%)$ \\
\hline $\mathrm{BMI}\left(\mathrm{Kg} / \mathrm{m}^{2}\right)$ & $27.9 \pm 5.9$ \\
\hline Charlson Comorbidity Index & $2.7 \pm 1.6[0-6]$ \\
\hline \multicolumn{2}{|l|}{ Primary kidney disease } \\
\hline Hypertension & $8(20 \%)$ \\
\hline Glomerulonefritis & $2(5 \%)$ \\
\hline Diabetes & $13(32.5 \%)$ \\
\hline Interstitial nefritis & $0(0 \%)$ \\
\hline Polycystic kidney disease & $6(15 \%)$ \\
\hline Other/unknown & $11(27.5 \%)$ \\
\hline Dialysis vintage (months) & $49 \pm 47$ \\
\hline Diuresis/ residual urine output & $14(35 \%)$ \\
\hline Diuresis/ residual urine output $(\mathrm{ml} / 24 \mathrm{~h})^{+}$ & $1044 \pm 687$ \\
\hline Interdialytic weight gain $(\mathrm{Kg})$ & $1.6 \pm 0.7$ \\
\hline UF rate $\mathrm{ml} / \mathrm{h} / \mathrm{Kg}(n=34)$ & $4.35 \pm 2.23$ \\
\hline Anuric patients $(n=26)$ & $4.50 \pm 2.34$ \\
\hline Non-anuric patients $(n=8)$ & $3.87 \pm 1.93$ \\
\hline Day HD treatment $(n=25)$ & $4.82 \pm 2.34$ \\
\hline Night HD treatment $(n=9)$ & $3.06 \pm 1.28$ \\
\hline \multicolumn{2}{|l|}{ Haemodialysis access } \\
\hline Arteriovenous fistula & $30(75 \%)$ \\
\hline Arteriovenous graft & $3(7.5 \%)$ \\
\hline Central venous catheter & $7(17.5 \%)$ \\
\hline \multicolumn{2}{|l|}{ Dialysis group (dialysis hours/treatment) } \\
\hline Morning $(3.5-5.0 \mathrm{~h})$ & $18(45 \%)$ \\
\hline Afternoon $(4.0 \mathrm{~h})$ & $8(20 \%)$ \\
\hline Evening $(4.0 \mathrm{~h})$ & $4(10 \%)$ \\
\hline Night (7-7.25 h) & $10(25 \%)$ \\
\hline \multicolumn{2}{|l|}{ Laboratory characteristics } \\
\hline spKt/V: urea clearance & $1.6 \pm 0.3$ \\
\hline Hemoglobin (mmol/L) & $7.0 \pm 0.6$ \\
\hline Serum albumin $(\mathrm{g} / \mathrm{L})$ & $39.8 \pm 3.4$ \\
\hline Serum creatinine ( $\mu \mathrm{mol} / \mathrm{L})$ & $922.8 \pm 260.8$ \\
\hline Serum urea $(\mathrm{mmol} / \mathrm{L})$ & $23.6 \pm 4.9$ \\
\hline Parathyroid hormone (pmol/L) & $37.8 \pm 26.6$ \\
\hline Calcium (mmol/L) & $2.3 \pm 0.1$ \\
\hline Phosphorus (mmol/L) & $1.7 \pm 0.5$ \\
\hline C-reactive protein $(\mathrm{mg} / \mathrm{L})$ & $9.4 \pm 9.8$ \\
\hline \multicolumn{2}{|l|}{ Conventional questionnaires ( $\mathrm{N}=39)$} \\
\hline Fatigue Severity Scale (FSS) & $5.0 \pm 1.2[2.1-7.0]$ \\
\hline \multicolumn{2}{|l|}{ Hospital Anxiety and Depression Scale (HADS) } \\
\hline Anxiety subscale & $4.5 \pm 3.6[0-14]$ \\
\hline Depression subscale & $4.2 \pm 3.8[0-16]$ \\
\hline
\end{tabular}



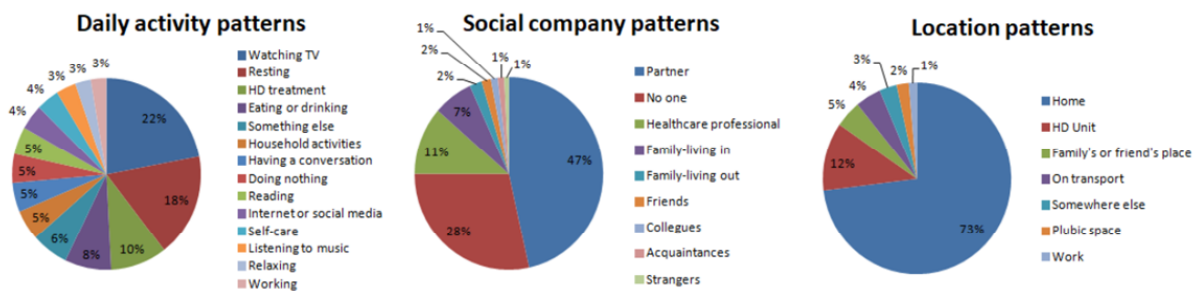

Figure 6.2 Percentages of daily activity $(n=1752)$, social company ( $n=1733)$ and location $(n=1741)$ during momentary assessments.

Figure 6.3 illustrates that fatigue scores differed substantially within individuals and between individuals. The ICC was $0.65,95 \% \mathrm{CI}[0.55,0.75]$, indicating moderate correlation in fatigue observations within individuals.
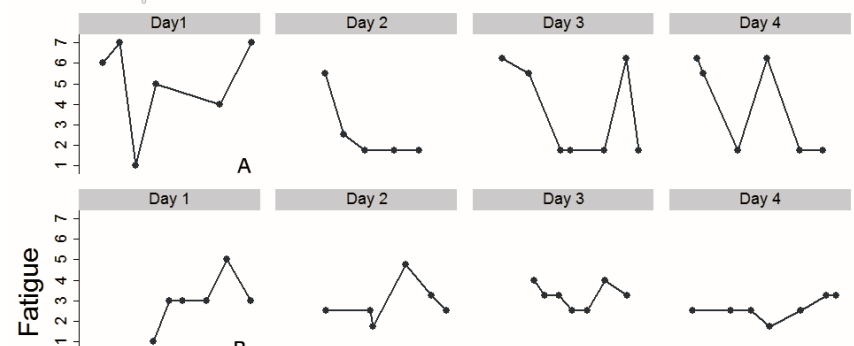

Day 4

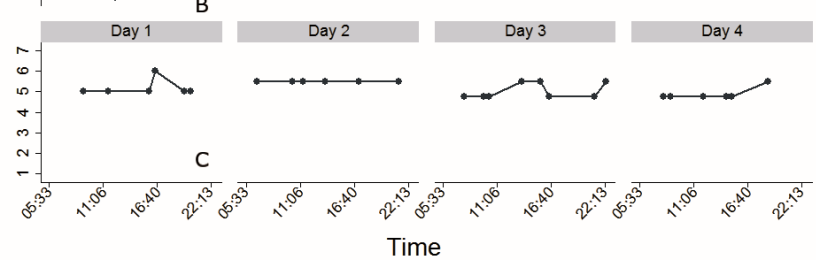

Figure 6.3 Diurnal patterns of momentary fatigue of three different HD patients (subjects $A, B$, and C) during four consecutive study days, illustrating variability in fatigue between as well as within individuals.

Table 6.2 provides a detailed overview of the fixed and random effects of the multilevel models with all of the included predictors of momentary fatigue in HD patients. In these multilevel models, the fixed effects reflect the overall association between a predictor (e.g. physical activity) and fatigue, whereas the random effects reflect individual differences in this association. For these analyses responses to the multiple-choice questions about type of activity, social company and location were clustered together into meaningful categories (see Table 6.2). 
Table 6.2 Overview of multilevel model with momentary fatigue as dependent variable.

\begin{tabular}{|c|c|c|c|c|c|}
\hline \multicolumn{6}{|c|}{ Fixed parameters } \\
\hline Model & Predictor & $\boldsymbol{\beta}$ & SE & $95 \% \mathrm{Cl}$ & $p$-value \\
\hline HD vs. non-HD day & Intercept & 0.33 & 0.06 & {$[0.22,0.44]$} & $<0.001$ \\
\hline Day vs. night treatment & Intercept & 0.47 & 0.58 & {$[-0.71,1.65]$} & 0.426 \\
\hline \multirow[t]{5}{*}{ I Type of daily activity } & Relaxing & 2.42 & 0.25 & {$[1.91,2.93]$} & $<0.001$ \\
\hline & Working & -0.35 & 0.11 & {$[-0.57,-0.13]$} & 0.002 \\
\hline & Internet/talking & -0.33 & 0.10 & {$[-0.53,-0.13]$} & 0.001 \\
\hline & HD treatment/Self care & -0.15 & 0.09 & {$[-0.32,0.02]$} & 0.081 \\
\hline & Eating/drinking & -0.26 & 0.11 & {$[-0.47,-0.05]$} & 0.014 \\
\hline \multirow[t]{6}{*}{$\ddagger$ Social company } & No one & 2.36 & 0.26 & {$[1.84,2.89]$} & $<0.001$ \\
\hline & Family & -0.03 & 0.09 & {$[-0.21,0.15]$} & 0.747 \\
\hline & Friends/acquaintance & -0.15 & 0.19 & {$[-0.52,0.23]$} & 0.444 \\
\hline & Colleagues & -0.50 & 0.28 & {$[-1.05,0.04]$} & 0.071 \\
\hline & Health professionals & -0.11 & 0.12 & {$[-0.32,0.10]$} & 0.292 \\
\hline & Strangers & -0.51 & 0.32 & {$[-1.14,0.12]$} & 0.116 \\
\hline \multirow[t]{5}{*}{ † Location } & At home & 2.37 & 0.25 & {$[1.86,2.88]$} & 0.003 \\
\hline & Family/ friend's place & -0.19 & 0.15 & {$[-0.48,0.10]$} & 0.201 \\
\hline & At work & -0.39 & 0.25 & {$[-0.89,0.11]$} & 0.123 \\
\hline & At the HD unit & -0.10 & 0.09 & {$[-0.27,0.08]$} & 0.294 \\
\hline & Somewhere else & -0.24 & 0.10 & {$[-0.45,-0.04]$} & 0.022 \\
\hline \multirow[t]{2}{*}{ † Positive affect } & Intercept & 4.11 & 0.31 & {$[3.49,4.73]$} & $<0.001$ \\
\hline & Positive affect & -0.40 & 0.05 & {$[-0.50,-0.29]$} & $<0.001$ \\
\hline \multirow[t]{2}{*}{+ Negative affect } & Intercept & 2.16 & 0.24 & {$[1.67,2.64]$} & $<0.001$ \\
\hline & Negative affect & 0.23 & 0.07 & {$[0.08,0.38]$} & 0.004 \\
\hline \multirow[t]{2}{*}{ Physically active } & Intercept & 2.34 & 0.26 & {$[1.82,2.87]$} & $<0.001$ \\
\hline & Physically active & -0.00 & 0.03 & {$[-0.06,0.05]$} & 0.925 \\
\hline \multirow[t]{2}{*}{ Mentally active } & Intercept & 2.27 & 0.26 & {$[1.75,2.79]$} & $<0.001$ \\
\hline & Mentally active & 0.03 & 0.03 & {$[-0.04,0.09]$} & 0.422 \\
\hline \multirow[t]{2}{*}{ Quality of sleep } & Intercept & 2.87 & 0.27 & {$[2.32,3.42]$} & $<0.001$ \\
\hline & Quality of sleep & -0.12 & 0.03 & {$[-0.18,-0.06]$} & $<0.001$ \\
\hline \multicolumn{6}{|c|}{ Random parameters } \\
\hline Model & Predictor & $\beta$ & SE & $95 \% \mathrm{Cl}$ & $p$-value \\
\hline HD vs. non-HD day & Intercept & 2.55 & 0.58 & {$[1.63,3.97]$} & $<0.001$ \\
\hline Day vs. night treatment & Intercept & 2.51 & 0.57 & {$[1.61,3.91]$} & $<0.001$ \\
\hline Type of daily activity & Intercept & 2.53 & 0.57 & {$[1.63,3.95]$} & $<0.001$ \\
\hline Social company & Intercept & 2.54 & 0.58 & {$[1.63,3.97]$} & $<0.001$ \\
\hline Location & Intercept & 2.50 & 0.57 & {$[1.60,3.90]$} & $<0.001$ \\
\hline \multirow[t]{2}{*}{ Positive affect } & Intercept & 2.51 & 0.70 & {$[1.46,4.34]$} & $<0.001$ \\
\hline & Positive affect & 0.04 & 0.02 & {$[0.02,0.10]$} & 0.026 \\
\hline \multirow[t]{2}{*}{ Negative affect } & Intercept & 2.24 & 0.52 & {$[1.42,3.54]$} & $<0.001$ \\
\hline & Negative affect & 0.05 & 0.04 & {$[0.01,0.25]$} & 0.197 \\
\hline \multirow[t]{2}{*}{ Physically active } & Intercept & 2.53 & 0.59 & {$[1.61,3.99]$} & $<0.001$ \\
\hline & Physically active & 0.12 & 0.01 & {$[0.00,0.03]$} & 0.046 \\
\hline \multirow[t]{2}{*}{ Mentally active } & Intercept & 2.52 & 0.58 & {$[1.61,3.96]$} & $<0.001$ \\
\hline & Mentally active & 0.01 & 0.01 & {$[0.00,0.04]$} & 0.159 \\
\hline \multirow[t]{2}{*}{ Quality of sleep } & Intercept & 2.21 & 0.5 & {$[1.35,3.62]$} & $<0.001$ \\
\hline & Quality of sleep & 0.01 & 0.01 & {$[0.00,0.03]$} & 0.117 \\
\hline
\end{tabular}

Note: When the independent variable was dichotomous (i.e. haemodialysis (HD) day versus non-HD day, and day versus night dialysis treatment) or nominal (i.e. type of activity, social company and location) the 
intercept refers to the value of momentary fatigue (on a 7-point Likert scale from 0 to 6 ) when considering the reference situation (i.e. HD day, dialysis day treatment, relaxing, no one's company and at home, respectively). With respect to all the other variables (i.e. positive and negative affect, extent of mental or physical activity, and sleep quality) the intercept refers to the value of momentary fatigue when the independent variable was zero (on a 7-point Likert scale from 0 (e.g. meaning "not at all" feeling cheerful, down, or slept well) to 6 (e.g. meaning "very much"). \$ With respect to type of activity, 'doing nothing', 'resting', 'relaxing', 'listening to music', 'watching television', and 'reading' were combined in the (reference) category 'relaxing'. 'Work' and 'household activities' were clustered as 'working'. 'Undergoing HD Treatment' and 'self-care' were also clustered. 'Talking', 'internet' and 'social media' were put together as well. Social company selection options being 'with one's partner' , 'with a family member living-in or outliving' were combined into one single 'family' item. 'Friends and acquaintances' were put together. Location options of 'being in a public place', 'on transportation' or 'somewhere else' were also combined. † PsyMate items with respect to a positive state of mind (i.e. 'feeling cheerful', 'relaxed, 'satisfied' and 'confident') were combined to a single variable of 'positive affect' for these analyses. 'Feeling anxious', 'down' ,'agitated' and 'powerless' were put together in a 'negative affect' variable.

Fatigue scores were significantly higher on HD treatment days compared to non-HD treatment days. Fatigue ratings did not differ significantly between patients on day or night HD treatment. With respect to type of daily activity, all activities were judged as significantly less fatiguing than relaxing (reference category), except for receiving HD treatment, which did not differ significantly from the reference category. With respect to social company or location, no significant differences in fatigue were found between the respective categories, except for the category 'somewhere else' (public place, transport, somewhere else) which was significantly less fatiguing than being at home (reference category). With respect to physical or mental activity, we found no significant associations with fatigue measured at the same moment in time. In contrast, participants' mood was significantly correlated with momentary fatigue scores, with negative affect related to higher fatigue and positive affect to lower momentary fatigue ratings. Finally, all fatigue scores during the day were significantly lower if the quality of sleep during the previous night was higher.

\section{Temporal relationship}

HD treatment at previous time points ( $t-1$ and $t-2$ ) predicted significantly higher current fatigue scores relative to the reference category (relaxing), while controlling for fatigue at those previous time points, $\beta=0.18, S E=0.08, p=0.030,95 \% \mathrm{Cl}[0.17,0.35]$ for $\mathrm{t}-1$ and $\beta=0.22, \mathrm{SE}=0.09, p=0.013,95 \% \mathrm{Cl}[0.05,0.39]$ for $\mathrm{t}-2$. Subsequent analyses showed that this temporal relationship only applied to anuric patients $(n=26)(\beta=0.23$, $S E=0.10, p=0.029,95 \% \mathrm{Cl}[0.23,0.43]$ for $\mathrm{t}-1$ and $\beta=0.22, S E=0.11, p=0.042,95 \% \mathrm{Cl}$ $[0.01,0.43]$ for $t-2)$. The ultrafiltration volume and ultrafiltration rate did not 
significantly predict this relationship. Other daily activities measured earlier in time (t-1 and t-2) did not differ significantly relative to the reference category in predicting current fatigue. Being in the company of a health care professional, being with a stranger and being at the HD unit (Supplemental table 1) at a previous time point (t-1) significantly predicted current fatigue ratings relative to the reference categories (i.e. $\beta=0.26, S E=0.10, p=0.015,95 \% \mathrm{Cl}[0.05,0.46]$ for being with a health care professional, $\beta=0.84, S E=0.32, p=0.008,95 \% \mathrm{Cl}[0.22,1.46]$ for being with a stranger, and $\beta=0.35, S E=0.09, p<0.001,95 \% \mathrm{Cl}[0.18,0.53]$ for being at the HD unit). All other social company and location items did not differ significantly from the reference categories in predicting current fatigue (neither on $\mathrm{t}-1$, nor on $\mathrm{t}-2$ ).

In order to investigate the direction of the relationship between fatigue and depressed mood, two time-lagged analyses were run. In the first model, fatigue was the dependent variable and depressed mood earlier in time was the predictor variable (while controlling for fatigue earlier in time). In the second model, depressed mood was the dependent variable and fatigue earlier in time was the predictor variable (while controlling for depressed mood earlier in time). Momentary fatigue did not differ significantly when individuals reported to feel down at previous measured time points ( $t-1$ or $t-2$ ) while controlling for their previously reported momentary fatigue scores. Reversely, momentary scores of depressed mood were significantly higher if participants reported more fatigue at the previous time point $(\mathrm{t}-1)$, controlling for depressed mood at that previous moment ( $\mathrm{t}-1), \beta=0.05, S E=0.02, p=0.019,95 \% \mathrm{Cl}$ $[0.01,0.10]$. No significant relationship was found at t-2.

\section{Discussion}

This is the first study using an mHealth application for Experience Sampling Methodology to investigate fatigue in the flow of daily life in chronic HD patients. Moreover, it is the first study that extensively demonstrated variability in fatigue symptoms between and within HD patients and revealed several behavioural, social and psychological factors that were associated with momentary fatigue.

First, participants reported significantly less fatigue during daily activities such as work, household activities, eating, drinking, using the internet or social media or 
having a conversation than when they reported to be relaxing. Time-lagged analysis further showed that relaxing did not predict significantly higher fatigue levels at a later moment in time relative to other daily activities. Together, these results suggest that relaxing should be considered as a behavioural response to fatigue that is already present, rather than fatigue worsening as a consequence of prolonged resting. These findings are in line with previous studies, based on qualitative in-depth interviews, investigating how fatigue in HD patients relates to activities in daily life. ${ }^{10,22}$ In these interviews HD patients retrospectively stated to adjust their activities depending on their fatigue levels. Moreover, it may be possible that participants were more fatigueaware when they were not distracted by other activities. Interestingly, results also showed that undergoing HD was not significantly different from relaxing in terms of experienced fatigue. In addition, time-lagged analyses revealed that receiving HD predicted significantly higher fatigue levels at a later time point relative to the reference category. This is further corroborated by the finding that subjects reported more fatigue on HD treatment days relative to non-treatment days. These findings are in line with a previous study demonstrating fatigue increases significantly after HD treatment. ${ }^{8}$ Moreover, it supports the concept of different fatigue patterns in HD patients (i.e. more general fatigue versus fatigue as a response to treatment) ${ }^{14}$ and may suggest they should, at least partially, be distinguished in HD patients.

Furthermore, feelings of negative affect, including depressive mood, were associated with significantly higher momentary fatigue ratings. Extensive literature exists about the association between fatigue and low mood in HD patients. ${ }^{6,8,23-25}$ However, due to cross-sectional study designs the nature of their interrelationship remains unclear. In this study, time-lagged analysis revealed higher levels of depressive mood after feeling fatigued at an earlier point in time. The reverse relationship was not found, suggesting that low mood may be secondary to fatigue in HD patients. However, the small effect sizes indicate that other factors should be taken into consideration when trying to explain the relationship between fatigue and depressive symptoms (e.g. daily activity or physical activity).

Finally, the most important finding from a clinical point of view may be the extent of individual differences in fatigue between and within subjects as well as the factors related to fatigue. Therefore, potential effectiveness of a one-size-fits-all treatment for fatigue seems limited, a priori. A more personalized approach in treatment, 
instead, may be more beneficial. The advantage of ESM is precisely to obtain detailed and personalized insights in the relationship between symptoms and environmental variables enabling interventions to be tailored to the needs of the individual.

A patient-reported outcome measure for fatigue incorporated in an mHealth ESM may have the potential to become a valid and reliable measurement instrument to assess fatigue in the natural environment of HD patients. This resonates with the need for valid and reliable patient-reported outcome measures (PROMs) for fatigue in HD patients expressed in 2016 by the SONG HD fatigue working group. ${ }^{26}$ Moreover, ESM may provide concrete entry points to develop and monitor personalized interventions to alleviate fatigue and improve HD patients' quality of life.

Major strengths of this study include using ESM measurements in daily life to obtain ecologically valid data about fatigue in HD patients, the use of time-lagged analyses to unveil temporal dynamics between symptoms, and the inclusion of both patients receiving HD during daytime and patients being treated overnight. Limitations include a potential selection bias due to a certain level of cognitive capacities needed to use the mHealth application. Further, males were overrepresented in our study, notwithstanding a gender imbalance of $60 \%$ men receiving HD in European countries, including the Netherlands. ${ }^{27}$

In conclusion, fatigue in HD patients is a prevalent but complex phenomenon that differs greatly between and within individuals over time. ESM measurement instruments allow for capturing informative symptom variability and enable linking this to patterns of daily life. By providing more detailed and personalized insight in fatigue symptoms and related factors, ESM paves the way towards personalized intervention. Furthermore, our results provide real-life evidence about the temporal dynamics of the interrelationship between fatigue and depressive symptoms. In our view, an important next step is to develop and implement a reliable and valid PROM for fatigue in HD patients in an electronic ESM measurement instrument. Moreover, investigating the effect of interdisciplinary interventions to improve HD patients' mood on their fatigue experience may be an interesting goal for further research. 


\section{Supplemental material}

Table S6.1 ESM PsyMate: questions.

\begin{tabular}{|c|c|c|}
\hline Domain & Item & Scale type \\
\hline \multirow[t]{9}{*}{ Mood } & 1. I feel cheerful & 7-point Likert scale (1 "not at all" to 7 "very much") \\
\hline & 2. I feel relaxed & 7-point Likert scale (1 "not at all” to 7 "very much") \\
\hline & 3. I feel satisfied & 7-point Likert scale (1 "not at all" to 7 "very much") \\
\hline & 4. I feel anxious & 7-point Likert scale (1 "not at all" to 7 "very much") \\
\hline & 5. I feel down & 7-point Likert scale ( 1 "not at all" to 7 "very much") \\
\hline & 6. I feel confident & 7-point Likert scale (1 "not at all" to 7 "very much") \\
\hline & 7. I feel irritable & 7-point Likert scale (1 "not at all" to 7 "very much") \\
\hline & 8. I feel powerless & 7-point Likert scale ( 1 "not at all" to 7 "very much") \\
\hline & 9. I am worrying & 7-point Likert scale (1 "not at all" to 7 "very much") \\
\hline \multirow{7}{*}{$\begin{array}{l}\text { Context } \\
\text { activity }\end{array}$} & 10. What am I doing & Doing nothing, resting, undergoing HD treatment, watching \\
\hline & (just before the beep)? & $\begin{array}{l}\text { TV, reading, listening to music, internet or social media, work, } \\
\text { household, self care, eating or drinking, having a } \\
\text { conversation, relaxing, something else }\end{array}$ \\
\hline & 11. And also? & $\begin{array}{l}\text { resting, undergoing HD treatment, watching TV, reading, } \\
\text { listening to music, internet or social media, work, household, } \\
\text { self care, eating or drinking, having a conversation, relaxing, } \\
\text { something else }\end{array}$ \\
\hline & 12. And...? & $\begin{array}{l}\text { resting, undergoing HD treatment, watching TV, reading, } \\
\text { listening to music, internet or social media, work, household, } \\
\text { self care, eating or drinking, having a conversation, relaxing; } \\
\text { something else }\end{array}$ \\
\hline & 13. I can do this well & 7-point Likert scale (1 "not at all" to 7 "very much") \\
\hline & 14. This is difficult for me & 7-point Likert scale (1 "not at all" to 7 "very much") \\
\hline & 15. I would rather be doing something else & 7-point Likert scale (1 "not at all" to 7 "very much") \\
\hline $\begin{array}{l}\text { Context } \\
\text { location }\end{array}$ & 16. Where am I? & $\begin{array}{l}\text { At the HD unit, at home, at family's/friend's place, at work, } \\
\text { public space, transport, somewhere else }\end{array}$ \\
\hline \multirow[t]{3}{*}{$\begin{array}{l}\text { Context } \\
\text { social }\end{array}$} & 17. Who am I with? & $\begin{array}{l}\text { No one, healthcare professional, partner, family living in, } \\
\text { family out-living, friends, colleagues, acquaintances, } \\
\text { strangers }\end{array}$ \\
\hline & 18. And also? & $\begin{array}{l}\text { healthcare professional, partner, family living in, family out- } \\
\text { living, friends, colleagues, acquaintances, strangers }\end{array}$ \\
\hline & 19. And...? & $\begin{array}{l}\text { healthcare professional, partner, family living in, family out- } \\
\text { living, friends, colleagues, acquaintances, strangers }\end{array}$ \\
\hline \multirow[t]{4}{*}{ Fatigue } & 20. I feel tired & 7-point Likert scale (1 "not at all" to 7 "very much") \\
\hline & Branched questions when feeling tired $>1$ & \\
\hline & 20a. I feel mentally tired & 7-point Likert scale (1 "not at all" to 7 "very much") \\
\hline & 20b. I feel physically tired & 7-point Likert scale (1 "not at all" to 7 "very much") \\
\hline \multirow[t]{7}{*}{ Physical } & 21. I am suffering from a headache & 7-point Likert scale ( 1 "not at all" to 7 "very much") \\
\hline & 22. I am suffering from muscle cramps & 7-point Likert scale ( 1 "not at all" to 7 "very much") \\
\hline & 23. I am suffering from pain in bones or joints & 7-point Likert scale (1 "not at all" to 7 "very much") \\
\hline & 24. I am suffering from itch & 7-point Likert scale ( 1 "not at all" to 7 "very much") \\
\hline & 25. I am suffering from restless legs & 7-point Likert scale (1 "not at all" to 7 "very much") \\
\hline & 26. I have been physically active before the beep & 7-point Likert scale ( 1 "not at all" to 7 "very much") \\
\hline & 27. I have been mentally active before the beep & 7-point Likert scale ( 1 "not at all" to 7 "very much") \\
\hline General & 28. This beep disturbed me & 7-point Likert scale ( 1 "not at all" to 7 "very much") \\
\hline
\end{tabular}


Table S6.2 ESM PsyMate questionnaires.

Morning questionnaire

\begin{tabular}{|c|c|c|}
\hline Domain & Item & Scale type \\
\hline $\begin{array}{l}\text { Sleep } \\
\text { and night } \\
\text { rest }\end{array}$ & $\begin{array}{l}\text { 1. I slept well } \\
2 \text {. How long did it take before I felt asleep } \\
\text { yesterday evening } \\
\text { 3. How often did I wake up last night } \\
\text { 4. I feel rested }\end{array}$ & $\begin{array}{l}\text { 7-point Likert scale ( } 1 \text { "not at all" to } 7 \text { "very good") } \\
0-5 \mathrm{~min}, 5-15 \mathrm{~min}, 15-30 \mathrm{~min}, 30-45 \mathrm{~min}, 45 \mathrm{~min}- \\
\text { 1hour, } 1-2 \text { hours, } 2-4 \text { hours, }>4 \text { hours } \\
1 \text { time, } 2 \text { times, } 3 \text { times, } 4 \text { times, } 5 \text { times, }>5 \text { times } \\
\text { 7-point Likert scale ( } 1 \text { "not at all" to } 7 \text { "very much") }\end{array}$ \\
\hline Fatigue & $\begin{array}{l}\text { 5. I feel tired } \\
\text { Branched questions when feeling tired }>1 \\
\text { 5a. I feel mentally tired } \\
\text { 5b. I feel physically tired } \\
6 . \text { I do not look forward to this day }\end{array}$ & $\begin{array}{l}\text { 7-point Likert scale ( } 1 \text { "not at all" to } 7 \text { "very much") } \\
\text { 7-point Likert scale ( } 1 \text { "not at all" to } 7 \text { "very much") } \\
\text { 7-point Likert scale ( } 1 \text { "not at all" to } 7 \text { "very much") }\end{array}$ \\
\hline
\end{tabular}

Evening questionnaire

\begin{tabular}{|c|c|c|}
\hline Domain & Item & Scale type \\
\hline General & $\begin{array}{l}\text { 1. Today was an ordinary day } \\
\text { 2. Without the application I would have } \\
\text { done other things today }\end{array}$ & $\begin{array}{l}\text { 7-point Likert scale ( } 1 \text { "not at all" to } 7 \text { "very much") } \\
\text { 7-point Likert scale ( } 1 \text { "not at all" to } 7 \text { "very much") }\end{array}$ \\
\hline Fatigue & $\begin{array}{l}\text { 3. Overall, I felt tired today } \\
\text { 4. Overall, I felt mentally tired today } \\
\text { 5. Overall, I felt physically tired today }\end{array}$ & $\begin{array}{l}\text { 7-point Likert scale ( } 1 \text { "not at all" to } 7 \text { "very much") } \\
7 \text {-point Likert scale ( } 1 \text { "not at all" to } 7 \text { "very much") } \\
7 \text {-point Likert scale ( } 1 \text { "not at all" to } 7 \text { "very much") }\end{array}$ \\
\hline Mood & $\begin{array}{l}\text { 6. Overall, I felt good today } \\
\text { 7. Overall, I felt tense today } \\
\text { 8. Overall, I've been worrying today }\end{array}$ & $\begin{array}{l}\text { 7-point Likert scale ( } 1 \text { "not at all" to } 7 \text { "very much") } \\
\text { 7-point Likert scale ( } 1 \text { "not at all" to } 7 \text { "very much") } \\
\text { 7-point Likert scale ( } 1 \text { "not at all" to } 7 \text { "very much") }\end{array}$ \\
\hline Medication & 9. Today, I took my medication & 7-point Likert scale (1 "nothing" to 7 "everything") \\
\hline
\end{tabular}




\section{References}

1. Davison SN, Jhangri GS. Impact of pain and symptom burden on the health-related quality of life of hemodialysis patients. J Pain Symptom Manage. 2010;39(3):477-485.

2. Urquhart-Secord R, Craig JC, Hemmelgarn B, et al. Patient and Caregiver Priorities for Outcomes in Hemodialysis: An International Nominal Group Technique Study. American journal of kidney diseases : the official journal of the National Kidney Foundation. 2016;68(3):444-454.

3. Jhamb M, Weisbord SD, Steel JL, Unruh M. Fatigue in patients receiving maintenance dialysis: a review of definitions, measures, and contributing factors. American journal of kidney diseases : the official journal of the National Kidney Foundation. 2008;52(2):353-365.

4. Artom M, Moss-Morris R, Caskey F, Chilcot J. Fatigue in advanced kidney disease. Kidney international. 2014;86(3):497-505.

5. Jhamb M, Argyropoulos C, Steel JL, et al. Correlates and outcomes of fatigue among incident dialysis patients. Clinical journal of the American Society of Nephrology : CJASN. 2009;4(11):1779-1786.

6. Picariello F, Moss-Morris R, Macdougall IC, Chilcot AJ. The role of psychological factors in fatigue among end-stage kidney disease patients: a critical review. Clin Kidney J. 2017;10(1):79-88.

7. Van den Bergh O, Walentynowicz M. Accuracy and bias in retrospective symptom reporting. Current opinion in psychiatry. 2016;29(5):302-308.

8. Brys $A D H$, Lenaert B, Van Heugten CM, Gambaro G, Bossola M. Exploring the Diurnal Course of Fatigue in Patients on Hemodialysis Treatment and Its Relation With Depressive Symptoms and Classical Conditioning. J Pain Symptom Manage. 2019;57(5):890-898.e894.

9. Abdel-Kader K, Jhamb M, Mandich LA, et al. Ecological momentary assessment of fatigue, sleepiness, and exhaustion in ESKD. BMC nephrology. 2014;15:29.

10. Picariello F, Moss-Morris R, Macdougall IC, Chilcot J. 'It's when you're not doing too much you feel tired': A qualitative exploration of fatigue in end-stage kidney disease. British journal of health psychology. 2018;23(2):311-333.

11. Shiffman S, Stone AA, Hufford MR. Ecological momentary assessment. Annual review of clinical psychology. 2008;4:1-32.

12. Stone AA, Shiffman S, Schwartz JE, Broderick JE, Hufford MR. Patient compliance with paper and electronic diaries. Controlled clinical trials. 2003;24(2):182-199.

13. Riis J, Loewenstein G, Baron J, Jepson C, Fagerlin A, Ubel PA. Ignorance of hedonic adaptation to hemodialysis: a study using ecological momentary assessment. Journal of experimental psychology. General. 2005;134(1):3-9.

14. Horigan AE, Barroso JV. A Comparison of Temporal Patterns of Fatigue in Patients on Hemodialysis. Nephrology nursing journal : journal of the American Nephrology Nurses' Association. 2016;43(2):129138, 148, quiz 139.

15. Edwards CJ, Cella M, Tarrier N, Wykes T. The optimisation of experience sampling protocols in people with schizophrenia. Psychiatry research. 2016;244:289-293.

16. Myin-Germeys I, Birchwood M, Kwapil T. From environment to therapy in psychosis: a real-world momentary assessment approach. Schizophrenia bulletin. 2011;37(2):244-247.

17. Lenaert B, Colombi M, van Heugten C, Rasquin S, Kasanova Z, Ponds R. Exploring the feasibility and usability of the experience sampling method to examine the daily lives of patients with acquired brain injury. Neuropsychological rehabilitation. 2019;29(5):754-766.

18. Wichers M, Peeters F, Rutten BP, et al. A time-lagged momentary assessment study on daily life physical activity and affect. Health psychology : official journal of the Division of Health Psychology, American Psychological Association. 2012;31(2):135-144. 
19. Oorschot $M$, Lataster $T$, Thewissen V, et al. Emotional experience in negative symptoms of schizophrenia--no evidence for a generalized hedonic deficit. Schizophrenia bulletin. 2013;39(1): 217-225.

20. Lenaert B, Neijmeijer M, van Kampen N, van Heugten C, Ponds R. Poststroke Fatigue and Daily Activity Patterns During Outpatient Rehabilitation: An Experience Sampling Method Study. Archives of physical medicine and rehabilitation. 2020;101(6):1001-1008.

21. Delespaul P.A.E.G. Assessing Schizophrenia in Daily Life: The Experience Sampling Method, Maastricht University, 1995.

22. Horigan AE, Schneider SM, Docherty S, Barroso J. The experience and self-management of fatigue in patients on hemodialysis. Nephrology nursing journal : journal of the American Nephrology Nurses' Association. 2013;40(2):113-122, quiz 123.

23. Bossola M, Luciani G, Tazza L. Fatigue and its correlates in chronic hemodialysis patients. Blood purification. 2009;28(3):245-252.

24. Bossola M, Di Stasio E, Giungi S, Rosa F, Tazza L. Fatigue is associated with serum interleukin-6 levels and symptoms of depression in patients on chronic hemodialysis. I Pain Symptom Manage. 2015;49(3):578-585.

25. Palmer S, Vecchio M, Craig JC, et al. Prevalence of depression in chronic kidney disease: systematic review and meta-analysis of observational studies. Kidney international. 2013;84(1):179-191.

26. Nair D, Finkelstein FO. Toward Developing a Patient-Reported Outcome Measure for Fatigue in Hemodialysis. American journal of kidney diseases : the official journal of the National Kidney Foundation. 2019;74(2):151-154.

27. Kramer A, Pippias M, Noordzij M, et al. The European Renal Association - European Dialysis and Transplant Association (ERA-EDTA) Registry Annual Report 2015: a summary. Clin Kidney J. 2018;11(1):108-122. 



\section{Chapter 7}

\section{Unravelling fatigue in haemodialysis patients: comparing retrospective reports to real-time assessments with an $\mathrm{mHealth}$ Experienced Sampling Method}

Brys ADH, Stifft F, van Heugten CM, Bossola M, Gambaro G, Lenaert B Published in J Pain Symptom Manage. 2020; 60(6):1100-1108.e2 


\section{Abstract}

\section{Context}

Fatigue is prevalent among haemodialysis (HD) patients and associated with depressed mood. To advance our understanding of its aetiology and develop appropriate treatments, reliable measurement instruments are needed. However, conventional fatigue and mood questionnaires are prone to bias due to their retrospective nature and may misrepresent or overestimate actual symptom experience (i.e. the so-called 'memory-experience' gap). Experience Sampling Methodology (ESM) overcomes this limitation through repeated real-time assessments in patients' natural environment, thereby providing reliable and ecologically valid data.

\section{Objectives}

We investigated to what extent retrospective symptom reporting accurately represents real-time experiences of fatigue and mood in HD patients using an ESM mobile Health application (PsyMate ${ }^{\mathrm{TM}}$ ).

\section{Methods}

Forty HD patients used the PsyMate ${ }^{\mathrm{TM}}$ for one week to assess real-time fatigue and mood. Additionally, they retrospectively evaluated their symptom experience completing end-of-day and end-of-week questionnaires; and the conventional Fatigue Severity Scale (FSS) and Hospital Anxiety and Depression Scale (HADS).

\section{Results}

Results of real-time observations $(N=1777)$ showed that fatigue and mood varied between and within individuals. Retrospective end-of-week fatigue evaluation was significantly higher than the average real-time fatigue score, $t(38)=3.54, p=0.001$, $d=0.57$. FSS and HADS correlated moderately to strong with the average ESM score for fatigue and mood: $r=0.66$ and $r=0.77$, respectively.

\section{Conclusion}

Retrospective fatigue assessment may lead to overestimation of real-time symptom experience. ESM provides detailed insight and personalized information about symptom experiences which may be crucial for the design of more targeted and personalized interventions for fatigue and mood problems in HD patients. 


\section{Introduction}

Fatigue, with a prevalence range of $40-80 \%$, is one of the most prioritized outcomes among haemodialysis (HD) patients and greatly impacts their health related quality of life (HRQoL). ${ }^{1,2}$ Among biological, psychological and social variables under investigation, so far only a solid association between fatigue and depressive mood has been demonstrated. ${ }^{3-5}$ Moreover, reliable measurement instruments to assess fatigue in HD patients are lacking. ${ }^{4}$ This further impedes the development of appropriate therapies.

Conventional measurement instruments, such as the commonly used Fatigue Severity Scale (FSS), are limited in their clinical applicability in that they only provide a general picture of fatigue severity without giving detailed insight in diurnal variations and related factors in daily life which may contribute to variability in fatigue. Additionally, their reliability to measure symptom fluctuation over time may be questionable as there is report of a high level of faked compliance due to backfilling of retrospective paper diaries. ${ }^{6}$ Perhaps even more importantly, conventional questionnaires are prone to memory bias due to their retrospective nature and may therefore misrepresent actual symptom experience. For instance, retrospective symptom reports have been shown to overestimate actual symptom experience in various clinical populations (i.e. the so-called 'memory-experience gap'). ${ }^{7}$ Further, symptom recall may be influenced by symptom variability and intensity during recall as well as by emotional states at the time of recall or during symptom experiences. Longer time intervals between symptom experience and recall may also affect accuracy. Accurate recall may be further impacted by cognitive heuristics used by patients, such as the peak-end effect where more importance is given to more intense or more recent experiences. $^{8,9}$

The Experience Sampling Methodology (ESM) resolves these constraints by repeatedly measuring symptoms and potential contributing factors, such as behavioural, psychological and social variables, in-the-moment (i.e. momentary) and in patients' natural environments in the flow of daily life. ${ }^{10}$ In a way, ESM captures the film rather than an instantaneous photograph of patients' symptom experiences in daily life. Therefore, ESM not only allows to identify informative variability in symptoms and factors related to that variability, but also overcomes ecological and recall bias. 
Moreover, digitalized ESM, such as an ESM mobile Health (mHealth) application, using time-stamps may reduce faked compliance and can be easily integrated in patients' daily lives. ${ }^{6}$

Since conventional retrospective questionnaires are often used to inform diagnostic and therapeutic decisions, it is relevant to better understand how retrospective symptom reports correspond to actual day-to-day experiences. Previous studies comparing ESM and retrospective questionnaires for symptoms such as pain, fatigue or dyspnea in somatically ill subjects (e.g. rheumatoid arthritis, stroke, irritable bowel syndrome), psychiatric patients (e.g. schizophrenia) and healthy individuals, have shown that retrospective symptom reports often overestimate the intensity of the experienced symptoms, which supports the abovementioned memory-experience gap. ${ }^{8,9,11-14}$ Moreover, some studies demonstrated a peak-end effect for retrospective pain evaluation, but not (consistently) for fatigue. Further, a number of studies investigated fatigue and its course in HD patients' daily life using an ESM technique. ${ }^{5,15-18}$ They mainly showed that fatigue is dynamic, changes over time and may be determined by mood or contextual variables such as activities, social company or location. However, none of these studies reported a comparison between momentary assessments and conventional retrospective evaluation of fatigue.

Therefore, we designed a study to investigate the relationship between momentary assessments and retrospective reporting of fatigue in HD patients. To this end, we collected real-time symptom reports using an mHealth ESM application during seven consecutive days; as well as retrospective symptom reports over the same period at the end of every day and finally at the end of the week. This allowed us to investigate the time period after which differences (if any) between momentary and retrospective reports occur. Additionally, we collected the FSS and Hospital Anxiety and Depression Scale (HADS) to assess to what extent conventional retrospective evaluation of fatigue and mood correlated to real-time assessments; and whether individuals scoring below or above these conventional measures' cut-off scores also differed in their symptom experience in daily life.

The primary aim of this study was to investigate how retrospective reports and conventional measurement instruments for fatigue and mood correspond to the actual day-to-day symptom experiences. The secondary aim was to explore which 
aspect of momentary fatigue and mood is best captured by retrospective evaluation: the average, the highest level (peak) or the most recent score (end). Lastly, we explored to what extent mood may be related to a memory-experience gap in fatigue experience in HD patients given that previous research showed a positive relationship between negative affect and subjective symptom reporting. ${ }^{19}$

\section{Materials and methods}

\section{Study population and design}

Between July and August 2019, patients receiving HD for at least 6 months at the HD units of Zuyderland Medical Centre in Sittard and Heerlen, the Netherlands, were enrolled in this prospective observational study. Exclusion criteria were: $<18$ years old, insufficient understanding of the Dutch language compromising participation in the study based on clinical judgement, patients unable to handle the mHealth application independently because of hearing problems, vision problems or insufficient (cognitive) skills based on clinical judgement by the attending physician, patients with diagnosed dementia, chronic fatigue syndrome, fibromyalgia or actual instability of their clinical condition requiring hospitalization. All patients gave written informed consent and the study protocol was approved by the institutional review board (medical ethical committee of Zuyderland Medical Centre (METCZ20190078)) and registered NCT04049773 (clinicaltrials.gov).

\section{Symptom assessment}

\section{Experience Sampling Method}

To assess real-time fatigue and mood (e.g. feeling cheerful, relaxed, satisfied or confident to express positive affect and feeling anxious, down, irritable or powerless as an expression of negative affect, respectively) in daily life, a digital ESM was used by means of the PsyMate ${ }^{\mathrm{TM}}$ (smartHealth $\mathrm{GmbH}$, Luxembourg). The PsyMate ${ }^{\mathrm{TM}}$ is a user-friendly smartphone-based mHealth application developed by Maastricht University and Maastricht UMC+ (www.psymate.eu) to implement ESM in clinical practice. $^{20}$ 
During seven consecutive days, the PsyMate ${ }^{\mathrm{TM}}$ gave ten auditory ('beep') signals at random time points throughout the day between 6.30 AM and 10.30 PM. Beeps were separated by at least 15 minutes. At each beep, patients were requested to complete a short self-report questionnaire of 25 items on their smartphone including statements about momentary fatigue and mood, using a 7-point Likert scale ranging from one ('not at all') to seven ('very much') (Supplemental Table S7.1, Figure 7.1). Whenever patients gave a score of two or higher to the general statement 'I feel tired', two additional questions were presented: 'I feel mentally tired' and 'I feel physically tired'. Fifteen minutes after each beep signal, the questionnaire was shut down and registered as missing data if it was not filled in. The administered set of questions was based on a preset questionnaire applied in previous studies, using the PsyMate $^{\mathrm{TM}}$ application in other clinical populations. ${ }^{21-23}$ These studies demonstrated good internal consistency of the items to measure mood and self-reported symptoms. They also demonstrated the PsyMate ${ }^{\mathrm{TM}}$ 's capacity to capture between and within person's variability of symptoms, mood and behaviour over time.

\section{Retrospective electronic questionnaires}

Patients completed a short self-report morning and end-of-day questionnaire in the PsyMate $^{\mathrm{TM}}$ application as soon as they woke up or prior going to bed, respectively, during the same week. In these questionnaires patients retrospectively evaluated, on a 7-point Likert scale, their sleep quality during the previous night and overall experienced fatigue and mood during the preceding day, respectively (e.g. 'Overall, I felt tired today', 'Overall, I felt tense today') (Supplemental Table 7.2).

\section{Retrospective paper questionnaires}

On the last study day, patients completed a paper questionnaire that was visually identical to the electronic PsyMate ${ }^{\mathrm{TM}}$ interface, but now requested participants to retrospectively evaluate their fatigue (general, mental, physical) over the past seven days. Moreover, patients completed conventional retrospective questionnaires measuring fatigue, anxiety and depressive symptoms.

The FSS is a nine-item questionnaire assessing fatigue severity in daily life over the past week and uses a 7-point Likert scale. The total score is calculated by averaging the scores of the individual item responses. Individual item scores can range from 1-7 (no-maximum fatigue). A total FSS score of $\geq 4$ is considered as indicative of 
problematic fatigue. ${ }^{24}$ The FSS is one of the most frequently used instruments to evaluate fatigue in renal patients. ${ }^{4}$

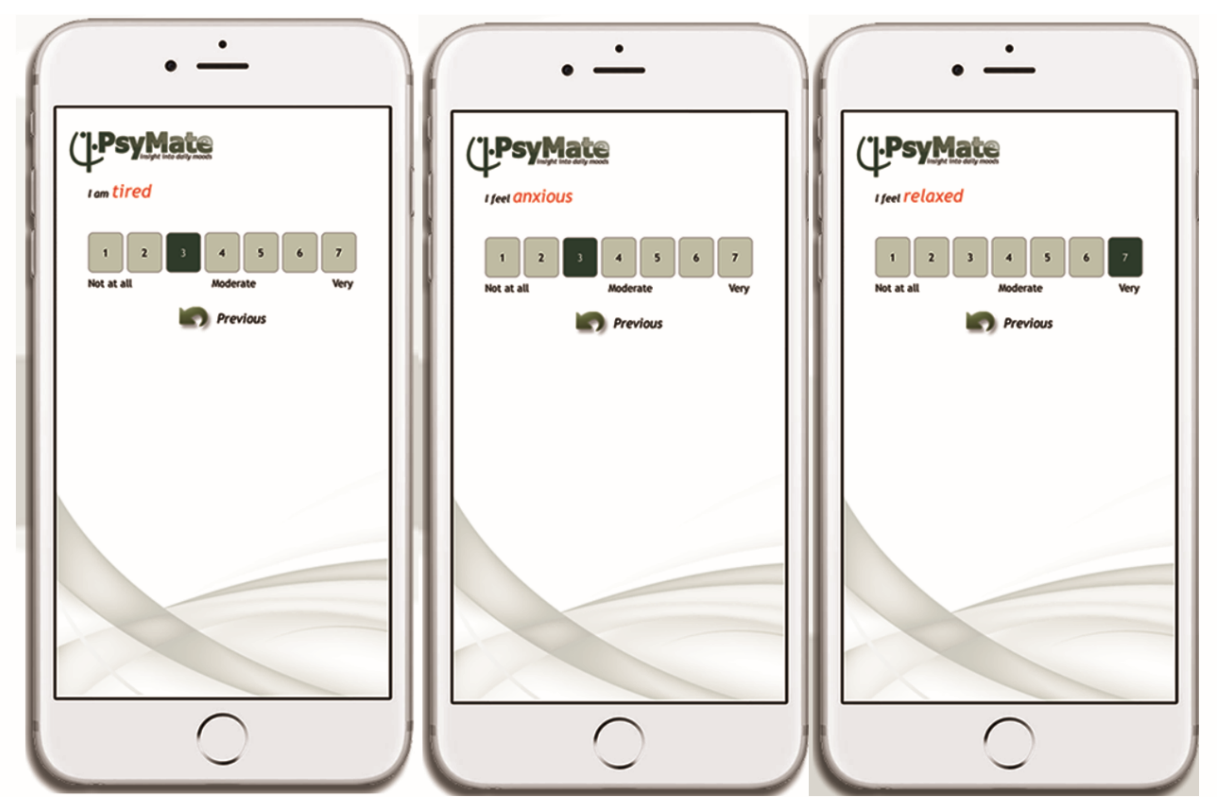

Figure 7.1 PsyMate interferace representation of experience sampling methodology questionnaire.

The HADS is developed to assess depressive symptoms and anxiety over the past week in somatically ill patients. ${ }^{25}$ Both subscales for depression and anxiety range from 0 to 21 . Scores of $\geq 8$ possibly indicate a clinical depression or anxiety disorder. The HADS has been validated in HD patients. ${ }^{26,27}$

\section{Baseline data and laboratory measurements}

Demographical, clinical and laboratory data were obtained from computerized medical records at the hospital.

\section{Haemodialysis treatment}

Conventional HD technique was used except for four patients that were on haemodiafiltration. Participants underwent dialysis treatment with bicarbonate dialysate three times weekly either in sessions during daytime (3-4 hours) or at night 
(6-7 hours). Arterial blood flow was 300-400 $\mathrm{ml} /$ minute during daytime treatment and 150-200 ml/minute during overnight dialysis. Dialysis rate flow was $400-600 \mathrm{ml} /$ minute for day treatment and $300 \mathrm{ml} /$ minute overnight. Most patients received regular treatment with recombinant human erythropoietin, antihypertensive medications, vitamin $\mathrm{D}$ analogues, phosphate and potassium binders.

\section{Study procedure}

The general procedure has been previously described in detail. ${ }^{18}$ In brief, after a briefing session, patients used the PsyMate ${ }^{\mathrm{TM}}$ application for one week to rate their momentary fatigue and mood as well as the retrospective morning and end-of-day questionnaires. Retrospective paper questionnaires were completed at the end of the study week during patients' regular HD treatment.

\section{Statistical analysis}

Statistical analysis was performed by using SPPS software, version 25. Data are reported as mean and standard deviation $(S D)$ or median and [range] as appropriate. Each participant received a maximum of 70 momentary PsyMate ${ }^{\mathrm{TM}}$ questionnaires over the entire study week (seven days of 10 beeps).

For each patient, three indices of momentary fatigue were calculated from these data: 1) the average fatigue score of all completed ESM observations to capture average momentary fatigue, 2) the average fatigue score on the last day to capture most recent momentary fatigue, and 3) the maximum fatigue score over the entire study week to capture peak momentary fatigue. The same three indices were calculated for negative affect (i.e. average score of feeling anxious, down, irritable and powerless).

To investigate differences between retrospective and momentary fatigue reports, paired samples $t$-tests were used to compare patients' average ESM fatigue score with their average retrospective end-of-day fatigue score and their retrospective end-ofweek fatigue score, respectively. To assess whether patients with an FSS or an HADS score indicative of possible clinical significant fatigue or depression or anxiety disorder, respectively, had a higher average score of momentary fatigue or negative affect, independent samples $t$-tests were used with a score of four (FSS) and eight (HADS subscale) as specified cut point. To investigate which index of momentary fatigue or negative affect best corresponded to the total score of the conventional 
retrospective FSS and HADS questionnaires, respectively, correlation analyses were carried out. Finally, linear regression analysis (backward stepwise method, 0.05 significance level) was carried out to explore potential predictors of the difference between momentary and retrospective reports.

A sample size of 34 participants was required to detect a medium or larger effect size (Cohen's $d \geq 0.50$ ) with alpha error probability of 0.05 and power of $80 \%$ regarding our first study aim. Moreover, ESM data have multilevel structure, with observations (level 1) nested within individuals (level 2). The richness of data obtained by conducting multiple observations nested within individuals allowed to carry out reliable statistical estimations in relatively small samples.

\section{Results}

\section{Sample characteristics}

Forty-two patients (33 males, 77.5\%) enrolled in this study. Two patients were excluded from the analyses conform ESM guidelines because they completed less than $30 \%$ of all PsyMate ${ }^{\mathrm{TM}}$ questionnaires. ${ }^{28}$ The mean age of our sample was 64.35 ( $S D=13.69$ ) years. Table 7.1 reports demographical and clinical characteristics. A total of 1777 ESM PsyMate ${ }^{\mathrm{TM}}$ questionnaires were completed, averaging to 44 out of 70 questionnaires per patient (63\% compliance and $97 \%$ completion rate). The average number of completed ESM questionnaires per day was 254 (range:236-300). The median time needed to complete one ESM questionnaire was 1:21 min [IQR:1:09-2:29]. One patient did not complete the end-of-day ESM questionnaires. Another patient did not return the end-of-week retrospective paper questionnaires.

\section{Momentary fatigue and mood assessments}

At the level of the ESM questionnaires, average momentary fatigue on a 1-7 scale was 3.36 ( $S D=1.97$, range:1.00-7.00). Patients responded two or higher to the general statement 'I feel tired' in 1209 completed ESM questionnaires, which means that patients indicated to experience at least some fatigue in $68 \%$ of all momentary observations. Average physical fatigue was 4.26 ( $S D=1.61$, range:1.00-7.00) and average mental fatigue was $2.71(S D=1.60$, range:1.00-7.00). The large standard 
deviations and range of observed fatigue scores indicate substantial variability in fatigue between patients. Moreover, fatigue scores also varied considerably within individuals over time. This is reflected by within-individual standard deviations (i.e. variation in the fatigue scores of the same individual) and the large variability therein.

The mean within-individual standard deviation of fatigue was 1.04 ( $S D=0.52$, range: $0.00-2.50)$. Average momentary positive and negative affect score was 5.32 ( $S D=1.30$; range:1.00-7.00), and 1.75 ( $S D=1.01$, range: $1.00-6.50)$, respectively. The mean withinindividual standard deviation for positive and negative affect was 0.67 ( $S D=0.33$, range: $0.00-2.10)$ and $0.46(S D=0.28$, range: $0.00-1.31)$, respectively. Again, this indicates that mood varied between as well as within patients. The average momentary negative affect score correlated significantly with the average momentary fatigue score, $r=0.63, p=<0.001$. Average sleep quality was 4.92 ( $S D=1.83$, range: 1.00 7.00).

\section{Retrospective fatigue and mood assessments}

Mean fatigue scores on the retrospective end-of-day and end-of-week questionnaires were $3.44(S D=1.84$, range: 1.00-7.00) and $3.82(S D=2.02$, range: 1.00-7.00), respectively. The average FSS score was 4.99 ( $S D=1.17$; range:2.10-7.00). Thirty (77\%) patients had an FSS score of four or higher, potentially indicative of clinically significant fatigue. ${ }^{24}$ The average HADS depression subscale score was 4.15 (SD=3.81, range:0.00-16.00) and $4.54(S D=3.63$, range: 0.00-14.00) for the anxiety subscale. Eight patients (21\%) scored eight or higher on the HADS depression subscale, and nine individuals (23\%) scored eight or higher on the HADS anxiety subscale, indicating the possible presence of a depressive or anxiety disorder, respectively. ${ }^{25}$ 
Table 7.1 Participants' baseline characteristics ( $\mathrm{N}=40)$.

\begin{tabular}{|c|c|}
\hline Characteristics & Mean \pm SD [range] / n (percentage) \\
\hline \multicolumn{2}{|l|}{ Demographical \& clinical characteristics } \\
\hline Age (years) & $64.4 \pm 13.7[34-84]$ \\
\hline Sex (Male) & $31(77.5 \%)$ \\
\hline $\mathrm{BMI}\left(\mathrm{Kg} / \mathrm{m}^{2}\right)$ & $27.9 \pm 5.9$ \\
\hline Charlson Comorbidity Index & $2.7 \pm 1.6[0-6]$ \\
\hline \multicolumn{2}{|l|}{ Primary kidney disease } \\
\hline Hypertension & $8(20 \%)$ \\
\hline Glomerulonefritis & $2(5 \%)$ \\
\hline Diabetes & $13(32.5 \%)$ \\
\hline Interstitial nefritis & $0(0 \%)$ \\
\hline Polycystic kidney disease & $6(15 \%)$ \\
\hline Other/unknown & $11(27.5 \%)$ \\
\hline Dialysis vintage (months) & $49 \pm 47$ \\
\hline Diuresis/ residual urine output & $14(35 \%)$ \\
\hline Diuresis/ residual urine output $(\mathrm{ml} / 24 \mathrm{~h})^{\dagger}$ & $1044 \pm 687$ \\
\hline Interdialytic weight gain $(\mathrm{Kg}){ }^{*}$ & $1.6 \pm 0.7$ \\
\hline UF rate $\mathrm{ml} / \mathrm{h} / \mathrm{lg}(n=34){ }^{*}$ & $4.35 \pm 2.23$ \\
\hline Anuric patients $(n=26)$ & $4.50 \pm 2.34$ \\
\hline Non-anuric patients $(n=8)$ & $3.87 \pm 1.93$ \\
\hline Day HD treatment $(n=25)$ & $4.82 \pm 2.34$ \\
\hline Night HD treatment $(n=9)$ & $3.06 \pm 1.28$ \\
\hline \multicolumn{2}{|l|}{ Haemodialysis access } \\
\hline Arteriovenous fistula & $30(75 \%)$ \\
\hline Arteriovenous graft & $3(7.5 \%)$ \\
\hline Central venous catheter & $7(17.5 \%)$ \\
\hline \multicolumn{2}{|l|}{ Dialysis group (dialysis hours/treatment) } \\
\hline Morning $(3.5-5.0 \mathrm{~h})$ & $18(45 \%)$ \\
\hline Afternoon $(4.0 \mathrm{~h})$ & $8(20 \%)$ \\
\hline Evening $(4.0 \mathrm{~h})$ & $4(10 \%)$ \\
\hline Night $(7-7.25$ h) & $10(25 \%)$ \\
\hline \multicolumn{2}{|l|}{ Laboratory characteristics } \\
\hline spKt/V: urea clearance & $1.6 \pm 0.3$ \\
\hline Haemoglobin (mmol/l) & $7.0 \pm 0.6$ \\
\hline Serum albumin $(g / l)$ & $39.8 \pm 3.4$ \\
\hline Serum creatinine $(\mu \mathrm{mol} / \mathrm{l})$ & $922.8 \pm 260.8$ \\
\hline Serum urea $(\mathrm{mmol} / \mathrm{l})$ & $23.6 \pm 4.9$ \\
\hline Parathyroid hormone (pmol/l) & $37.8 \pm 26.6$ \\
\hline Calcium (mmol/l) & $2.3 \pm 0.1$ \\
\hline Phosphorus (mmol/l) & $1.7 \pm 0.5$ \\
\hline C-reactive protein $(\mathrm{mg} / \mathrm{l})$ & $9.4 \pm 9.8$ \\
\hline
\end{tabular}

${ }^{\dagger}$ excluding anuric patients $(n=26),{ }^{*}$ In patients with target weight $(n=34)$, * ${ }^{*}$ excluding patients without ultrafiltration (UF) 


\section{Comparison between momentary ratings and retrospective questionnaires scores}

Retrospective end-of-week fatigue scores $(M=3.82, S E=0.32)$ were, on average, higher than the mean of patients' momentary fatigue scores during the preceding study week $(M=3.31, S E=0.26)$. This difference of $0.51(95 \% \mathrm{Cl}[0.22,0.80])$ was statistically significant $t(38)=3.54, p=0.001$, and represented an effect size of $d=0.57$. The average retrospective end-of-day fatigue score for each individual $(M=3.42, S E=0.24)$ was also higher than the average momentary fatigue scores during the entire study week $(M=3.29, S E=0.16)$. However, this difference of 0.13 (95\% Cl $[-0.12,0.38])$ was not significant, $t(38)=1.07, p=0.291, d=0.17$.

Patients with an FSS $\geq 4$ had an average momentary fatigue score over the entire study week $(M=3.64, S E=0.31)$ that was significantly higher compared to subjects with an FSS score of $<4(M=2.22, S E=0.25), t(31.457)=3.56, p=0.001,95 \% \mathrm{Cl}[0.61,2.24]$.

Patients with a HADS depression subscale of $\geq 8$ had an average negative affect score over the entire study week $(M=2.74, S E=0.24)$ that was significantly higher compared to patients with a HADS depression score of $<8(M=1.46, S E=0.12), t(10.808)=4.67$ $p=0.001,95 \% \mathrm{Cl}[0.67,1.87]$. Likewise, patients with a HADS anxiety subscale of $\geq 8$ had an average negative affect score over the entire study week $(M=2.75, S E=0.30)$ that was significantly higher compared to individuals with a HADS anxiety score of $<8$ $(M=1.42, S E=0.10), t(9.826)=4.20 p=0.002,95 \% \mathrm{Cl}[0.62,2.04]$.

\section{ESM indices and retrospective questionnaires}

Table 7.2 reports correlations between the conventionally used retrospective FSS and HADS and the ESM indices of momentary fatigue and negative affect, respectively. Correlations are strongest for the average of all momentary fatigue and negative affect scores.

\section{Potential predictors of the fatigue memory-experience gap}

The difference between end-of-week fatigue ratings and the average of momentary fatigue scores during the preceding study week did not significantly correlate with the average of ESM negative affect scores during the study week, $r=0.09, p=0.583$. In a multiple linear regression analysis, neither maximum or average ESM negative affect 
score, nor maximum or average ESM fatigue score, gender or age predicted the difference between retrospective end-of-week fatigue score and the average of momentary fatigue scores (all $p^{\prime} s>0.05$ ).

Table 7.2 Pearson correlations ( $r$ ) between total scores of the Fatigue Severity Scale (FSS) and Hospital Anxiety and Depression Scale (HADS) on the one hand, and indices for real-time momentary fatigue and negative affect feelings on the other hand, derived from data collected using Experience Sampling Methodology (ESM) ( $N=39)$.

\begin{tabular}{lcccc}
\hline Questionnaire & $r$ & Average ESM fatigue & $\begin{array}{c}\text { Average ESM } \\
\text { fatigue last day }\end{array}$ & Maximum ESM fatigue \\
\hline FSS & $C l$ & 0.66 & 0.65 & 0.53 \\
& $p$ & {$[0.49,0.80]$} & {$[0.47,0.80]$} & {$[0.28,0.71]$} \\
& $N$ & 39 & $<0.001$ & 0.001 \\
Questionnaire & & Average ESM & Average ESM & Maximum ESM \\
& & negative affect & negative affect last day & negative effect \\
\hline HADS & $r$ & & & \\
Depression & $C l$ & {$[0.57,0.89]$} & 0.74 & 0.59 \\
subscale & $p$ & $<0.001$ & {$[0.57,0.88]$} & {$[0.36,0.79]$} \\
& $N$ & 39 & $<0.001$ & $<0.001$ \\
& & & 39 & 39 \\
& $r$ & 0.77 & 0.75 & 0.68 \\
Anxiety & $C l$ & {$[0.55,0.90]$} & {$[0.52,0.89]$} & {$[0.41,0.83]$} \\
subscale & $p$ & $<0.001$ & $<0.001$ & $<0.001$ \\
\hline
\end{tabular}

$\mathrm{Cl}=$ Bias corrected and accelerated bootstrap $95 \%$ confidence intervals.

\section{Discussion}

This is the first study investigating to what extent retrospective reports for fatigue and mood in HD patients correspond to their moment-to-moment and day-to-day symptom experiences assessed with an Experience Sampling Method in an mHealth application.

First, our results may suggest that HD patients' retrospective evaluation of fatigue symptoms overestimates their real-time symptom experience. These results are in line with previous studies reporting a memory-experience gap for symptoms, such as fatigue and pain, in other clinical populations. ${ }^{9,11-14}$ Our results showed that the memory-experience gap increased as time passed between retrospective symptom evaluation and actual symptom experience. This accords with the theoretical position that momentary assessments and retrospective memory reports may draw 
information from different sources, resulting in potentially discrepant response patterns. Momentary symptom assessments may primarily reflect information from an 'experiencing self' in a 'here-and-now' context. ${ }^{7}$ In contrast, retrospective reports rely on information from our autobiographical episodic memory, where experiences from a proximal past are processed. It has been suggested that information from this 'remembering self' may prioritize functionality over accuracy in reflecting on past experience. ${ }^{29}$ For instance, remembering situations in which fatigue was higher or at its peak may be more useful (or functional) than remembering every single moment of a certain experience. As such, autobiographical memories of symptom experience may be biased to experiences that are more relevant to guide future behaviour, rather than accurately represent the past experience. ${ }^{29}$ Furthermore, information about experiences in a more distal past may be influenced by semantic memory, which reflects personal beliefs and views on health and disease in general (e.g. 'I am always tired and there is nothing I can do about it'). ${ }^{7}$ Understanding how symptom experiences are processed and consolidated in memory may help to elucidate the discrepancies observed between momentary and retrospective reports of symptom experience. The memory-experience gap for fatigue symptoms observed in this study sample was not related to negative affect or cognitive heuristics, such as a peak-end effect. Neither could fatigue severity, negative affect, age nor gender predict the memory-experience gap for fatigue in this HD population. Future research might consider investigating the relationship with cognitive capacity or catastrophizing about symptoms. ${ }^{30}$ However, to the extent that momentary assessments and retrospective memory reports do indeed reflect information from different sources (i.e. experiencing versus remembering self) which may have a different function (e.g. accuracy versus guidance of future behaviour) explaining discrepancies between memory and experience may not be necessary all together. Alternatively, it is possible that participants completed less surveys on days or moments when they felt worse, due to the potential burden associated with completing multiple surveys a day. This might then result in an underestimation of symptom severity based on ESM reports. Future ESM studies could benefit from the inclusion of a post-study interview to assess this potential bias.

Second, correlation coefficients of the FSS and HADS with the averages of momentary assessments of fatigue and mood ranged from .66 to .77. These correlations show that conventionally used retrospective measurement instruments are moderately to 
strongly related to daily life experience of fatigue and mood in HD patients. However, we found that momentary fatigue and mood scores varied between as well as within individuals over time. This within-individual variability in fatigue and mood over time cannot be captured by conventional retrospective questionnaires. Nevertheless, this type of information may be especially relevant to design and implement personalized interventions. That is, insight in diurnal symptom patterns and factors related to these patterns may be crucial for effective treatment of fatigue. For instance, fatigue may be lower during certain types of activities for some individuals but higher for others, or may be temporally related to changes in mood for some but not for others. Such highly personalized information provided by ESM may be crucial for successful alleviation of symptoms. For instance, in a recently conducted randomized controlled trial in depressed outpatients, ESM-derived personalized feedback led to a significant and clinically relevant decrease of depressive symptoms. ${ }^{31}$

Taken together, momentary and retrospective symptom assessments may reflect different types of information. Therefore, they may complement rather than replace each other and can be used for different types of outcomes. For instance, diagnostic decision making and treatment follow-up may benefit more from ESM based selfreports as they enable to capture moment-to-moment symptom variation and provide insights in related contextual variables (e.g. location, activity, company, time of day), which may be used as entry points for tailored and personalized interventions. In contrast, retrospective symptom assessment may be more relevant when the focus of interest is understanding treatment adherence or patients' illness behaviour. ${ }^{7,32}$

Major strengths of this study include the use of an mHealth application to collect reliable and ecologically valid data, with a fifteen minutes time-frame to control for faked compliance and reduce potential recall bias; and the comparison of momentary assessments with an mHealth ESM and conventionally used retrospective questionnaires. Limitations include a potential selection bias due to a certain level of cognitive capacities needed to use the mHealth application. Moreover, careful item selection and reduction of questions may further reduce patients' burden. Promisingly, preliminary work in order to develop a valid and reliable patient-reported outcome measure (PROM) for fatigue in HD patients that takes into account different dimensions of fatigue in HD patients has been done in 2016 by the Standardized Outcomes in Nephrology (SONG) initiative's working group for fatigue in HD 
patients. ${ }^{33}$ When such a PROM would be incorporated in an mHealth ESM, it may have the potential to become a valid and reliable measurement instrument to accurately assess fatigue in HD patients. As such, it may provide concrete entry points to develop personalized and targeted interventions to alleviate fatigue symptoms and improve HD patients' quality of life. Finally, this study may have a restricted representativeness due to the imbalance between male and female participants, notwithstanding a gender imbalance of $60 \%$ men receiving HD in European countries, including the Netherlands. $^{34}$

In conclusion, we found that retrospective symptom evaluation led to higher fatigue reporting relative to real-time momentary fatigue experiences. Moreover, momentary assessments of fatigue and mood revealed important variance in symptoms between individuals and within individuals (over time), which cannot be captured by conventional retrospective measurement instruments. Therefore, we recommend that future research investigating fatigue in HD patients and variability therein in daily life should make use of the ESM rather than - or at least in addition to - retrospective measurement instruments. 


\section{Supplemental material}

Table S7.1 ESM PsyMate: questions.

\begin{tabular}{|c|c|c|}
\hline Domain & Item & Scale type \\
\hline \multirow[t]{9}{*}{ Mood } & 1. I feel cheerful & 7-point Likert scale (1 "not at all” to 7 "very much") \\
\hline & 2. I feel relaxed & 7-point Likert scale (1 "not at all" to 7 "very much") \\
\hline & 3. I feel satisfied & 7-point Likert scale (1 "not at all” to 7 "very much") \\
\hline & 4. I feel anxious & 7-point Likert scale (1 “not at all” to 7 "very much") \\
\hline & 5. I feel down & 7-point Likert scale (1 “not at all” to 7 "very much") \\
\hline & 6. I feel confident & 7-point Likert scale (1 “not at all” to 7 "very much") \\
\hline & 7. I feel irritable & 7-point Likert scale (1 "not at all” to 7 "very much") \\
\hline & 8. I feel powerless & 7-point Likert scale (1 “not at all” to 7 "very much") \\
\hline & 9. I am worrying & 7-point Likert scale (1 "not at all” to 7 "very much") \\
\hline \multirow{7}{*}{$\begin{array}{l}\text { Context } \\
\text { activity }\end{array}$} & 10. What am I doing & Doing nothing, resting, undergoing HD treatment, watching \\
\hline & (just before the beep)? & $\begin{array}{l}\text { TV, reading, listening to music, internet or social media, work, } \\
\text { household, self care, eating or drinking, having a } \\
\text { conversation, relaxing, something else }\end{array}$ \\
\hline & 11. And also? & $\begin{array}{l}\text { resting, undergoing HD treatment, watching TV, reading, } \\
\text { listening to music, internet or social media, work, household, } \\
\text { self care, eating or drinking, having a conversation, relaxing, } \\
\text { something else }\end{array}$ \\
\hline & 12. And...? & $\begin{array}{l}\text { resting, undergoing HD treatment, watching TV, reading, } \\
\text { listening to music, internet or social media, work, household, } \\
\text { self care, eating or drinking, having a conversation, relaxing; } \\
\text { something else }\end{array}$ \\
\hline & 13. I can do this well & 7-point Likert scale (1 "not at all” to 7 "very much") \\
\hline & 14. This is difficult for me & 7-point Likert scale (1 "not at all" to 7 "very much") \\
\hline & 15. I would rather be doing something else & 7-point Likert scale (1 "not at all" to 7 "very much") \\
\hline $\begin{array}{l}\text { Context } \\
\text { location }\end{array}$ & 16. Where am I? & $\begin{array}{l}\text { At the HD unit, at home, at family's/friend's place, at work, } \\
\text { public space, transport, somewhere else }\end{array}$ \\
\hline \multirow[t]{3}{*}{$\begin{array}{l}\text { Context } \\
\text { social }\end{array}$} & 17. Who am I with? & $\begin{array}{l}\text { No one, healthcare professional, partner, family living in, } \\
\text { family out-living, friends, colleagues, acquaintances, } \\
\text { strangers }\end{array}$ \\
\hline & 18. And also? & $\begin{array}{l}\text { healthcare professional, partner, family living in, family out- } \\
\text { living, friends, colleagues, acquaintances, strangers }\end{array}$ \\
\hline & 19. And...? & $\begin{array}{l}\text { healthcare professional, partner, family living in, family out- } \\
\text { living, friends, colleagues, acquaintances, strangers }\end{array}$ \\
\hline \multirow[t]{4}{*}{ Fatigue } & 20. I feel tired & 7-point Likert scale (1 "not at all” to 7 "very much") \\
\hline & Branched questions when feeling tired $>1$ & \\
\hline & 20a. I feel mentally tired & 7-point Likert scale (1 "not at all" to 7 "very much") \\
\hline & 20b. I feel physically tired & 7-point Likert scale (1 "not at all" to 7 "very much") \\
\hline \multirow[t]{7}{*}{ Physical } & 21. I am suffering from a headache & 7-point Likert scale (1 "not at all" to 7 "very much") \\
\hline & 22. I am suffering from muscle cramps & 7-point Likert scale (1 "not at all" to 7 "very much") \\
\hline & 23. I am suffering from pain in bones or joints & 7-point Likert scale (1 "not at all" to 7 "very much") \\
\hline & 24. I am suffering from itch & 7-point Likert scale (1 "not at all" to 7 "very much") \\
\hline & 25. I am suffering from restless legs & 7-point Likert scale (1 "not at all" to 7 "very much") \\
\hline & 26. I have been physically active before the beep & 7-point Likert scale (1 "not at all" to 7 "very much") \\
\hline & 27. I have been mentally active before the beep & 7-point Likert scale (1 "not at all" to 7 "very much") \\
\hline General & 28. This beep disturbed me & 7-point Likert scale (1 "not at all" to 7 "very much") \\
\hline
\end{tabular}

Note: Questions about momentary activities, contextual aspects and physical symptoms have been discussed in the previous chapter no 6. 
Table S7.2 ESM PsyMate: retrospective questionnaires.

Morning questionnaire

\begin{tabular}{|c|c|c|}
\hline Domain & Item & Scale type \\
\hline \multirow{4}{*}{$\begin{array}{l}\text { Sleep } \\
\text { and night } \\
\text { rest }\end{array}$} & 1. I slept well & 7-point Likert scale (1 "not at all" to 7 "very good") \\
\hline & $\begin{array}{l}\text { 2. How long did it take before I felt asleep } \\
\text { yesterday evening }\end{array}$ & $\begin{array}{l}0-5 \mathrm{~min}, 5-15 \mathrm{~min}, 15-30 \mathrm{~min}, 30-45 \mathrm{~min}, 45 \mathrm{~min}- \\
1 \text { hour, } 1-2 \text { hours, } 2-4 \text { hours, }>4 \text { hours }\end{array}$ \\
\hline & 3. How often did I wake up last night & 1 time, 2 times, 3 times, 4 times, 5 times, $>5$ times \\
\hline & 4. I feel rested & 7-point Likert scale (1 "not at all" to 7 "very much") \\
\hline \multirow[t]{5}{*}{ Fatigue } & 5. I feel tired & 7-point Likert scale (1 “not at all” to 7 "very much") \\
\hline & Branched questions when feeling tired $>1$ & \\
\hline & 5a. I feel mentally tired & 7-point Likert scale ( 1 "not at all" to 7 "very much") \\
\hline & 5b. I feel physically tired & 7-point Likert scale ( 1 "not at all" to 7 "very much") \\
\hline & 6. I do not look forward to this day & 7-point Likert scale (1 "not at all" to 7 "very much") \\
\hline \multicolumn{3}{|c|}{ End-of-day questionnaire } \\
\hline Domain & Item & Scale type \\
\hline \multirow[t]{2}{*}{ General } & 1. Today was an ordinary day & 7-point Likert scale (1 “not at all” to 7 "very much”) \\
\hline & $\begin{array}{l}\text { 2. Without the application I would have } \\
\text { done other things today }\end{array}$ & 7-point Likert scale (1 "not at all" to 7 "very much") \\
\hline \multirow[t]{3}{*}{ Fatigue } & 3. Overall, I felt tired today & 7-point Likert scale (1 “not at all” to 7 "very much") \\
\hline & 4. Overall, I felt mentally tired today & 7-point Likert scale ( 1 "not at all" to 7 "very much") \\
\hline & 5. Overall, I felt physically tired today & 7-point Likert scale (1 "not at all" to 7 "very much") \\
\hline \multirow[t]{3}{*}{ Mood } & 6. Overall, I felt good today & 7-point Likert scale (1 "not at all" to 7 "very much") \\
\hline & 7. Overall, I felt tense today & 7-point Likert scale (1 "not at all" to 7 "very much") \\
\hline & 8. Overall, I've been worrying today & 7-point Likert scale ( 1 "not at all" to 7 "very much") \\
\hline Medication & 9. Today, I took my medication & 7-point Likert scale ( 1 "nothing" to 7 "everything") \\
\hline
\end{tabular}




\section{References}

1. Jhamb M, Argyropoulos C, Steel JL, et al. Correlates and outcomes of fatigue among incident dialysis patients. Clinical journal of the American Society of Nephrology : CJASN. 2009;4(11):1779-1786.

2. Urquhart-Secord R, Craig JC, Hemmelgarn B, et al. Patient and Caregiver Priorities for Outcomes in Hemodialysis: An International Nominal Group Technique Study. American journal of kidney diseases : the official journal of the National Kidney Foundation. 2016;68(3):444-454.

3. Jhamb M, Weisbord SD, Steel JL, Unruh M. Fatigue in patients receiving maintenance dialysis: a review of definitions, measures, and contributing factors. American journal of kidney diseases : the official journal of the National Kidney Foundation. 2008;52(2):353-365.

4. Artom M, Moss-Morris R, Caskey F, Chilcot J. Fatigue in advanced kidney disease. Kidney international. 2014;86(3):497-505.

5. Brys $A D H$, Lenaert B, Van Heugten CM, Gambaro G, Bossola M. Exploring the Diurnal Course of Fatigue in Patients on Hemodialysis Treatment and Its Relation With Depressive Symptoms and Classical Conditioning. J Pain Symptom Manage. 2019;57(5):890-898.e894.

6. Stone AA, Shiffman S, Schwartz JE, Broderick JE, Hufford MR. Patient compliance with paper and electronic diaries. Controlled clinical trials. 2003;24(2):182-199.

7. Van den Bergh O, Walentynowicz M. Accuracy and bias in retrospective symptom reporting. Current opinion in psychiatry. 2016;29(5):302-308.

8. Walentynowicz M, Bogaerts K, Van Diest I, Raes F, Van den Bergh O. Was it so bad? The role of retrospective memory in symptom reporting. Health psychology : official journal of the Division of Health Psychology, American Psychological Association. 2015;34(12):1166-1174.

9. Schneider S, Stone AA, Schwartz JE, Broderick JE. Peak and end effects in patients' daily recall of pain and fatigue: a within-subjects analysis. The journal of pain : official journal of the American Pain Society. 2011;12(2):228-235.

10. Shiffman S, Stone AA, Hufford MR. Ecological momentary assessment. Annual review of clinical psychology. 2008;4:1-32.

11. Lenaert B, Neijmeijer M, van Kampen N, van Heugten C, Ponds R. Poststroke Fatigue and Daily Activity Patterns During Outpatient Rehabilitation: An Experience Sampling Method Study. Archives of physical medicine and rehabilitation. 2020;101(6):1001-1008.

12. Mujagic Z, Leue C, Vork L, et al. The Experience Sampling Method--a new digital tool for momentary symptom assessment in IBS: an exploratory study. Neurogastroenterology and motility : the official journal of the European Gastrointestinal Motility Society. 2015;27(9):1295-1302.

13. Ben-Zeev D, McHugo GJ, Xie H, Dobbins K, Young MA. Comparing retrospective reports to realtime/real-place mobile assessments in individuals with schizophrenia and a nonclinical comparison group. Schizophrenia bulletin. 2012;38(3):396-404.

14. Friedberg F, Sohl SJ. Memory for fatigue in chronic fatigue syndrome: the relation between weekly recall and momentary ratings. International journal of behavioral medicine. 2008;15(1):29-33.

15. Abdel-Kader K, Jhamb M, Mandich LA, et al. Ecological momentary assessment of fatigue, sleepiness, and exhaustion in ESKD. BMC nephrology. 2014;15:29.

16. Riis J, Loewenstein G, Baron J, Jepson C, Fagerlin A, Ubel PA. Ignorance of hedonic adaptation to hemodialysis: a study using ecological momentary assessment. Journal of experimental psychology. General. 2005;134(1):3-9.

17. Horigan AE, Barroso JV. A Comparison of Temporal Patterns of Fatigue in Patients on Hemodialysis. Nephrology nursing journal : journal of the American Nephrology Nurses' Association. 2016;43(2):129138, 148; quiz 139. 
18. Brys A, Stifft F, van Heugten C, Bossola M, Gambaro G, Lenaert B. mHealth based Experience Sampling Method to identify fatigue in the context of daily life in hemodialysis patients. Clinical Kidney Journal. 2020; Sep 01. Online ahead of print.

19. Van Diest I, De Peuter S, Eertmans A, Bogaerts K, Victoir A, Van den Bergh O. Negative affectivity and enhanced symptom reports: differentiating between symptoms in men and women. Social science \& medicine (1982). 2005;61(8):1835-1845.

20. Myin-Germeys I, Birchwood M, Kwapil T. From environment to therapy in psychosis: a real-world momentary assessment approach. Schizophrenia bulletin. 2011;37(2):244-247.

21. Lenaert B, Colombi M, van Heugten C, Rasquin S, Kasanova Z, Ponds R. Exploring the feasibility and usability of the experience sampling method to examine the daily lives of patients with acquired brain injury. Neuropsychological rehabilitation. 2019;29(5):754-766.

22. Wichers $M$, Peeters $F$, Rutten BP, et al. A time-lagged momentary assessment study on daily life physical activity and affect. Health psychology : official journal of the Division of Health Psychology, American Psychological Association. 2012;31(2):135-144.

23. Oorschot $M$, Lataster $T$, Thewissen $V$, et al. Emotional experience in negative symptoms of schizophrenia--no evidence for a generalized hedonic deficit. Schizophrenia bulletin. 2013;39(1):217225.

24. Krupp LB, LaRocca NG, Muir-Nash J, Steinberg AD. The fatigue severity scale. Application to patients with multiple sclerosis and systemic lupus erythematosus. Archives of neurology. 1989;46(10):11211123.

25. Zigmond AS, Snaith RP. The hospital anxiety and depression scale. Acta psychiatrica Scandinavica. 1983;67(6):361-370.

26. Loosman WL, Siegert CE, Korzec A, Honig A. Validity of the Hospital Anxiety and Depression Scale and the Beck Depression Inventory for use in end-stage renal disease patients. The British journal of clinical psychology. 2010;49(Pt 4):507-516.

27. Untas A, Aguirrezabal M, Chauveau P, Leguen E, Combe C, Rascle N. [Anxiety and depression in hemodialysis: validation of the Hospital Anxiety and Depression Scale (HADS)]. Nephrologie \& therapeutique. 2009;5(3):193-200.

28. Delespaul P.A.E.G. Assessing Schizophrenia in Daily Life: The Experience Sampling Method, Maastricht University; 1995.

29. Conway MA, Pleydell-Pearce CW. The construction of autobiographical memories in the self-memory system. Psychological review. 2000;107(2):261-288.

30. Lukkahatai N, Saligan LN. Association of catastrophizing and fatigue: a systematic review. J Psychosom Res. 2013;74(2):100-109.

31. Kramer I, Simons CJ, Hartmann JA, et al. A therapeutic application of the experience sampling method in the treatment of depression: a randomized controlled trial. World psychiatry : official journal of the World Psychiatric Association (WPA). 2014;13(1):68-77.

32. Horne R, Chapman SC, Parham R, Freemantle N, Forbes A, Cooper V. Understanding patients' adherence-related beliefs about medicines prescribed for long-term conditions: a meta-analytic review of the Necessity-Concerns Framework. PloS one. 2013;8(12):e80633.

33. Nair D, Finkelstein FO. Toward Developing a Patient-Reported Outcome Measure for Fatigue in Hemodialysis. American journal of kidney diseases : the official journal of the National Kidney Foundation. 2019;74(2):151-154.

34. Kramer A, Pippias M, Noordzij M, et al. The European Renal Association - European Dialysis and Transplant Association (ERA-EDTA) Registry Annual Report 2015: a summary. Clin Kidney J. 2018;11(1):108-122. 



\section{Chapter 8}

General discussion 
Chapter 8 


\section{General discussion}

Fatigue, commonly defined as an overwhelming, debilitating and sustained sense of exhaustion, ${ }^{1}$ is the most frequently reported complaint of haemodialysis (HD) patients and impairs their health-related quality of life (HrQoL) considerably. ${ }^{2,3}$ As such, it is one of HD patients' most prioritized outcomes. ${ }^{4}$ However, the development of effective therapies for fatigue in HD patients is complicated by the lack of knowledge about the underlying aetiology and the absence of an appropriate measurement instrument taking into account the multifactorial nature of fatigue in HD patients. Therefore, the main aims of this thesis were to advance our understanding of fatigue in HD patients, and to investigate whether a mobile health (mHealth) Experience Sampling Methodology (ESM) may be a suitable measurement method to unravel the multifactorial nature of fatigue in HD patients. In Part I of this thesis we investigated whether fatigue in HD patients may be related to inflammation. In part II and III of this thesis we explored to what extent psychological, social, behavioural and contextual factors may be related to fatigue in HD patients. In Part III of this thesis we examined the added value of the mHealth ESM as a novel measurement technology to assess fatigue in HD patients. In this last chapter, the main findings of this thesis will be highlighted and discussed from a broader scientific and clinical perspective. Moreover, methodological considerations and implications for clinical practice will be discussed. Finally, new directions for future research will be formulated.

\section{The role of inflammation}

In the first part of this thesis (chapter $\mathbf{2}$ and $\mathbf{3}$ ), the hypothesis of inflammationinduced fatigue in HD patients was studied. According to this hypothesis, disease-

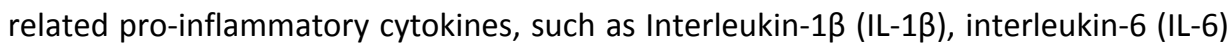
and tumour necrosis factor $\alpha$ (TNF- $\alpha$ ), may interact with the central nervous system (CNS) and cause sickness behaviour, including fatigue and resting. ${ }^{5}$ Chronic inflammation is common in the HD population ${ }^{6}$ and research in other medical conditions, such as chronic fatigue syndrome (CFS), ${ }^{7}$ multiple sclerosis (MS), ${ }^{8,9}$ rheumatoid arthritis $(\mathrm{RA})^{10,11}$ and cancer, $^{12,13}$ already showed a link between inflammation and fatigue symptoms. In this thesis, we showed that HD patients' general fatigue experience correlated significantly with inflammatory parameters (i.e. 
serum endotoxin and IL-6 levels) (chapter 2). However, we did not find a link between inflammatory parameters (i.e. IL-1 3 , IL- 6 and TNF- $\alpha$ ) and fatigue occurring immediately after the HD session (i.e. postdialysis fatigue (PDF)) (chapter 3). These findings are in line with a limited number of previous studies investigating the relationship between inflammation and general fatigue or PDF in HD patients. ${ }^{14-17}$ It may well be that general fatigue and PDF in HD patients result from different biological pathways, with the inflammation-induced fatigue hypothesis solely holding true for HD patients' general fatigue experience. It is possible that the altered inflammatory state in HD patients only promotes fatigue in the long term but not in the short term. In contrast, it might be hypothesized that PDF is rather a response to physical or psychological stress associated with the HD procedure than the result of an inflammatory response. In breast cancer patients, cancer-related fatigue has been associated with cortisol dysregulation ${ }^{18}$ and disrupted circadian cortisol rhythms have been demonstrated in HD patients. ${ }^{19}$ However, cautions is required when putting forward these conjectures, as PDF's transient nature (i.e. a fatigue sensation specifically occurring after the HD procedure without chronic persistence) complicates a head-to-head comparison with (chronic) fatigue in other medical conditions.

It is clear that additional research is needed to determine whether inflammation and general fatigue in HD patients are causally related and whether this may offer entry points for future therapies. In patients with inflammatory diseases, such as RA and systematic lupus erythematosis, previous studies found that treatment with biological agents interfering with pro-inflammatory cytokine signalling (e.g. IL-6 and TNF- $\alpha$ blocking agents) resulted in a significant relief from fatigue. ${ }^{20-24}$ However, in these populations, biological agents are administered legitimately as part of patients' conventional therapy. As long as a causal relationship between inflammation and general fatigue in HD patients is not scientifically proven, there is no evidence to consider therapies based on biological agents as a treatment option for general fatigue in HD patients. Moreover, it can be argued that too much weight is given to the biological determinants of fatigue in HD patients. Results from our multiple regression analysis in chapter 2 revealed that 31\% of the variance in general fatigue experience in HD patients was explained by inflammation and depressed mood, indicating that fatigue may be only partially accounted for by inflammation. Moreover, the majority of the variance in general fatigue remained unexplained. This suggests that other determinants besides inflammation are involved in the 
development and maintenance of fatigue in HD patients. Non-biological factors that contribute to fatigue in HD patients merit equal attention and may offer new perspectives for therapeutic strategies.

\section{A biopsychosocial approach to fatigue in chronic HD patients}

In this thesis, we demonstrated that fatigue in chronic HD patients is not a static but a fluctuating symptom, which seems to be related to concurrent psychological and contextual factors in patients' daily life. Indeed, our findings showed that fatigue severity varied between as well as within HD patients over time (chapter $\mathbf{4}$ and 6), and was related to HD patients' concurrent mood and contextual factors, such as daily activity, social company, location or sleep quality (chapter $\mathbf{4}$ and 6). More specifically, when patients reported negative affect (i.e. feeling anxious, down, irritable or powerless), they concurrently experienced more fatigue. In contrast, they reported to be less fatigued when feeling cheerful, relaxed, satisfied or confident at the same time. Similarly, fatigue experience during certain activities, such as work or household activities, was less severe relative to relaxing or resting, whereas undergoing HD treatment was experienced as equally tiring. Interestingly, time-lagged analyses revealed that relaxing was a behavioural response to fatigue and that feeling down may be secondary to fatigue in the short term (chapter 6). Further, chronic depressed mood was associated with a steadier but more severe diurnal fatigue pattern, and non-depressed patients showed a more fluctuating, though less severe diurnal fatigue pattern (chapter 4). It can be hypothesized that when depressed mood is present for a longer period of time, it may be associated with a more chronic fatigue experience in HD patients. Finally, results from the same study showed that fatigue in HD patients may - in part - be regarded as a learned response to the hospital environment, as fatigue scores reported at the HD unit prior to the HD treatment were significantly higher relative to fatigue scores reported in other environments that have not been paired with HD treatment (chapter 4).

Taken together, these findings support the importance of psychological and contextual factors in the development and maintenance of fatigue in HD patients. Therefore, reducing fatigue in HD patients to an exclusively biologically induced phenomenon detracts from its multifactorial nature. Earlier studies on fatigue in other 
medical conditions, such as CFS and MS patients, incorporated both physiological and psychological factors in aetiological models of fatigue. ${ }^{25,26}$ They identified that cognitive and behavioural factors, such as perceptions surrounding fatigue and coping behaviours, contribute to the experience of fatigue. ${ }^{27}$ It can be argued that a similar approach to fatigue in HD patients, integrating biological, psychological and social factors, does more justice to its complex nature. A recently published systematic review, investigating the role of psychological factors in fatigue among end-stage kidney disease patients reached a similar conclusion and proposed a biopsychosocial model to explain fatigue in HD patients. ${ }^{28}$ In this model, fatigue may initially be triggered by physiological imbalances due to renal failure (e.g. inflammation) or stress. At this stage, fatigue may still function as an adaptive response to induce rest in order to conserve energy and allow recovery to take place. However, the experience of fatigue may subsequently be maintained by psychological and social factors and result in a vicious cycle of negative beliefs (e.g. fearful thoughts or catastrophizing about the consequences of fatigue), depression and anxiety, and maladaptive behavioural patterns such as all-or-nothing behaviour or avoidance of physical or mental activity.

Moreover, findings from chapter $\mathbf{7}$, showing a discrepancy between fatigue reporting based on retrospective and momentary fatigue assessments, provide additional evidence for the role of cognitive factors (e.g. memory processes, personal beliefs about fatigue) in the experience of fatigue in HD patients. Retrospective fatigue assessments may be influenced by the semantic memory, reflecting personal beliefs and views on health and disease in general, and may result in overestimation of experienced fatigue severity. ${ }^{29}$

In conclusion, based on the results of the discussed chapters, it seems evident that psychological, social and contextual factors play a role in the development and maintenance of fatigue in HD patients above and beyond biological factors. In this regard, intervention studies focussing on psychological and contextual determinants of fatigue in HD patients may have beneficial effects. For instance, cognitive behavioural therapy (CBT) has successfully been applied to alleviate fatigue symptoms in other chronic conditions, including $\mathrm{MS}$ and cancer. $^{30,31}$ During CBT, factors maintaining fatigue, such as negative beliefs and maladaptive behaviours, are challenged and replaced with more adaptive beliefs and behaviours. Additionally, CBT may include elements such as stress management, mindfulness or relaxation techniques in order to promote resilience. ${ }^{32}$ Recently, a pilot study in ten HD patients 
demonstrated the feasibility of an eight week during CBT-intervention on symptoms, including fatigue. ${ }^{33}$ However, no significant changes in symptom experience were found, which may have been due to the small sample size $(N=10)$. More research is warranted to assess whether CBT may be an effective non-pharmacological therapeutic strategy to tackle fatigue in HD patients. A promising randomized controlled trial in which a 4-6 weeks lasting CBT-based intervention was conducted to target HD patients' fatigue beliefs and behaviours in order to facilitate coping, has recently been completed but the results have not been published yet. ${ }^{32}$

\section{Added value of an mHealth ESM-based fatigue measurement instrument}

From the previous section it becomes clear that in order to advance our understanding of fatigue in HD patients, it is indispensable to take into account psychological and contextual factors that may be related to fatigue in daily life. We also showed that fatigue in HD patients varied over time and that conventional retrospective paper reports (e.g. the fatigue severity scale (FSS)) only correlated moderately with 'real-time' fatigue as assessed by means of a fatigue paper diary (chapter 4). Hence, conventional retrospective fatigue reports do not seem to be the most reliable measurement instrument to assess fatigue in HD patients, as they do not allow assessing symptom variability and related factors. ${ }^{34}$ Therefore, we introduced a novel measurement technology: a mobile health (mHealth) Experience Sampling Methodology (ESM). The mHealth ESM enabled us to capture the diurnal fluctuations of fatigue in HD patients, by repeatedly assessing fatigue severity in 'realtime' (chapter 6). Moreover, it allowed us to identify psychological (e.g. negative affect) and contextual (e.g. undergoing HD treatment) factors related to this variability, by assessing fatigue in patients' daily life, for instance at home or at work and during daily activities (chapter 6). Besides providing detailed insight in the variability of fatigue and related factors, an ESM-based fatigue measurement instrument has the advantage of overcoming recall and ecological bias that conventional retrospective paper questionnaires are liable to. ${ }^{34}$ In chapter 7 , we established that retrospective reports of fatigue in HD patients did not accurately represent and overestimated the actual experienced fatigue severity (i.e. a memoryexperience gap). ${ }^{29}$ Although momentary symptom assessments provide a more 
accurate reflection of symptom experiences in real-life, they do not necessarily have to replace conventional retrospective reports all together. Retrospective and momentary fatigue reports can be used alongside each other and complement each other, as they seem to draw information from different sources (i.e. experiencing self vs. remembering self), which may have a different function (accuracy vs. functionality and guidance of future behaviour). ${ }^{29,35}$ As such, they can be used for different types of outcome. For instance, ESM based self-reports may be preferable for diagnostic decision making or treatment follow-up, as they provide insight in symptom variations and related contextual variables, whereas retrospective symptom assessments may be more relevant when the focus of interest is understanding patients' illness behaviour. $^{36}$

The use of ESM-based symptom reports in clinical practice is currently relatively limited. Most clinical experience with ESM comes from patients with psychopathology, such as psychosis and depression, in which ESM is used as a diagnostic tool to identify risk factors for psychotic or depressive symptoms and reveal symptom patterns. ${ }^{37-41}$ In other medical disciplines, ESM-based symptom reports are gradually gaining ground and become valuable and approved diagnostic instruments. For instance, in neurology (e.g. patients suffering from fatigue after stroke or acquired brain injury ${ }^{42,43}$ ), in gastroenterology (e.g. patients suffering from pain due to irritable bowel syndrome or dyspepsia ${ }^{44-46}$ ), and even in urology (e.g. patients with functional urologic complaints, such as the over active bladder syndrome ${ }^{47}$ ), ESM-based symptom reports are applied to provide insight in symptom development and related contextual factors. In addition to its diagnostic qualities, previous research in depressed and psychotic patients established that ESM may serve as an outcome measure to evaluate the impact of pharmacological treatment on (depressive and psychotic) symptoms, and enables detecting early signs of relapse when tapering off medication. ${ }^{41,48}$ Moreover, ESM data may be used as personalized feedback instrument to support self-insight in symptom development, enhance shared decision making processes, facilitate the ability of self-management and improve compliance. ${ }^{40,49}$ As such, ESM may be used as a therapeutic tool in its own right, complementing medical therapy. For instance, in a recently conducted randomized control trial in depressed outpatients receiving pharmacological treatment and ESM-derived personalized feedback, focusing on positive affect and its context, resulted feasible and led to a significant and clinically relevant decrease of 
depressive symptoms. ${ }^{50}$ Interestingly, in a recent clinical trial in patients suffering from depression or anxiety disorder, ESM was combined with acceptance and commitment psychotherapy, promoting skill building in daily life (ACT-DL). ${ }^{51}$ Despite no statistically significant short term effects on psychological flexibility and quality of life were observed and no long term effects were investigated, results showed that it is feasible to deliver ACT as an mHealth intervention.

Taken together, these findings suggest that ESM has the potential to be a valuable diagnostic instrument, outcome measure for treatment efficacy and even therapeutic tool. Indeed, by providing personalized insights in the relationship between symptoms and environmental variables, ESM paves the way towards personalized interventions, tailored to the needs of the individual. This is especially of interest in conditions, such as fatigue in HD patients, where a one-size fits all treatment may not exist.

\section{Methodological considerations}

Methodological considerations for each study in this thesis have already been addressed in the individual chapters. In this section, some major strengths and limitations that need to be taken into account when interpreting the findings presented in this thesis will be highlighted.

\section{Strengths}

In this thesis, we quantitatively demonstrated the variability in fatigue, and to a larger extent also mood, between and within HD patients. In addition, we were the first to quantitatively establish the development of PDF as an increase in fatigue experience during the haemodialysis treatment. Furthermore, we were the first to implement an $m$ Health ESM procedure to assess fatigue in HD patients and its relationship with psychological and contextual factors in daily life. The amount of ESM data gave us the opportunity to perform time-lagged analyses, which led to new insights in the temporal relationships between fatigue and related psychological and environmental factors. As such, we were able to make preliminary propositions about cause-effect relationships. That is, depressed mood may be secondary to fatigue in the short term and relaxing appears to be a behavioural response to fatigue rather than the other way around. Additionally, time-lagged analyses also provided arguments to 
corroborate the construct of PDF (i.e. undergoing HD treatment, being at the HD unit or with a health care professional at an earlier moment in time predicted significantly higher fatigue at a later time point). Moreover, ESM enabled us to demonstrate that, when administering conventional retrospective fatigue assessment reports in HD patients, a memory-experience gap may occur, resulting in a misrepresentation of the actual experienced fatigue. Similarly, we applied a wearable motion sensor, instead of using subjective retrospective self-reports, to objectively measure HD patients' daily physical activity by means of daily step count. As a result, we prevented recall bias and fake compliance, and found that there was no linear relationship between fatigue and spontaneous daily physical activity in the studied HD population. Developing wearable devices that objectively monitor both physiological and behavioural parameters in 'real-time' is an interesting goal for future research. Further, we were able to test the relationship between PDF and a relatively larger set of inflammatory cytokines compared to previous research studies. Finally, by conducting studies in both Italy and the Netherlands, we were able to add a cross-cultural aspect to this thesis, which adds to the generalizability of the presented findings.

\section{Limitations}

A general and important limitation of the studies presented in this thesis concerns their study design. The first part of this thesis covered studies with cross-sectional and observational study designs. Therefore, assuming any causal relationship between inflammation and fatigue in HD patients remains impossible. The studies addressed in the second and third part of this thesis, due to their longitudinal nature and the ability to perform time-lagged analyses, allowed us to draw some conclusions about the temporal relationships between variables of interest. This may provide information about the causal direction of these relationships. However, establishing temporal precedence (i.e. that the cause occurs before the effect) is a necessary but not sufficient condition for causality. Therefore, advancing our understanding of the causal relationships between the variables studied in this thesis remains a major challenge for future research; a challenge to which this thesis has provided a unique contribution.

An additional limitation of the studies presented in the first and second part of this thesis relates to the relatively small sample sizes (chapter 2 to 5 ). Although the third part of this thesis (chapter 6 and 7) includes multicentre studies, it remains that 
standard dialysis units usually have a limited patient capacity. Future studies in larger samples are warranted. Nevertheless, the studies in part three of this thesis described results that were based on a large set of ESM observations $(n=1777)$ nested within individuals. Indeed, because the main analyses here were conducted at the level of ESM observations and not at the level of the individual, a lack of statistical power was not an issue here.

Moreover, it is worth mentioning that different measurement instruments for both fatigue and depression (i.e. the vitality scale of the 36-item Short Form Health Survey vs. the Fatigue Severity Scale (FSS) and the Beck Depression Inventory-II (BDI) vs. the Hospital Anxiety and Depression Scale (HADS) vs. the Geriatric Depression Scale (GDS), respectively) have been used throughout the conducted studies. Inconsistency between studies concerning the measurement instruments used, may have led to different results and complicate the interpretation and comparison of data. This underlines the need for a standardized and validated measurement instrument for fatigue in HD patients, which may facilitate the comparison of study results from future research.

Another issue when interpreting our data concerns the instruments used to differentiate between depressed and non-depressed HD patients. Although validated to screen for depression, these instruments are symptom-based rating scales which also inquire features of fatigue (e.g. troubles concentrating or making decisions), sleep disorders and even loss of appetite. Due to the overlapping symptomatology of depression, fatigue and symptoms inherent to end-stage kidney disease, which do not necessarily point to the presence of depression, patients may have been misclassified as being depressed. Therefore, future studies may consider assigning patients based on an accurate clinical diagnosis of depression according to the criteria of the Diagnostic and Statistical Manual of Mental Disorders - 5 (DSM-V). ${ }^{52}$

Finally, using mHealth ESM-based symptom assessments may cause some form of patient burden and cannot exclude a potential selection bias as a certain level of cognitive capacity is needed to handle the application. However, future studies could benefit from careful item selection and reduction of questions, based on patients' feedback interviews, to reduce patients' burden. Moreover, it is conceivable that repeatedly inquiring about fatigue and mood may provoke some attention bias. That 
is, patients may be more focused on their symptoms and associated mood, potentially aggravating the perception of their symptom's severity and centrality in daily life. This may be counterbalanced by simultaneous assessments of positive mood states and the promotion of adaptive behaviour (e.g. physical activity).

\section{Clinical implications}

Quality of care in HD patients is still too often determined by the attainment of target parameters of dialysis adequacy and metabolic derangements with the ultimate aim to reduce morbidity and mortality. ${ }^{3}$ However, symptom burden should be given a central place in HD patients' treatment as well. Indeed, shifting the focus from a strictly medical treatment model towards a biopsychosocial treatment model, with an increased interest in patient-reported outcomes, may be beneficial for HD patients. Unfortunately, health care professionals do not always recognize the presence of physical and emotional symptoms in their HD patients, or they may underestimate the severity. ${ }^{53}$ Fatigue may even be perceived as a normal consequence of the illness and treatment burden. ${ }^{53} \mathrm{~A}$ better awareness among professionals is needed and may be achieved by implementing symptom assessments on a regular basis. Indeed, it would be appropriate if professionals extended their care even more to issues that matter most to the patients, such as symptom burden and quality of life.

In this thesis, it was shown that psychological and contextual factors may be equally important to biological factors in explaining variance in fatigue in HD patients. As such, they should be integrated in the standard assessment of fatigue in HD patients. This may be facilitated by implementing electronic ESM procedures, which allow gaining insight in symptom development and related factors. Therefore, the use of electronic ESM procedures should be promoted in routine clinical practice in HD patients. Moreover, ESM provides an excellent opportunity to integrate patients' daily life experiences in a standardized patient-reported outcome measure (PROM) for fatigue in HD patients.

With respect to fatigue management in HD patients, interventions should be tailored to each patients' specific needs, as results of our studies showed that the fatigue severity as well as the contributing factors may differ among patients. It is self-evident 
that any biological factor that may contribute to HD patients' fatigue symptoms (e.g. severe anaemia) needs to be treated primarily. Secondly, individual psychological and contextual factors may become additional treatment targets to alleviate fatigue in HD patients, and mHealth ESM procedures may be an excellent screening and monitoring tool for this.

\section{Directions for future research}

In the previous sections of this chapter, directions for future research to advance our understanding of fatigue in HD patients and develop effective therapies have already been addressed. These included conducting studies in larger samples and intervention trials (e.g. CBT-based therapy). This may help to expand upon the novel findings of this thesis' studies.

In the next part, an important issue concerning the assessment of fatigue in HD patients warranting future research will be discussed. Additionally, some potential targets for future investigation and treatment will be suggested.

In my view, an important next step is the development of a universally accepted and adequate PROM for fatigue in HD patients. Currently used fatigue questionnaires have been originally developed for other patient populations and may not have been tested and validated in the HD population. ${ }^{54}$ Moreover, their recall period usually surpasses 24 hours, risking recall bias as they rely on patients' autobiographical memory. ${ }^{55}$ Further, the questionnaire used to assess fatigue in part III of this thesis was based on a previously used ESM questionnaire conducted for other clinical populations than the HD population (i.e. patients suffering from stroke and schizophrenia). ${ }^{43,56}$ Essentially, A PROM needs to be developed in accordance with the requirements of the U.S. Food and Drug Administration (FDA). ${ }^{57}$ This involves initiating from a conceptual framework, in which the concepts and domains to be measured (e.g. physical and mental fatigue, or general and treatment-related fatigue) are determined. This is usually based on literature and expert opinion. Next, the provisional PROM may be modified in consultation with the target population to ascertain that the PROM reflects the dimensions that are most important to the patients (e.g. the impact of fatigue on their ability to participate in daily life). Indeed, the input of the target 
population is indispensible when developing PROMs. In 2016, the Fatigue Working Group of the International Standardized Outcomes in Nephrology (SONG) initiative took this first step towards the development of a PROM for fatigue in HD patients. ${ }^{58}$ However, results still are awaited and additional examinations, such as assessment of the PROM's psychometric properties, will be required. A final objective may be to combine this PROM for fatigue in HD patients with mHealth ESM technology. Then, it may have the potential to become a valuable and excellent outcome measure for fatigue in HD patients. Moreover, besides the development of a fatigue PROM, a detailed universal characterization of PDF is necessary to improve diagnostic evaluation and management strategies.

Second, this thesis mainly focussed on the link between fatigue in HD patients and depressed mood. However, other psychological and behavioural factors that have been associated with fatigue in HD, such as anxiety, ${ }^{28,59-63}$ sleep disorders or poor sleep quality, ${ }^{60,62,64-66}$ may be interesting avenues for future investigation. Indeed, fatigue, depression, anxiety and poor sleep quality may be interrelated. Therefore, future trials may consider interventions, such as CBT, that focus on patients' underlying depression, anxiety disorder or sleep problem and evaluate whether this improves patients' energy levels and reduces their fatigue experience.

Finally, although we failed to demonstrate that IL-6 may be a common biological mediator for fatigue and depression in HD patients (chapter 2), it remains an interesting avenue for further investigation. After all, in both the general and HD population, fatigue and depression oftentimes co-exist and depression has been associated with inflammation and an altered cortisol metabolism. ${ }^{67}$ Interestingly, other researchers found a significant association between IL-6 levels and depression in a larger cohort of HD patients, while using the BDI, whereas we used the GDS, to assess depression. ${ }^{68,69}$ This once again demonstrates the importance of consistent use of measurement instruments in research. Future studies, in larger sample sizes, diagnosing depression according to the criteria of the DSM-V, may help to clarify whether inflammation plays a role in the development of depression in HD patients. 


\section{Conclusions and closing remark}

Findings of this thesis demonstrated that fatigue in HD patients is a complex phenomenon, changing over time and determined by biological, psychological and contextual factors. On the one hand, we showed that inflammation may be involved in the development and maintenance of a general fatigue experience in HD patients. On the other hand, we showed that psychological and contextual variables may play an equally important role in the development or perpetuation of fatigue in HD patients. Therefore, we believe that the best approach for both diagnosis and management of fatigue in HD patients is achieved by integrating all contributing biological, psychological and social factors. The ESM seems to be a practical and excellent technology to facilitate the identification of fatigue's variability and its relationship with psychological and contextual factors in HD patients' daily life. Given the high prevalence of fatigue and its impact on HD patients' health-related quality of life, additional research into effective therapies is crucial. I hope this thesis may be a source of inspiration for future trials and incites a comprehensive vision that moves beyond a one-sided focus on illness towards a broad consideration of health and wellbeing of individuals with kidney disease. 


\section{References}

1. Riley WT, Rothrock N, Bruce B, et al. Patient-reported outcomes measurement information system (PROMIS) domain names and definitions revisions: further evaluation of content validity in IRTderived item banks. Qual Life Res. 2010;19(9):1311-1321.

2. Weisbord SD, Fried LF, Arnold RM, et al. Prevalence, severity, and importance of physical and emotional symptoms in chronic hemodialysis patients. Journal of the American Society of Nephrology : JASN. 2005;16(8):2487-2494.

3. Davison SN, Jhangri GS. Impact of pain and symptom burden on the health-related quality of life of hemodialysis patients. J Pain Symptom Manage. 2010;39(3):477-485.

4. Urquhart-Secord R, Craig JC, Hemmelgarn B, et al. Patient and Caregiver Priorities for Outcomes in Hemodialysis: An International Nominal Group Technique Study. American journal of kidney diseases : the official journal of the National Kidney Foundation. 2016;68(3):444-454.

5. Dantzer R, Heijnen CJ, Kavelaars A, Laye S, Capuron L. The neuroimmune basis of fatigue. Trends Neurosci. 2014;37(1):39-46.

6. Yao Q, Lindholm B, Stenvinkel P. Inflammation as a cause of malnutrition, atherosclerotic cardiovascular disease, and poor outcome in hemodialysis patients. Hemodialysis international. International Symposium on Home Hemodialysis. 2004;8(2):118-129.

7. Morris G, Berk M, Walder K, Maes M. Central pathways causing fatigue in neuro-inflammatory and autoimmune illnesses. BMC Med. 2015;13:28.

8. Flachenecker P, Bihler I, Weber F, Gottschalk M, Toyka KV, Rieckmann P. Cytokine mRNA expression in patients with multiple sclerosis and fatigue. Multiple sclerosis (Houndmills, Basingstoke, England). 2004;10(2):165-169.

9. Heesen C, Nawrath L, Reich C, Bauer N, Schulz KH, Gold SM. Fatigue in multiple sclerosis: an example of cytokine mediated sickness behaviour? Journal of neurology, neurosurgery, and psychiatry. 2006;77(1):34-39.

10. Moreland LW, Genovese MC, Sato R, Singh A. Effect of etanercept on fatigue in patients with recent or established rheumatoid arthritis. Arthritis and rheumatism. 2006;55(2):287-293.

11. Davis MC, Zautra AJ, Younger J, Motivala SJ, Attrep J, Irwin MR. Chronic stress and regulation of cellular markers of inflammation in rheumatoid arthritis: implications for fatigue. Brain Behav Immun. 2008;22(1):24-32.

12. Saligan LN, Kim HS. A systematic review of the association between immunogenomic markers and cancer-related fatigue. Brain Behav Immun. 2012;26(6):830-848.

13. Schubert C, Hong S, Natarajan L, Mills PJ, Dimsdale JE. The association between fatigue and inflammatory marker levels in cancer patients: a quantitative review. Brain Behav Immun. 2007;21(4):413-427.

14. Singh NP, Bansal R, Thakur A, Kohli R, Bansal RC, Agarwal SK. Effect of membrane composition on cytokine production and clinical symptoms during hemodialysis: a crossover study. Renal failure. 2003;25(3):419-430.

15. Dreisbach AW, Hendrickson T, Beezhold D, Riesenberg LA, Sklar AH. Elevated levels of tumor necrosis factor alpha in postdialysis fatigue. The International journal of artificial organs. 1998;21(2):83-86.

16. Bossola M, Luciani G, Giungi S, Tazza L. Anorexia, fatigue, and plasma interleukin-6 levels in chronic hemodialysis patients. Renal failure. 2010;32(9):1049-1054.

17. Bossola M, Di Stasio E, Giungi S, Rosa F, Tazza L. Fatigue is associated with serum interleukin- 6 levels and symptoms of depression in patients on chronic hemodialysis. I Pain Symptom Manage. 2015;49(3):578-585. 
18. Schmidt ME, Semik J, Habermann N, Wiskemann J, Ulrich CM, Steindorf K. Cancer-related fatigue shows a stable association with diurnal cortisol dysregulation in breast cancer patients. Brain Behav Immun. 2016;52:98-105.

19. Raff $\mathrm{H}$, Trivedi $\mathrm{H}$. Circadian rhythm of salivary cortisol, plasma cortisol, and plasma ACTH in end-stage renal disease. Endocrine connections. 2013;2(1):23-31.

20. Hoving JL, Bartelds GM, Sluiter JK, et al. Perceived work ability, quality of life, and fatigue in patients with rheumatoid arthritis after a 6-month course of TNF inhibitors: prospective intervention study and partial economic evaluation. Scandinavian journal of rheumatology. 2009;38(4):246-250.

21. Minnock P, Kirwan J, Bresnihan B. Fatigue is a reliable, sensitive and unique outcome measure in rheumatoid arthritis. Rheumatology (Oxford, England). 2009;48(12):1533-1536.

22. Keystone E, Burmester GR, Furie R, et al. Improvement in patient-reported outcomes in a rituximab trial in patients with severe rheumatoid arthritis refractory to anti-tumor necrosis factor therapy. Arthritis and rheumatism. 2008;59(6):785-793.

23. Illei GG, Shirota $\mathrm{Y}$, Yarboro $\mathrm{CH}$, et al. Tocilizumab in systemic lupus erythematosus: data on safety, preliminary efficacy, and impact on circulating plasma cells from an open-label phase I dosageescalation study. Arthritis and rheumatism. 2010;62(2):542-552.

24. Norheim KB, Jonsson G, Omdal R. Biological mechanisms of chronic fatigue. Rheumatology (Oxford, England). 2011;50(6):1009-1018.

25. Surawy C, Hackmann A, Hawton K, Sharpe M. Chronic fatigue syndrome: a cognitive approach. Behaviour research and therapy. 1995;33(5):535-544.

26. van Kessel K, Moss-Morris R. Understanding multiple sclerosis fatigue: a synthesis of biological and psychological factors. J Psychosom Res. 2006;61(5):583-585.

27. Skerrett TN, Moss-Morris R. Fatigue and social impairment in multiple sclerosis: the role of patients' cognitive and behavioral responses to their symptoms. J Psychosom Res. 2006;61(5):587-593.

28. Picariello F, Moss-Morris R, Macdougall IC, Chilcot AJ. The role of psychological factors in fatigue among end-stage kidney disease patients: a critical review. Clin Kidney J. 2017;10(1):79-88.

29. Van den Bergh O, Walentynowicz M. Accuracy and bias in retrospective symptom reporting. Current opinion in psychiatry. 2016;29(5):302-308.

30. van den Akker LE, Beckerman H, Collette EH, Eijssen IC, Dekker J, de Groot V. Effectiveness of cognitive behavioral therapy for the treatment of fatigue in patients with multiple sclerosis: $A$ systematic review and meta-analysis. J Psychosom Res. 2016;90:33-42.

31. Kangas $\mathrm{M}$, Bovbjerg DH, Montgomery GH. Cancer-related fatigue: a systematic and meta-analytic review of non-pharmacological therapies for cancer patients. Psychological bulletin. 2008;134(5):700741.

32. Picariello F, Moss-Morris R, Macdougall IC, et al. Cognitive-behavioural therapy (CBT) for renal fatigue (BReF): a feasibility randomised-controlled trial of $C B T$ for the management of fatigue in haemodialysis (HD) patients. BMJ open. 2018;8(3):e020842.

33. Jakubowski KP, Jhamb M, Yabes J, et al. Technology-assisted cognitive-behavioral therapy intervention for end-stage renal disease. Translational behavioral medicine. 2020;10(3):657-663.

34. Shiffman S, Stone AA, Hufford MR. Ecological momentary assessment. Annual review of clinical psychology. 2008;4:1-32.

35. Conway MA, Pleydell-Pearce CW. The construction of autobiographical memories in the self-memory system. Psychological review. 2000;107(2):261-288.

36. Horne R, Chapman SC, Parham R, Freemantle N, Forbes A, Cooper V. Understanding patients' adherence-related beliefs about medicines prescribed for long-term conditions: a meta-analytic review of the Necessity-Concerns Framework. PloS one. 2013;8(12):e80633.

37. Myin-Germeys I, Birchwood M, Kwapil T. From environment to therapy in psychosis: a real-world momentary assessment approach. Schizophrenia bulletin. 2011;37(2):244-247. 
38. Myin-Germeys I, Oorschot M, Collip D, Lataster J, Delespaul P, van Os J. Experience sampling research in psychopathology: opening the black box of daily life. Psychological medicine. 2009;39(9):15331547.

39. Wichers M, Simons CJ, Kramer IM, et al. Momentary assessment technology as a tool to help patients with depression help themselves. Acta psychiatrica Scandinavica. 2011;124(4):262-272.

40. Verhagen SJW, Berben JA, Leue $C$, et al. Demonstrating the reliability of transdiagnostic mHealth Routine Outcome Monitoring in mental health services using experience sampling technology. PloS one. 2017;12(10):e0186294.

41. Wichers M, Groot PC. Critical Slowing Down as a Personalized Early Warning Signal for Depression. Psychotherapy and psychosomatics. 2016;85(2):114-116.

42. Lenaert B, Colombi M, van Heugten $C$, Rasquin S, Kasanova Z, Ponds R. Exploring the feasibility and usability of the experience sampling method to examine the daily lives of patients with acquired brain injury. Neuropsychological rehabilitation. 2019;29(5):754-766.

43. Lenaert B, Neijmeijer M, van Kampen N, van Heugten C, Ponds R. Poststroke Fatigue and Daily Activity Patterns During Outpatient Rehabilitation: An Experience Sampling Method Study. Archives of physical medicine and rehabilitation. 2020;101(6):1001-1008.

44. Mujagic Z, Leue C, Vork L, et al. The Experience Sampling Method--a new digital tool for momentary symptom assessment in IBS: an exploratory study. Neurogastroenterology and motility : the official journal of the European Gastrointestinal Motility Society. 2015;27(9):1295-1302.

45. Vork L, Keszthelyi D, Mujagic Z, et al. Development, content validity, and cross-cultural adaptation of a patient-reported outcome measure for real-time symptom assessment in irritable bowel syndrome. Neurogastroenterology and motility : the official journal of the European Gastrointestinal Motility Society. 2018;30(3).

46. Smeets FGM, Keszthelyi D, Vork L, et al. Development of a real-time patient-reported outcome measure for symptom assessment in patients with functional dyspepsia using the experience sampling method. Neurogastroenterology and motility : the official journal of the European Gastrointestinal Motility Society. 2019;31(2):e13496.

47. Herrewegh A, Vork L, Eurelings $E$, et al. The development of a patient-reported outcome measure for real-time symptom assessment in a population with functional urologic complaints-A focus group study. Neurourology and urodynamics. 2018;37(8):2893-2903.

48. Bak M, Drukker M, Hasmi L, van Os J. An $n=1$ Clinical Network Analysis of Symptoms and Treatment in Psychosis. PloS one. 2016;11(9):e0162811.

49. van Os J, Verhagen S, Marsman A, et al. The experience sampling method as an mHealth tool to support self-monitoring, self-insight, and personalized health care in clinical practice. Depression and anxiety. 2017;34(6):481-493.

50. Kramer I, Simons CJ, Hartmann JA, et al. A therapeutic application of the experience sampling method in the treatment of depression: a randomized controlled trial. World psychiatry : official journal of the World Psychiatric Association (WPA). 2014;13(1):68-77.

51. Batink T, Bakker J, Vaessen T, et al. Acceptance and Commitment Therapy in Daily Life Training: A Feasibility Study of an mHealth Intervention. JMIR mHealth and uHealth. 2016;4(3):e103.

52. Association AP. Diagnostic and statistical manual of mental disorders (5th ed.). Washington DC; 2013.

53. Weisbord SD, Fried LF, Mor MK, et al. Renal provider recognition of symptoms in patients on maintenance hemodialysis. Clinical journal of the American Society of Nephrology : CJASN. 2007;2(5):960-967.

54. Ju A, Unruh ML, Davison SN, et al. Patient-Reported Outcome Measures for Fatigue in Patients on Hemodialysis: A Systematic Review. American journal of kidney diseases : the official journal of the National Kidney Foundation. 2018;71(3):327-343. 
55. Whitehead L. The measurement of fatigue in chronic illness: a systematic review of unidimensional and multidimensional fatigue measures. J Pain Symptom Manage. 2009;37(1):107-128.

56. Oorschot $M$, Lataster $T$, Thewissen V, et al. Emotional experience in negative symptoms of schizophrenia--no evidence for a generalized hedonic deficit. Schizophrenia bulletin. 2013;39(1): 217-225.

57. Guidance for industry: patient-reported outcome measures: use in medical product development to support labeling claims: draft guidance. Health and quality of life outcomes. 2006;4:79.

58. Ju A, Unruh M, Davison S, et al. Establishing a Core Outcome Measure for Fatigue in Patients on Hemodialysis: A Standardized Outcomes in Nephrology-Hemodialysis (SONG-HD) Consensus Workshop Report. American journal of kidney diseases : the official journal of the National Kidney Foundation. 2018;72(1):104-112.

59. Bossola M, Ciciarelli C, Di Stasio E, et al. Correlates of symptoms of depression and anxiety in chronic hemodialysis patients. General hospital psychiatry. 2010;32(2):125-131.

60. Bossola M, Luciani G, Tazza L. Fatigue and its correlates in chronic hemodialysis patients. Blood purification. 2009;28(3):245-252.

61. Chilcot J, Moss-Morris R, Artom M, et al. Psychosocial and Clinical Correlates of Fatigue in Haemodialysis Patients: the Importance of Patients' Illness Cognitions and Behaviours. International journal of behavioral medicine. 2016;23(3):271-281.

62. McCann K, Boore JR. Fatigue in persons with renal failure who require maintenance haemodialysis. Journal of advanced nursing. 2000;32(5):1132-1142.

63. Wang SY, Zang XY, Liu JD, Gao M, Cheng M, Zhao Y. Psychometric properties of the Functional Assessment of Chronic Illness Therapy-Fatigue (FACIT-Fatigue) in Chinese patients receiving maintenance dialysis. J Pain Symptom Manage. 2015;49(1):135-143.

64. Jhamb M, Pike F, Ramer S, et al. Impact of fatigue on outcomes in the hemodialysis (HEMO) study. American journal of nephrology. 2011;33(6):515-523.

65. Jhamb M, Liang K, Yabes J, et al. Prevalence and correlates of fatigue in chronic kidney disease and end-stage renal disease: are sleep disorders a key to understanding fatigue? American journal of nephrology. 2013;38(6):489-495.

66. Joshwa B, Khakha DC, Mahajan S. Fatigue and depression and sleep problems among hemodialysis patients in a tertiary care center. Saudi journal of kidney diseases and transplantation : an official publication of the Saudi Center for Organ Transplantation, Saudi Arabia. 2012;23(4):729-735.

67. Howren MB, Lamkin DM, Suls J. Associations of depression with C-reactive protein, IL-1, and IL-6: a meta-analysis. Psychosomatic medicine. 2009;71(2):171-186.

68. Taraz M, Khatami MR, Gharekhani A, Abdollahi A, Khalili H, Dashti-Khavidaki S. Relationship between a pro- and anti-inflammatory cytokine imbalance and depression in haemodialysis patients. European cytokine network. 2012;23(4):179-186.

69. Taraz M, Taraz S, Dashti-Khavidaki S. Association between depression and inflammatory/antiinflammatory cytokines in chronic kidney disease and end-stage renal disease patients: a review of literature. Hemodialysis international. International Symposium on Home Hemodialysis. 2015;19(1):11-22. 



\section{Addendum}

Summary 
Addendum 


\section{Summary}

Chronic kidney disease is characterized by inadequate renal functioning and may progress into end-stage kidney disease, requiring renal replacement therapy to sustain life. Haemodialysis (HD) therapy is the most frequently applied modality of renal replacement therapy and filters the blood for waste products and excessive fluid via an artificial extracorporeal blood circuit. In the next decades, the prevalence of end-stage kidney disease is expected to rise significantly due to the global expansion of diabetes and cardiovascular diseases. Although HD treatment may be a lifeextending therapy, the health-related quality of life (HrQoL) of patients receiving chronic HD treatment is poor due to discomforting symptoms. One of the most common symptoms is fatigue, affecting up to $90 \%$ of HD patients. Therefore, fatigue is a critical important outcome for the HD population. However, no effective interventions for managing and improving fatigue in HD patients have been developed yet. This may be complicated by the lack of knowledge about its underlying aetiology and the absence of an appropriate measurement instrument that takes into account the multifactorial nature of fatigue in HD patients. This thesis aimed to advance our understanding of the development and maintenance of fatigue in HD patients and to investigate whether the use of mobile health (mHealth) technology may help to unravel the multifactorial nature of fatigue in HD patients.

Chapter 1 provides a general introduction to the studies in this thesis. The factors studied so far that may be related to fatigue in HD patients, such as depressed mood, are summarized and remaining knowledge gaps are identified. Moreover, limitations of conventional fatigue measurement instruments are discussed and the Experience Sampling Method (ESM), a structured diary method to capture symptoms, experience, and behaviour in daily life, is introduced as a potential suitable measurement technology to advance our understanding of fatigue in HD patients.

Part I relates to the potential biological factors that may be involved in the development of fatigue in HD patients and examines the hypothesis that fatigue in HD patients may be triggered by inflammation. Chapter 2 presents data of a crosssectional observational study in 59 HD patients, investigating the interrelationships between general fatigue, inflammation - by means of serum endotoxin and serum interleukin-6 levels - and depressed mood. Findings showed that HD patients' general 
fatigue experience is positively associated with their level of inflammation and depressed mood. Moreover, a multiple regression analysis revealed that $31 \%$ of the variance in HD patients' general fatigue is explained by inflammation and depressed mood. Chapter 3 builds further on the inflammation-induced fatigue hypothesis and investigates in a cross-sectional observational study $(\mathrm{N}=45)$ whether fatigue occurring immediately after the HD session (i.e. postdialysis fatigue (PDF)) may be related to changes in pro- or anti-inflammatory cytokine levels, potentially resulting from the HD procedure. Remarkably, we found no evidence for an association between PDF and alterations in inflammatory cytokine levels. Together, the findings from chapter 2 and 3 suggest that inflammation may be partially involved in the development of general fatigue in HD patients, but not in PDF. Moreover, these studies indicate that other factors besides biological variables may determine fatigue in HD patients.

Part II focuses on the variability in fatigue in HD patients and assesses to what extent psychological and contextual factors may be related to fatigue experience in HD patients. In chapter 4, we report on a longitudinal observational study in 51 HD patients, investigating the diurnal changes in fatigue experience and the relationship with the HD treatment, patients' mood and the hospital environment. It is shown that fatigue in HD patients varies over time and increases after the HD treatment. Moreover, HD patients' diurnal fatigue patterns differed as a function of mood, with depressed individuals experiencing a more chronic and severe fatigue pattern, whereas non-depressed individuals show a more fluctuating and less severe fatigue pattern. Interestingly, we also observed that fatigue in HD patients may - at least partially - be explained as a classically conditioned response to the hospital environment. In chapter 5, we present results from a longitudinal observational study in $37 \mathrm{HD}$ patients, examining the relationship between spontaneous daily physical activity, objectively measured by means of a wearable motion sensor recording daily step count, and fatigue and depressed mood, respectively. Findings showed that HD patients' spontaneous daily physical activity was not significantly associated with their fatigue experience or with their mood. Altogether, part II corroborates that psychological and contextual variables may contribute to fatigue in HD patients above and beyond biological factors.

Part III covers the implementation of an mHealth ESM procedure to unravel the multifactorial nature of fatigue in HD patients and assesses whether discrepancies 
may arise between 'real-time' and retrospective fatigue reports. In chapter 6, we conducted a longitudinal observational study $(\mathrm{N}=40)$, using an mHealth ESM application to gain detailed information about the relationship between fatigue and psychological (e.g. depressive feelings), social (e.g. social company) and contextual (e.g. daily activity or location) factors in HD patients' daily life. Findings reaffirm that fatigue varies between and within HD patients, and that fatigue relates to situational factors in individuals' daily life. Time-lagged analyses allowed to make preliminary propositions about the cause-effect relationship between fatigue and psychological (e.g. feeling down seems to be secondary to fatigue in the short term) and contextual factors (e.g. relaxing seems a behavioural response to fatigue rather than the other way around), respectively. Chapter 7 presents data of the same study, comparing retrospective fatigue paper reports with 'real-time' fatigue scores, assessed with the mHealth ESM application. We observed that retrospective fatigue evaluation may misrepresent actual symptom experience, corroborating the hypothesis of a so-called 'memory-experience gap' for fatigue experience in HD patients. These findings may suggest that 'real-time' and retrospective symptom assessments reflect different types of information (e.g. accuracy vs. guidance of future behaviour, respectively) and may be used for different types of outcome (e.g. diagnostic decision making vs. understanding illness behaviours, respectively). Taken together, mHealth ESM procedures allow gaining detailed and personalized insight in 'real-time' fatigue experience. Moreover, it allows linking fatigue experiences to situational and contextual factors in patients' daily life, paving the way towards personalized interventions.

Chapter 8 provides a general discussion, covering a recapitulation and interpretation of the main findings of this thesis' studies, methodological considerations, clinical implications and directions for future research. The studies described in this thesis showed that fatigue in HD patients changes between and within individuals, and is determined by biological, psychological and contextual factors. As such, approaching fatigue in HD patients according to a biopsychosocial model, integrating all potential contributing factors, is the best way forward for both diagnosis and management. $m$ Health ESM is an excellent method to assess fatigue in HD patients' daily life, taking into account its multifactorial nature, and has the potential to become a valuable outcome measure for fatigue in HD patients. With respect to routine clinical practice, more awareness for HD patients' symptom burden, their HrQoL, and the role of other 
than biological factors contributing to the development and perpetuation of fatigue is warranted. The management of fatigue in HD patients should be tailored to the needs of the individual and target both biological and non-biological determinants. Future research, creating a universally accepted patient-reported outcome measure (PROM) for fatigue in HD patients and developing effective therapies in order to improve HD patients' HrQoL, is needed. 

Samenvatting 
Addendum 


\section{Samenvatting}

Chronisch nierfalen wordt gekenmerkt door een insufficiënte nierfunctie en kan uiteindelijk leiden tot eindstadium nierfalen. Op dat moment is niervervangende therapie noodzakelijk om in leven te blijven. De meest frequent toegepaste vorm van niervervangende therapie is hemodialyse (HD). Hierbij wordt het bloed van afvalstoffen gezuiverd en wordt overtollig lichaamsvocht verwijderd via een kunstmatig extracorporeel bloedcircuit. De prevalentie van eindstadium nierfalen zal naar verwachting in de komende jaren alleen maar toenemen als gevolg van de wereldwijde expansie van diabetes en hart- en vaatziekten. Hoewel behandeling met HD levensverlengend kan zijn, is de kwaliteit van leven van HD patiënten ondermaats. Dit is toe te schrijven aan meerdere hinderlijke symptomen die deze patiënten ervaren. Eén van de meest gerapporteerde klachten is vermoeidheid. Tot $90 \%$ van de HD patiënten heeft last van vermoeidheid. Er zijn echter nog steeds geen effectieve therapieën beschikbaar om vermoeidheid bij HD patiënten aan te pakken of te verbeteren. De ontwikkeling van nieuwe behandelingen wordt bemoeilijkt door een gebrek aan kennis over de onderliggende oorzaken en het gebrek aan een geschikt meetinstrument dat rekening houdt met de multifactoriële aard van vermoeidheid bij HD patiënten. Dit proefschrift tracht onze kennis omtrent de ontwikkeling en instandhouding van vermoeidheid bij HD patiënten te bevorderen én te onderzoeken of het gebruik van mobiele gezondheidstechnologie (mHealth) kan helpen om de multifactoriële aard van vermoeidheid bij HD patiënten te ontrafelen.

Hoofdstuk 1 bevat een algemene inleiding tot de onderzoeken in dit proefschrift. Ten eerste worden de tot dusver bestudeerde factoren besproken die mogelijk verband houden met vermoeidheid bij HD patiënten, waaronder depressieve stemming, en worden resterende hiaten in de kennis omtrent vermoeidheid bij HD patiënten aangekaart. Vervolgens worden de beperkingen van conventionele meetinstrumenten voor vermoeidheid behandeld. Tot slot wordt de Experience Sampling Method (ESM), een gestructureerde dagboekmethode om symptomen, ervaringen en gedragingen in het dagelijks leven van patiënten in kaart te brengen, geïntroduceerd als een mogelijk geschikte meettechnologie om onze kennis omtrent vermoeidheid bij HD patiënten te bevorderen. 
Het eerste deel van dit proefschrift behandelt biologische factoren die mogelijk betrokken kunnen zijn bij de ontwikkeling van vermoeidheid bij HD patiënten. De hypothese dat vermoeidheid bij HD patiënten wordt uitgelokt door inflammatie (ontsteking) wordt bestudeerd. Hoofdstuk 2 toont bevindingen van een crosssectionele observationele studie bij 59 HD patiënten, waarin de onderlinge relaties tussen algemene vermoeidheid, inflammatie - aan de hand van serum endotoxine en serum interleukine- 6 waarden - en depressieve stemming werden onderzocht. De resultaten laten zien dat algemene vermoeidheid bij HD patiënten positief geassocieerd is met zowel inflammatie als depressieve stemming. Een meervoudige regressieanalyse toont bovendien aan dat $31 \%$ van de variantie in algemene vermoeidheid bij HD patiënten wordt verklaard door inflammatie en depressieve stemming. In Hoofdstuk 3 wordt er verder gebouwd op deze inflammatiegeïnduceerde vermoeidheidshypothese. In een cross-sectionele observationele studie $(\mathrm{N}=45)$ onderzochten we of vermoeidheid die meteen na de HD-sessie optreedt (postdialyse vermoeidheid of PDF genoemd) mogelijk verband houdt met veranderingen in serumwaarden van pro- of anti-inflammatoire cytokines als gevolg van de HD-procedure. Opmerkelijk genoeg vonden we geen aanwijzingen voor een verband tussen PDF en veranderingen in inflammatoire cytokinewaarden. Samenvattend, de bevindingen uit hoofdstuk 2 en 3 suggereren dat inflammatie gedeeltelijk betrokken kan zijn bij de ontwikkeling van algemene vermoeidheid bij HD patiënten, maar niet bij PDF. Deze onderzoeken tonen bovendien aan dat naast biologische variabelen ook andere factoren vermoeidheid bij HD patiënten kunnen bepalen.

In het tweede deel van dit proefschrift ligt de focus op de variabiliteit in vermoeidheid bij HD patiënten en onderzoeken we in hoeverre psychologische en contextuele factoren gerelateerd zijn aan vermoeidheidsbeleving bij deze patiënten. Hoofdstuk 4 beschrijft een longitudinale observationele studie bij 51 HD patiënten, bij wie we de dagelijkse schommelingen in vermoeidheid onderzochten. We keken hierbij voornamelijk naar de rol van de HD behandeling en de aanwezigheid van een depressieve stemming. De resultaten tonen dat vermoeidheid bij HD patiënten varieert in de tijd en toeneemt na de HD behandeling. Bovendien verschilt het patroon van vermoeidheid bij HD patiënten doorheen de dag in functie van hun stemming. Depressieve personen vertonen een meer chronisch en ernstig vermoeidheidspatroon, terwijl niet-depressieve personen een meer fluctuerend en 
minder ernstig vermoeidheidspatroon vertonen. Opvallend genoeg vonden we ook aanwijzingen dat vermoeidheid bij HD patiënten - althans gedeeltelijk - kan worden verklaard als een klassiek geconditioneerde respons op de ziekenhuisomgeving. In hoofdstuk 5 presenteren we resultaten van een longitudinale observationele studie bij 37 HD patiënten, bij wie de relatie tussen dagelijkse spontane fysieke activiteit en respectievelijk vermoeidheid en depressieve stemming werd onderzocht. Spontane fysieke activiteit werd in deze studie objectief gemeten met behulp van een draagbare bewegingssensor die het dagelijks aantal stappen registreerde. De bevindingen tonen aan dat dagelijkse spontane fysieke activiteit van HD patiënten niet significant geassocieerd is met hun vermoeidheidsbeleving of met hun stemming. Samenvattend, het tweede deel van dit proefschrift bevestigt dat psychologische en contextuele variabelen kunnen bijdragen aan vermoeidheid bij HD patiënten bovenop de biologische factoren.

Het derde deel van dit proefschrift behandelt de implementatie van een mobielegezondheid-ESM (mHealth ESM) methode om de multifactoriële aard van vermoeidheid bij HD patiënten te ontrafelen. Eveneens bestuderen we of er discrepanties bestaan tussen actuele ('hier-en-nu') en retrospectieve vermoeidheidsbeoordelingen. In hoofdstuk 6 werd een longitudinale observationele studie $(\mathrm{N}=40)$ uitgevoerd, waarbij een mHealth ESM applicatie gebruikt werd om gedetailleerde informatie te verkrijgen over de relatie tussen vermoeidheid en psychologische (bijv. depressieve gevoelens), sociale (bijv. sociaal gezelschap) en contextuele (bijv. dagelijkse activiteit of locatie) factoren in het dagelijks leven van HD patiënten. Bevindingen bevestigen dat de mate van vermoeidheid niet alleen varieert tussen individuen maar ook binnen eenzelfde individu, en dat die variatie verband houdt met factoren in het dagelijks leven van individuen. Met behulp van time-lagged analyses konden we inzicht krijgen in de temporele relaties tussen vermoeidheid en psychologische factoren (bijv. neerslachtigheid lijkt secundair te zijn aan vermoeidheid op korte termijn) en contextuele factoren (bijv. rusten lijkt eerder een gedragsreactie op vermoeidheid dan omgekeerd). Inzicht in dergelijke relaties kunnen een belangrijke stap vormen in het begrijpen van de oorzaken en gevolgen van vermoeidheid in het dagelijks leven. Hoofdstuk 7 presenteert bevindingen van hetzelfde onderzoek. HD patiënten verzamelden eerst een week lang actuele ('hieren-nu') vermoeidheidscores in hun dagelijks leven via de mHealth ESM applicatie op hun smartphone. Nadien werden deze actuele vermoeidheidscores vergeleken met 
patiënten hun retrospectieve inschatting over de ervaren vermoeidheid tijdens diezelfde week aan de hand van een papieren vragenlijst. We stelden vast dat de retrospectieve inschatting hoger lag dan het gemiddelde van alle 'hier-en-nu' vermoeidheidsscores. Dit kan betekenen dat terugkijken in de tijd een vertekend beeld van de werkelijke symptoomervaring oplevert. Deze bevindingen suggereren mogelijk dat actuele en retrospectieve symptoombeoordelingen verschillende soorten informatie weerspiegelen, elk met een eigen functie (bijv. nauwkeurigheid versus sturen van toekomstig gedrag), en kunnen gebruikt worden voor verschillende soorten uitkomsten (bijv. diagnostische besluitvorming versus begrip van ziektegedrag). Samengevat, mHealth ESM methodes maken het mogelijk om een gedetailleerd en persoonlijk inzicht te krijgen in actuele vermoeidheidsbeleving. Bovendien laten ze toe om vermoeidheidsbeleving te koppelen aan situationele en contextuele factoren in het dagelijks leven van patiënten. Dit effent het pad voor meer gepersonaliseerde interventies.

Hoofdstuk 8 omvat een algemene bespreking met een samenvatting en interpretatie van de belangrijkste bevindingen van de studies uit dit proefschrift. Eveneens maak ik methodologische overwegingen, bespreek ik klinische implicaties en maak ik suggesties voor toekomstig onderzoek. De studies beschreven in dit proefschrift tonen aan dat vermoeidheid bij HD patiënten samenhangt met biologische, psychologische en omgevingsfactoren. Daarom is het benaderen van vermoeidheid bij HD patiënten volgens een biopsychosociaal model, waarin alle potentieel bijdragende factoren worden geïntegreerd, de beste weg voorwaarts voor zowel diagnose als behandeling. $m$ Health ESM is een uitstekende methode om vermoeidheid in het dagelijks leven van HD patiënten te beoordelen, omdat het rekening houdt met de multifactoriële aard ervan. Vermoeidheid meten aan de hand van ESM heeft dan ook het potentieel om een waardevolle uitkomstmaat te worden voor vermoeidheid bij HD patiënten. Met betrekking tot de routinematige klinische praktijk is meer aandacht voor de symptoombelasting en levenskwaliteit van HD patiënten noodzakelijk. Daarnaast is het belangrijk om rekening te houden met andere dan biologische factoren die bijdragen aan de ontwikkeling en instandhouding van vermoeidheid. De behandeling van vermoeidheid bij HD patiënten moet afgestemd worden op de behoeften van het individu en zich richten op zowel biologische als niet-biologische determinanten. Een bereikbaar doel voor toekomstig onderzoek ligt in de ontwikkeling van een internationaal aanvaarde patiënt-gerapporteerde uitkomstmaat (patient-reported 
outcome measure of PROM) voor vermoeidheid bij HD patiënten. Ook de ontwikkeling van effectieve behandelingen voor vermoeidheid die HD patiënten meer levenskwaliteit bieden, horen hoog in de onderzoeksagenda te staan. 

Sintesi 
Addendum 


\section{Sintesi}

La malattia renale cronica è caratterizzata da un funzionamento renale inadeguato e può progredire in una malattia renale allo stadio terminale, richiedendo una terapia sostitutiva renale per permettere la vita. La terapia emodialitica (HD) è la modalità più frequentemente applicata nella terapia sostitutiva renale e depura il sangue dalle tossine uremiche ed elimina i fluidi eccessivi attraverso un circuito sanguigno extracorporeo artificiale. Nei prossimi decenni, si prevede che la prevalenza della malattia renale allo stadio terminale aumenterà in modo significativo a causa della diffusione globale del diabete e delle malattie cardiovascolari. Sebbene il trattamento con l'emodialisi costituisca una terapia che prolunga la vita, la qualità della vita dei pazienti che ricevono un trattamento cronico di HD è scarsa a causa di sintomi frequenti e debilitanti. Uno dei sintomi più comuni è l'astenia, che è presente fino al $90 \%$ dei pazienti in HD. Pertanto, l'astenia è un esito importante e critico per la popolazione emodialitica. Tuttavia non sono stati ancora sviluppati interventi efficaci per la gestione e il miglioramento dell'astenia nei pazienti emodialitici. Ciò può essere complicato dalla mancanza di conoscenza della sua eziologia sottostante e dall'assenza di uno strumento di misurazione appropriato che tenga conto della natura multifattoriale dell'astenia nei pazienti in HD.

Scopo della presente tesi è stato definire le caratteristiche dell'astenia nei pazienti HD, e i suoi possibili meccanismi patogenetici e indagare se l'uso della tecnologia mobile health (mHealth) possa aiutare a svelare la natura multifattoriale dell'astenia nei pazienti emodialitici.

Il capitolo 1 fornisce un'introduzione generale agli studi di questa tesi. I fattori studiati finora che possono essere correlati all'astenia nei pazienti in HD, come l'umore depresso, vengono riassunti e vengono identificate le lacune di conoscenza rimanenti. Inoltre, vengono discussi i limiti degli strumenti di misurazione dell'astenia convenzionali e viene introdotto il metodo di campionamento dell'esperienza (Experience Sampling Method, ESM), un metodo di diario strutturato per catturare sintomi, esperienza e comportamento nella vita quotidiana, come potenziale tecnologia di misurazione adatta per far progredire la nostra comprensione dell'astenia in pazienti in HD. 
La parte I si riferisce ai potenziali fattori biologici che possono essere coinvolti nello sviluppo dell'astenia nei pazienti in HD ed esamina l'ipotesi che l'astenia nei pazienti in HD possa essere indotta da uno stato di infiammazione cronica. Il capitolo 2 presenta i dati di uno studio osservazionale trasversale su 59 pazienti in HD, indagando le interrelazioni tra astenia generale, infiammazione - mediante endotossina sierica e livelli di interleuchina-6 sierica - e umore depresso. I risultati hanno mostrato che l'astenia generale dei pazienti in HD è positivamente associata allo stato di infiammazione e ai sintomi di depressione. Inoltre, un'analisi di regressione multipla ha rivelato che il $31 \%$ della varianza nell'astenia generale dei pazienti in HD è "spiegabile" dallo stato di infiammazione cronica e dai sintomi di depressione. Il capitolo 3 riporta i risultati di uno studio osservazionale trasversale $(\mathrm{N}=45)$ che ha valutato se l'astenia che compare immediatamente dopo la sessione di $H D$, ovvero astenia post-dialisi (Postdialysis fatigue, PDF), possa essere correlata a variazioni dei livelli di citochine pro- o anti-infiammatorie, potenzialmente derivanti dalla procedura emodialitica. Sorprendentemente, lo studio ha dimostrato che non esiste una significativa correlazione tra PDF e variazione intra-dialitica delle citochine pro- e anti-infiammatorie.

La parte II si concentra sulla variabilità dell'astenia nei pazienti in HD e valuta in che misura i fattori psicologici e contestuali possono essere correlati all'esperienza dell'astenia nei pazienti in HD. Nel capitolo 4 riportiamo uno studio osservazionale longitudinale su 51 pazienti in HD che ha definito le variazioni giornaliere dei livelli di astenia e la loro relazione con il trattamento emodialitico, i sintomi di depressione, e l'ambiente ospedaliero. Lo studio mostra che l'astenia nei pazienti in HD varia nel tempo e aumenta dopo il trattamento emodialitico. Inoltre, il decorso giornaliero dell'astenia differisce in funzione dei sintomi di depressione. Infatti, nei pazienti con sintomi di depressione più severi, i livelli di astenia erano più elevati e costanti nel tempo, mentre nei pazienti con sintomi di depressione lievi o assenti, i livelli di astenia erano bassi prima dell'inizio del trattamento emodialitico e poi aumentavano progressivamente nel tempo. Inoltre, lo studio ha dimostrato che l'astenia nei pazienti in HD può - almeno parzialmente - essere riconducibile ad una risposta "classicamente condizionata" all'ambiente ospedaliero. Nel capitolo 5 presentiamo i risultati di uno studio osservazionale longitudinale su 37 pazienti in HD, esaminando la relazione tra l'attività fisica quotidiana spontanea, misurata oggettivamente per mezzo di un sensore di movimento indossabile che registra il conteggio dei passi giornalieri, e 
rispettivamente l'astenia e i sintomi di depressione. I risultati hanno mostrato che l'attività fisica quotidiana spontanea dei pazienti in HD non era significativamente associata alla loro esperienza d'astenia o al loro umore. Complessivamente, la parte II evidenzia che le variabili psicologiche e contestuali possono contribuire all'astenia nei pazienti in HD in maggior misura di e indipendentemente dai fattori biologici.

La Parte III riguarda l'implementazione di una procedura mHealth ESM che ha lo scopo di svelare la natura multifattoriale dell'astenia nei pazienti in HD e elabora sulle possibilità di discrepanze tra i rapporti 'in tempo reale' e quelli retrospettivi sull'astenia. Nel capitolo 6 sono riportati i risultati di uno studio osservazionale longitudinale $(\mathrm{N}=40)$ che, utilizzando un'applicazione mHealth ESM, ha valutato la relazione tra astenia e fattori psicologici (es. sintomi di depressione), sociali (es. compagnia sociale) e contestuali (es. attività quotidiana o posizione) nella vita quotidiana dei pazienti in HD. I risultati confermano che l'astenia varia tra e all'interno dei pazienti in HD e che l'astenia è correlata a fattori situazionali nella vita quotidiana degli individui. Le analisi temporali hanno permesso di formulare proposizioni preliminari sulla relazione causa-effetto tra astenia e fattori psicologici (ad es. sentirsi giù sembra essere secondario all'astenia nel breve termine) e fattori contestuali (ad es. rilassarsi sembra una risposta comportamentale all'astenia piuttosto che il contrario), rispettivamente. II capitolo 7 presenta dati dello stesso studio, confrontando i rapporti retrospettivi dell'astenia in cartaceo con i punteggi dell'astenia 'in tempo reale', valutati con l'applicazione mHealth ESM. Abbiamo osservato che la valutazione retrospettiva dell'astenia può travisare l'effettiva esperienza dei sintomi, corroborando l'ipotesi di un cosiddetto "gap di esperienza di memoria" per l'esperienza d'astenia nei pazienti in HD. Questi risultati possono suggerire che le valutazioni dei sintomi 'in tempo reale' e retrospettive riflettono diversi tipi di informazioni (ad es. accuratezza vs guida del comportamento futuro, rispettivamente) e possono essere utilizzate per diversi tipi di risultati (ad esempio, il processo decisionale diagnostico e la comprensione dei comportamenti della malattia, rispettivamente). Nel loro insieme, le procedure $m$ Health ESM consentono di ottenere informazioni dettagliate e personalizzate sull'esperienza d'astenia 'in tempo reale'. Inoltre, consentono di collegare le esperienze d'astenia a fattori situazionali e contestuali nella vita quotidiana dei pazienti, aprendo la strada a interventi personalizzati. 
Il capitolo 8 fornisce una discussione generale, offrendo una ricapitolazione e interpretazione dei principali risultati degli studi di questa tesi, considerazioni metodologiche, implicazioni cliniche e indicazioni per la ricerca futura. Gli studi descritti in questa tesi hanno dimostrato che l'astenia nei pazienti in HD cambia tra e all'interno degli individui ed è determinato da fattori biologici, psicologici e contestuali. Di conseguenza, approcciare l'astenia nei pazienti in HD secondo un modello biopsicosociale, integrando tutti i potenziali fattori che lo causano è la migliore via da seguire, sia per la diagnosi che per la gestione. mHealth ESM è un metodo eccellente per valutare l'astenia nella vita quotidiana dei pazienti $H D$, capace di tener conto della sua natura multifattoriale, e ha il potenziale per diventare una preziosa misura di esito per l'astenia nei pazienti HD.

Per quanto riguarda la pratica clinica, è necessaria una maggiore consapevolezza del carico di sintomi dei pazienti in HD, la loro qualità della vita e il ruolo di fattori diversi da quelli biologici che contribuiscono allo sviluppo e alla cronicizzazione dell'astenia. La gestione dell'astenia nei pazienti in HD dovrebbe essere adattata alle esigenze dell'individuo e mirare a determinanti sia biologiche che non biologiche. Sono necessarie ulteriori ricerche, che crei una misura di esito riportato dai pazienti (patient-reported outcome measure, PROM) universalmente accettata per l'astenia nei pazienti in HD e lo sviluppo di terapie efficaci per migliorare la qualità della vita dei pazienti in HD. 

Impact paragraph 
Addendum 


\section{Impact paragraph}

In this part of the thesis, I will discuss the scientific impact of our research, its relevance for specific target groups and society, and its implementation and innovation. Finally, the dissemination of our knowledge is addressed.

By 2030 chronic kidney disease will be among the 15 leading causes of death. ${ }^{1}$ Haemodialysis (HD) therapy is the most common form of renal replacement therapy for end-stage kidney disease ${ }^{2}$ with an estimated prevalence of 465 and 1437 per million population in Europe and the U.S., respectively. ${ }^{2-4}$ Up to $90 \%$ of HD patients report fatigue as a symptom ${ }^{5}$. However, no effective treatment for fatigue in HD patients is available yet. In order to facilitate the development of effective therapies, this thesis' studies focussed on advancing our understanding about the underlying aetiology of fatigue in HD patients and investigated whether a mobile Health (mHealth) Experience Sampling Methodology (ESM) may be a suitable measurement method to unravel the multifactorial nature of fatigue in HD patients.

\section{Scientific impact}

In this thesis, we showed that fatigue in HD patients may be accounted for by biological factors (e.g. inflammation) as well as psychological (e.g. depressed mood) and contextual (e.g. daily activity, social company, location or sleep quality) variables. Moreover, we demonstrated that fatigue in HD patients varies between and within individuals and that this variability is related to psychological and contextual factors. Therefore, the scientific impact of our research is that fatigue in HD patients should be regarded from a biopsychosocial perspective, in which physiological, psychological and contextual factors may all contribute to the development and maintenance of fatigue in HD patients. Consequently, the focus should shift from a strictly medical treatment model for fatigue in HD patients towards a model integrating psychological and contextual factors. In addition, we demonstrated that using an mHealth ESM technology to assess fatigue in HD patients provides detailed information about the variability in fatigue and allows identifying psychological and contextual factors in HD patients' daily life that contribute to that variability. Moreover, we found that retrospective fatigue assessments by means of conventional fatigue reports may misrepresent real-time symptom experiences measured with mHealth ESM. Therefore, our research demonstrates that mHealth ESM may be a perfect 
measurement method to assess fatigue in HD patients as it enables to gain detailed and personalized information about symptom severity and contributing factors in patients' daily life. As such, ESM offers entry points for interventions other than biological treatment and paves the way towards personalized interventions.

\section{Relevance for HD patients, caregivers, health care professionals and society}

Fatigue is one of HD patients' most prioritized outcomes ${ }^{6}$ as it greatly impacts their health-related quality of life. ${ }^{7}$ Indeed, fatigue may restrict HD patients' participation in all aspects of life. For instance, fatigue in HD patients may lead to sick leave or the loss of ability to work, and may cause withdrawal from hobbies and social contacts. ${ }^{8}$ Moreover, due to fatigue HD patients may be limited in taking up their role in the family and may fail to meet the expectations of other family members regarding family responsibilities, leading to misunderstanding and conflicts $^{8}$. Given that HD treatment is usually received for many years, symptom relief merits equal attention in the management of HD patients. Therefore, research into the development and maintenance of fatigue in HD patients and into effective strategies to alleviate this debilitating symptom is relevant for patients as well as caregivers, health care professionals and society. A better understanding of the factors that contribute to fatigue, including psychological, social or contextual variables, may make fatigue more comprehensible and less vague for HD patients. Psychological counselling may then help patients to cope better with their fatigue and its consequences in daily life. A better understanding of fatigue in HD patients may also increase other people's understanding of HD patients' condition, including caregivers. Furthermore, it may increase health care professionals' awareness for fatigue symptoms. Finally, for society, the economic burden related to loss of work force and medical expenses, can be reduced by a better understanding of fatigue in HD patients leading to more effective treatment options.

\section{Implementation and innovation}

Fatigue is often regarded by healthcare professionals as an inherent symptom of endstage chronic kidney disease and the HD treatment burden. ${ }^{9}$ Therefore, this disabling phenomenon with potentially serious consequences for patients and society is underrecognised by healthcare professionals and poorly assessed in trials and clinical 
practice. Recently however, the Standardized Outcomes in Nephrology (SONG) initiative identified fatigue as one of the core outcomes to be measured in future trials in HD patients. ${ }^{10}$ Regular screening and assessment of fatigue should be promoted and become part of standard clinical practice in HD patients. This may be facilitated by the development of a universally accepted patient-reported outcome measure (PROM) instrument for fatigue in HD patients. This thesis showed that the ESM incorporated in an mHealth device may be a perfect measurement instrument for a fatigue PROM in HD patients. Indeed, an mHealth ESM-based fatigue measurement instrument can be easily implemented in HD patients' daily life, allowing to identify diurnal changes in fatigue and related psychological, social and contextual factors. In this way, both HD patients and health care professionals may gain insight in fatigue symptom development and contributing factors in patients' daily life. When integrating an mHealth ESM-based fatigue measurement instrument in routine practice, it offers the opportunity to immediately discuss relevant information with patients and may identify targets for personalized interventions. Moreover, patients may feel engaged in the discussion and management of their own health and wellbeing, which may lead to a greater satisfaction of patients with their care.

\section{Dissemination of knowledge}

In order to share our research findings with other researchers, we published the results in several international scientific journals. We also presented our results at several international scientific congresses. For instance, at the Kidney Week congress of the American Society of Nephrology in 2019 and 2020, and at the $56^{\text {th }}$ and $57^{\text {th }}$ congress of the European-Renal-Association (ERA) European-Dialysis-and-TransplantAssociation (EDTA) in 2019 and 2020, respectively. Nationally, we informed clinicians about our findings on the $61^{\text {st }}$ national congress of the Italian Society of Nephrology 2020. 


\section{References}

1. Mathers CD, Loncar D. Projections of global mortality and burden of disease from 2002 to 2030. PLoS medicine. 2006;3(11):e442.

2. United States Renal Data System. USRDS annual data report: Epidemiology of kidney disease in the United States. 2018; https://www.usrds.org/2018/view/Default.aspx.

3. Heaf J. Current trends in European renal epidemiology. Clin Kidney J. 2017;10(2):149-153.

4. Kramer A, Pippias M, Noordzij M, et al. The European Renal Association - European Dialysis and Transplant Association (ERA-EDTA) Registry Annual Report 2015: a summary. Clin Kidney J. 2018; 11(1):108-122.

5. Bossola M, Vulpio C, Tazza L. Fatigue in chronic dialysis patients. Seminars in dialysis. 2011;24(5): 550555.

6. Urquhart-Secord R, Craig JC, Hemmelgarn B, et al. Patient and Caregiver Priorities for Outcomes in Hemodialysis: An International Nominal Group Technique Study. American journal of kidney diseases : the official journal of the National Kidney Foundation. 2016;68(3):444-454.

7. Weisbord SD, Fried LF, Arnold RM, et al. Prevalence, severity, and importance of physical and emotional symptoms in chronic hemodialysis patients. Journal of the American Society of Nephrology: JASN. 2005;16(8):2487-2494.

8. Jacobson J, Ju A, Baumgart A, et al. Patient Perspectives on the Meaning and Impact of Fatigue in Hemodialysis: A Systematic Review and Thematic Analysis of Qualitative Studies. American journal of kidney diseases : the official journal of the National Kidney Foundation. 2019;74(2):179-192.

9. Picariello F, Moss-Morris R, Macdougall IC, et al. Cognitive-behavioural therapy (CBT) for renal fatigue (BReF): a feasibility randomised-controlled trial of $C B T$ for the management of fatigue in haemodialysis (HD) patients. BMJ open. 2018;8(3):e020842.

10. Tong A, Manns B, Hemmelgarn B, et al. Establishing Core Outcome Domains in Hemodialysis: Report of the Standardized Outcomes in Nephrology-Hemodialysis (SONG-HD) Consensus Workshop. American journal of kidney diseases. 2017;69(1):97-107. 



\section{List of publications}


Addendum 


\section{List of publications}

\section{Articles in international peer-reviewed journals}

Brys ADH, Stifft F, van Heugten CM, Bossola M, Gambaro G, Lenaert B. mHealth based Experience Sampling Method to identify fatigue in the context of daily life in haemodialysis patients. Clin Kidney J. 2021;14 (1):245-54.

Brys ADH, Di Stasio E, Lenaert B, Picca A, Calvani R, Marzetti E, Gambaro G, Bossola M. Peridialytic serum cytokine levels and their relationship with postdialysis fatigue and recovery in patients on chronic haemodialysis. Cytokine. 2020;135:155223.

Brys ADH, Bossola M, Lenaert B, Biamonte F, Gambaro G, Di Stasio E. Daily physical activity in patients on chronic haemodialysis and its relation to fatigue and depressive symptoms. Int Urol Nephrol. 2020;52(10):1959-1967.

Brys ADH, Stifft F, Van Heugten CM, Bossola M, Gambaro G, Lenaert B. Unraveling fatigue in hemodialysis patients: comparing retrospective reports to real-time assessments with an mHealth Experienced Sampling Method. J Pain Symptom Manage. 2020;60(6):1100-1108.e2.

Brys $\mathrm{ADH}^{*}$, Di Stasio $\mathrm{E}^{*}$, Lenaert B, Sanguinetti M, Picca A, Calvani R, et al. Serum interleukin- 6 and endotoxin levels and their relationship with fatigue and depressive symptoms in patients on chronic haemodialysis. Cytokine. 2020;125:154823. ( ${ }^{*}$ shared first authorship)

Brys ADH, Lenaert B, Van Heugten CM, Gambaro G, Bossola M. Exploring the diurnal course of fatigue in patients on hemodialysis treatment and its relation with depressive symptoms and classical conditioning. J Pain Symptom Manage. 2019;57(5):890-8.e4.

Brys ADH, Vermeersch S, Forsyth R, Velkeniers B, Bravenboer B. Central diabetes insipidus: beware of Langerhans cell histiocytosis! The Netherlands journal of medicine. 2018;76(10):445-9. 
Brys A, Wijers A, de Vries M, Bouwman N, Borghans R. An unrecognized cause of dyspnoea. Nederlands tijdschrift voor geneeskunde. 2017;161:D1135.

\section{Abstracts presented at international conferences}

Brys ADH, Di stasio E , Lenaert B, Picca A, Calvani R, Marzetti E, Gambaro G, Bossola $M$. Peridialytic serum cytokine levels and their relationship with postdialysis fatigue and recovery in patients on chronic hemodialysis. Kidney Week congress of the American Society of Nephrology - Denver, U.S.A. 22-25 October 2020. Poster presentation.

Brys ADH , Bossola M, Gambaro G, Lenaert B. Merits of using mHealth Experience Sampling Methodology to assess fatigue in chronic hemodialysis patients. Kidney Week congress of the American Society of Nephrology - Denver, U.S.A. 22-25 October 2020. Poster presentation.

Brys ADH, Stifft F, Van Heugten CM, Bossola M, Gambaro G, Lenaert B. Unravelling fatigue in haemodialysis patients: a plea for the use of mobile health experience sampling methodology. 57th Congress of the European-Renal-Association (ERA)European-Dialysis-and-Transplant-Association (EDTA) - Milan, Italy. 6-9 June 2020. Poster presentation.

Brys ADH, Bossola M, Lenaert B, Biamonte F, Gambaro G, Di stasio E. Physical activity in patients on hemodialysis and its relation to fatigue and depression. Annual Kidney Week congress of the American Society of Nephrology - Washington DC, U.S.A. 7-10 November 2019. Poster presentation.

Brys ADH, Lenaert B, Van Heugten CM, Gambaro G, Bossola M. Diurnal course of fatigue in patients on haemodialysis treatment is related to depressive symptoms. 56th Congress of the European-Renal-Association (ERA)-European-Dialysis-andTransplant-Association (EDTA) - Budapest, Hungary. 13-16 June 2019. Poster presentation. 


\section{Lecture at national congress}

Brys ADH. Fatigue in haemodialysis patients: a biopsychosocial model. 61st National congress of the Italian society of Nephrology - Riva del Garda, Italy. 7-10 October 2020. 

Words of gratitude 
Addendum 


\section{Words of gratitude}

"Alone we can do so little, together we can do so much."

Helen Keller

First of all, I want to thank all the patients who participated in our studies for their time and effort. Without them, this thesis would not have been possible.

Second, I want to thank my supervisors for the opportunities they gave me, their trust, support and supervision. Professor Dr. G. Gambaro, caro professore, grazie mille per le opportunità e la fiducia sin dall'inizio. Professor C. van Heugten, beste Caroline, bedankt om mee in dit project te stappen en er een internationale dimensie aan te geven. Dr. M. Bossola and dr. B. Lenaert, caro Maurizio \& beste Bert, 'coincidence is a wonderful thing, but sometimes we are meant to run into certain people'. This certainly applies to both of you. I would never have gotten so far without the two of you. Thank you for sharing your ideas and knowledge. Thank you for the pleasant and efficient cooperation, your patience and trust, your enthusiasm and support. This is not a goodbye but a see you soon!

Thank you to the members of the assessment committees for their time and effort to evaluate this thesis.

I would like to thank all co-authors whom I had the pleasure to work, especially Dr. E. Di Stasio and dr. F. Biamonte. Caro Enrico e caro Filippo, i lupi della Sila, grazie per la collaborazione ma soprattutto per l'amicizia. Nonostante la distanza, sono convinta che rimarremo in contatto e che ci rivedremo!

Thank you to all physicians, nurses, research assistants and supporting staff of the dialysis unit of both the Gemelli Hospital (Rome) and Zuyderland Medical Centre (Sittard-Geleen and Heerlen) who in one way or another facilitated our research studies. Dr.ssa De Fazio e Dr.ssa Giungi, Silvia e Stefania, è stato un piacere conoscervi! Dr. F. Stifft, beste Frank, een welgemeende merci dat ik bij jullie team 
terecht kon voor mijn studies in Nederland. Het voelde als een 'thuismatch' en het is altijd prettig samenwerken met jullie!

Thank you to Liesbeth Mariën and Tiny Wouters for taking care of this thesis' cover and lay-out, respectively. Excellent job!

I would like to thank my (former) supervisors of internal medicine for supporting my idea to do a PhD abroad. Dr. Looij, bedankt voor dat kleine duwtje in de rug om mijn dromen na te jagen. Professor Dr. Koopmans, uw reactie op mijn verzoek om drie jaar promotieonderzoek in Rome te doen ("Prachtig, dat moet je doen!"), zal ik nooit vergeten. Uw enthousiasme werkt aanstekelijk!

I would also like to thank all the physicians from Zuyderland Medical Centre and UZ Brussels who contributed to my medical training so far and taught me that good clinical practice and research go hand in hand. I learned so much from your supervision and you remain an inspiration for my future medical career.

Maaike and Fabiënne, chance made us colleagues but fun made us friends. Thanks for sharing good and bad times! Thanks for your ideas, help, support, phone calls and visits! May there be many more beautiful moments to share!

Thank you to all my Roman friends - Suzanne, Anne, Liza, Charlotte, Jessica, Henriëtte, Martine, Lucia, Elena, Anne Bot and Marie - for all good laughter and distractions from sciences! I have so many great memories of our shared and special moments in Rome and other Italian cities. Spero di vedervi presto - \# andràtuttobene!

Stefania Catalani, my running race buddy. Grazie per la tua compagnia. Un giorno la Roma-Ostia e la maratona di Roma saranno nostre, ne sono certa! Grazie per le belle cene passate a casa vostra. Rimarrano sempre nei miei riccordi.

My dearest friends at home - Charlotte Vdf, Lynn, Tine B, Annelies, Tine T, Charlotte DH, Astrid, Henrike, Ellen, Evelyne, Hazel en Karlien. Bedankt voor jullie mental support van op afstand en voor jullie bezoekjes in Rome. Prachtige herinneringen die ik koester! 
I would like to thank my Italian host family, Renato and Gilda and their family. Being part of your family has been a unique experience that changed me and charted a path I never expected. Italy became my second home country. Grazie mille per avermi ospitata 15 anni fa a casa vostra e avermi trasformata in una cosmopolita!

I want to thank my sister and cousin. Melissa, bol, having a sister is like having a best friend you can't get rid of. You've always been there and, whatever I do, you'll always be there. Thank you for your support. You and Aäron are doing so well together! Chloë, griet, thank you for being such a funny cousin and sending me literally tons of letters with your thoughtful advice in case I needed new inspiration, some motivation, a new moto, ... and so on!

Can't wait to go on holiday again with you guys, - alleeeejjinaaa!

Ohana means family, and family means nobody gets left behind.

Last but not least, I want to thank my parents, without whom I would not be where and who I am today. Liefste mama en papa, onze eigen Philemon en Baucis, dankzij jullie konden wij zorgeloos opgroeien in een warm nest en nog steeds komen wij op de eerste plaats. Jullie onvoorwaardelijke liefde en continue steun zijn van onschatbare waarde en mijn allergrootste voorbeeld. 'Alone we can do so little, together we can do so much', heb ik bij jullie geleerd. Bedankt om er altijd te zijn en me telkens weer te volgen in mijn ideeën en dromen. 

Curriculum Vitae 
Addendum 


\section{Curriculum Vitae}

Astrid Brys was born on March $27^{\text {th }} 1988$, in Lier (Belgium). In 2006, she graduated in Greek-Latin at Sint-Ursulalyceum in Lier. From September 2006 until June 2007, she studied and lived abroad in San Benedetto del Tronto (Italy) in the context of the World Education Program (WEP). Thereafter, in September 2007, she started medical school at the Catholic University of Leuven (KU Leuven) in Belgium and graduated magna cum laude in 2014. Two months later, she moved to the Netherlands and started her residency in Internal Medicine at Maastricht University Medical Center under the supervision of prof. dr. C. Stehouwer/ prof. dr. R. Koopmans. From September 2014 until august 2017, she worked as a resident in Internal Medicine at Zuyderland Medical Centre in Sittard-Geleen under the supervision of successively Dr. B-J Looij and Dr. J. Buys. Next, she moved to Brussels (Belgium) and spent six months of her residency abroad at the University Hospital Brussels (UZ Brussels) under the supervision of prof dr. B. Velkeniers/ prof. dr. B. Bravenboer. In 2018, she temporary interrupted her residency and moved to Italy, where she started a double PhD at the Catholic University of the Sacred Heart (UCSC, Rome) in conjunction with Maastricht University (UM, Maastricht) under the supervision of prof. dr. G. Gambaro and Dr. M. Bossola, and prof. dr. C. van Heugten and dr. B. Lenaert, respectively. The focus of her research was on fatigue in patients on chronic haemodialysis treatment. She conducted studies in both Italy and the Netherlands. The scientific results of this period are presented in this thesis. Astrid's PhD was financed by the UCSC, headquartered in Milan. In January 2021, she resumed her residency in Internal Medicine (geriatrics) at Maastricht University Medical Center. 
Addendum 